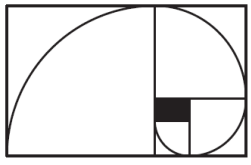

\title{
Current fluctuations for stochastic particle systems with drift in one spatial dimension
}

\author{
Timo Seppäläinen
}

\begin{abstract}
This review article discusses limit distributions and variance bounds for particle current in several dynamical stochastic systems of particles on the one-dimensional integer lattice: independent particles, independent particles in a random environment, the random average process, the asymmetric simple exclusion process, and a class of totally asymmetric zero range processes. The first three models possess linear macroscopic flux functions and lie in the Edwards-Wilkinson universality class with scaling exponent $1 / 4$ for current fluctuations. For these we prove Gaussian limits for the current process. The latter two systems belong to the Kardar-Parisi-Zhang class. For these we prove the scaling exponent $1 / 3$ in the form of upper and lower variance bounds.
\end{abstract}

2000 Mathematics Subject Classification: 60K35, 60F05, 60K37. 



\section{Acknowledgments}

This article is based on lecture notes for a minicourse given at the 13th Brazilian School of Probability, August 2-8, 2009, at Maresias, São Paulo, and again at the University of Helsinki, Finland, on August 18-20, 2009. The author thanks the organizers and the audiences of these two occasions. The material comes from collaborations with Márton Balázs, Mathew Joseph, Júlia Komjáthy, Rohini Kumar, Jon Peterson and Firas Rassoul-Agha. The author is grateful for financial support from the National Science Foundation through grant DMS-0701091 and from the Wisconsin Alumni Research Foundation. 



\section{Contents}

$\begin{array}{lll}1 & \text { Introduction } & 7\end{array}$

2 Independent particles executing classical random walks 10

2.1 Model and results . . . . . . . . . . . . . . . . . . . 10

2.2 Sketch of proof . . . . . . . . . . . . . . . . 14

3 Independent particles in a random environment $\quad 20$

3.1 Model and results . . . . . . . . . . . . . . . . . . 20

3.2 Sketch of the proof for the quenched mean of the current . 25

4 Random average process $\quad 28$

4.1 Model and results . . . . . . . . . . . . . . . . . . . . . . . . 28

4.2 Steps of the proof . . . . . . . . . . . . . . . . 30

5 Asymmetric simple exclusion process 41

5.1 Basic properties . . . . . . . . . . . . . . . . 41

5.2 Results.......................... . . . . 44

5.3 Proofs for the identities . . . . . . . . . . . . . . 46

5.4 A coupling and a random walk bound . . . . . . . . . . . . 49

5.5 Proof of the upper bound for second class particle moments 54

5.6 Proof of the lower bound for second class particle moments 61

6 Zero range process $\quad \mathbf{6 6}$

6.1 Model and results . . . . . . . . . . . . . . . . . . . . . 66

6.2 Variance identity . . . . . . . . . . . . . . . . . . . 70

6.3 Coupling for the zero range process . . . . . . . . . . . 71

$\begin{array}{ll}\text { Bibliography } & 77\end{array}$ 



\section{Chapter 1}

\section{Introduction}

This review article investigates the process of particle current in several conservative stochastic systems of particles that live on the one-dimensional integer lattice. In conservative systems particles are neither created nor destroyed. On the macrosopic, deterministic scale the particle density $\rho(t, x)$ of such systems is governed by a partial differential equation of scalar conservation law type: $\rho_{t}+H(\rho)_{x}=0$. The results covered in this article concern the fluctuation behavior of the current, and include some precise limit results and some coarser order-of-magnitude bounds. Some background on advanced probability theory is assumed. For readers with little probability background we can also suggest the review article [Sep08] where an attempt was made to explain some of this same material for a reader with background in analysis rather than probability.

The particle processes studied fall into two categories. We can define these two categories by the slope of the flux function $H$ : (i) processes with a linear flux and (ii) those with a strictly concave flux. By definition, the flux $H(\rho)$ is the mean rate at which particle mass moves past a fixed point in space when the system is stationary in both space and time and has overall density $\rho$ of particles. The processes we study are asymmetric or driven in the sense that the particles have a drift, that is, a preferred average direction. This assumption is not necessary for the results for systems with linear flux, but for those with nonlinear flux it is crucial.

In the statistical physics terminology of surface growth, we can also label these two classes as (i) the EW (Edwards-Wilkinson) and (ii) the KPZ (Kardar-Parisi-Zhang) universality classes [BS]. Surface growth may seem at first a separate topic from particle systems. But in one dimension conservative particle systems can be equivalently formulated as interface models. The connection goes by way of regarding the particle occupation numbers $\eta_{i}$ as increments, or discrete gradients, of the interface height function: $h_{i}=\eta_{i}-\eta_{i-1}$. Then any movement of particles in the conserva- 
tive particle system can be equivalently described as deposition or removal of particles from the growing surface. In particular, the current process then maps directly into the height function.

In the EW class limiting current/height fluctuations are described by the linear stochastic heat equation $Z_{t}=\nu Z_{x x}+\dot{W}$ where subscripts are partial derivatives and $\dot{W}$ represents space-time white noise. In the microscopic model the order of magnitude of current fluctuations is $n^{1 / 4}$ in terms of a scaling parameter $n(n \nearrow \infty)$ that gives both the space and time scale. The results we give describe Gaussian limit distributions of the current process. The examples of systems with linear flux we cover are independent random walks, independent random walks in a random environment (RWRE), and the random average process.

In the RWRE case we in particular wish to understand how the environment affects the fluctuations. We find a two-level fluctuation picture. On the diffusive $n^{1 / 2}$ scale the quenched mean of the current converges to a Brownian motion. Around the quenched mean, on the $n^{1 / 4}$ scale, the current converges to the Gaussian processes that arose in the case of independent classical random walks, but with an additional Brownian random spatial shift.

The systems with concave flux that we discuss are the asymmetric simple exclusion process (ASEP) and a class of zero range processes. In this case the assumption of asymmetry is necessary. We consider only stationary systems (or small perturbations thereof), and instead of distributional limits we give only bounds on the variance of the current and on the moments of a second class particle. The current fluctuations are now of order $t^{1 / 3}$. This order of magnitude goes together with superdiffusivity of the second class particle whose fluctuations are of order $t^{2 / 3}$. Here $t$ is the time parameter of the process. A separate scaling parameter is not needed since we have no process level result. These systems are in the KPZ class.

We cannot cover complete proofs for all results to keep this article at a reasonable length. The most important result, namely the moment bounds for the second class particle in ASEP, is proved in full detail, assuming some basic facts about ASEP.

In some sense interface height in the KPZ class should be described by the KPZ equation

$$
h_{t}=\nu h_{x x}-\lambda\left(h_{x}\right)^{2}+\dot{W} .
$$

However, giving mathematical meaning to this equation has been done only indirectly. A formal Hopf-Cole transformation $Z=\exp \left(-\lambda \nu^{-1} h\right)$ converts (1.1) into a stochastic heat equation with multiplicative noise: $Z_{t}=\nu Z_{x x}+\lambda \nu^{-1} Z \dot{W}$. The solution of this latter equation is well-defined, and can furthermore be obtained as a limit of an appropriately scaled height function of a weakly asymmetric simple exclusion process [BG97]. Via this link the scaling exponents have recently been verified for the Hopf- 
Cole solution of the KPZ equation [BQS].

The most glaring omission of this article is that we do not treat the Tracy-Widom fluctuations of KPZ systems such as TASEP (totally asymmetric simple exclusion process), ASEP or the PNG (polynuclear growth) model. In the ASEP section we only state briefly the Ferrari-Spohn theorem [FS06] on the limit distribution of the current across a characteristic in stationary TASEP. This area is advancing rapidly and requires a review article of its own if any degree of detail is to be covered. Tracy and Widom have recently written a series of papers where the fluctuation limits originally proved for TASEP by Johansson [Joh00] have been extended to ASEP [TW08a, TW08b, TW09a, TW09b, TW10]. Another line of recent work extends the TASEP limits to more general space-time points and initial distributions $[\mathrm{BFP}, \mathrm{CFP}]$.

A notational convention. $\mathbb{Z}_{+}=\{0,1,2,3, \ldots\}$ and $\mathbb{N}=\{1,2,3, \ldots\}$. 


\section{Chapter 2}

\section{Independent particles executing classical random walks}

\subsection{Model and results}

Fix a probability distribution $p(x)$ on $\mathbb{Z}$. Let us assume for simplicity that $p(x)$ has finite range, that is,

$$
\text { the set }\{x \in \mathbb{Z}: p(x)>0\} \text { is finite. }
$$

For each site $x \in \mathbb{Z}$ and index $k \in \mathbb{N}$ let $X^{x, k}$ be a discrete-time random walk with initial point $X_{0}^{x, k}=x$ and transition probability

$$
P\left(X_{s+1}^{x, k}=y \mid X_{s}^{x, k}=z\right)=p(y-z)
$$

for times $s \in \mathbb{Z}_{+}$and space points $z, y \in \mathbb{Z}$. The walks $\left\{X^{x, k}: x \in \mathbb{Z}, k \geq\right.$ $1\}$ are independent of each other. Let

$$
v=\sum_{x \in \mathbb{Z}} x p(x) \quad \text { and } \quad \sigma_{1}^{2}=\sum_{x \in \mathbb{Z}}(x-v)^{2} p(x)
$$

be the mean speed and variance of the walks.

At time 0 we start a random number $\eta_{x}(0)$ of particles at site $x$. The assumption is that the initial occupation variables $\eta(0)=\left\{\eta_{x}(0): x \in \mathbb{Z}\right\}$ are i.i.d. (independent and identically distributed) with finite mean and variance

$$
\mu_{0}=E\left[\eta_{x}(0)\right] \quad \text { and } \quad \sigma_{0}^{2}=\operatorname{Var}\left[\eta_{x}(0)\right]
$$


Furthermore, the variables $\left\{\eta_{x}(0)\right\}$ and the walks $\left\{X_{.}^{x, k}\right\}$ are independent of each other. If the locations of individual particles are not of interest but only the overall particle distribution, the particle configuration at time $s \in \mathbb{N}$ is described by the occupation variables $\eta(s)=\left\{\eta_{x}(s): x \in \mathbb{Z}\right\}$ defined as

$$
\eta_{x}(s)=\sum_{y \in \mathbb{Z}} \sum_{k=1}^{\eta_{0}(y)} \mathbf{1}\left\{X_{s}^{y, k}=x\right\} .
$$

A basic fact is that if the initial occupation variables are i.i.d. Poisson with common mean $\mu$, then so are the occupation $\left\{\eta_{x}(s): x \in \mathbb{Z}\right\}$ at any fixed time $s \in \mathbb{Z}_{+}$. This invariance follows easily from basic properties of Poisson distributions: the counts of particles that move from $x$ to $y$ are independent Poisson variables across all pairs $(x, y)$.

Let $E^{\mu}$ denote expectation under the stationary process with mean $\mu$ occupations. The flux function is the expected rate of flow in the stationary process:

$$
\begin{aligned}
H(\mu) & =E^{\mu}[\text { net number of particles that jump across edge }(0,1) \\
& \text { left to right in one time step] } \\
& =E^{\mu}\left[\sum_{x \leq 0} \sum_{k=1}^{\eta_{0}(x)} \mathbf{1}\left\{X_{1}^{x, k} \geq 1\right\}-\sum_{x>0} \sum_{k=1}^{\eta_{0}(x)} \mathbf{1}\left\{X_{1}^{x, k} \leq 0\right\}\right] \\
& =v \mu .
\end{aligned}
$$

The linear flux puts this system in the class where we expect $n^{1 / 4}$ magnitude current fluctuations. Next we define the current process and the Gaussian process that describes the limit.

Let $n \in \mathbb{N}$ denote a scaling parameter that eventually goes to $\infty$. For (macroscopic) times $t \in \mathbb{R}_{+}$and a spatial variable $r \in \mathbb{R}$, let

$$
Y_{n}(t, r)=Y_{n, 1}(t, r)-Y_{n, 2}(t, r)
$$

with

$$
Y_{n, 1}(t, r)=\sum_{x \leq 0} \sum_{k=1}^{\eta_{x}(0)} \mathbf{1}\left\{X_{\lfloor n t\rfloor}^{x, k}>\lfloor n t v\rfloor+r \sqrt{n}\right\}
$$

and

$$
Y_{n, 2}(t, r)=\sum_{x>0} \sum_{k=1}^{\eta_{x}(0)} \mathbf{1}\left\{X_{\lfloor n t\rfloor}^{x, k} \leq\lfloor n t v\rfloor+r \sqrt{n}\right\} .
$$

The variable $Y_{n}(t, r)$ represents the net left-to-right current of particles seen by an observer who starts at the origin and reaches point $\lfloor n t v\rfloor+$ $\lfloor r \sqrt{n}\rfloor$ in time $\lfloor n t\rfloor$. Its mean is

$$
E Y_{n}(t, r)=\mu_{0} E\left(X_{\lfloor n t\rfloor}-\lfloor n t v\rfloor-\lfloor r \sqrt{n}\rfloor\right)=-\mu_{0} r \sqrt{n}+O(1) .
$$


Define the centered and appropriately scaled process by

$$
\bar{Y}_{n}(t, r)=n^{-1 / 4}\left(Y_{n}(t, r)-E Y_{n}(t, r)\right) .
$$

The goal is to prove a limit for the joint distributions of these random variables. We will not tackle process-level convergence. But let us point out that there is a natural path space $D_{2}$ of functions of two parameters $(t, r)$ that contains the paths of the processes $Y_{n}$. Elements of $D_{2}$ are continuous from above with limits from below in a suitable way, and there is a metric that generalizes the standard Skorohod topology of the usual $D$-space of cadlag paths. (See [BW71, Kum08].)

Let

$$
\varphi_{\nu^{2}}(x)=\frac{1}{\sqrt{2 \pi \nu^{2}}} \exp \left(-\frac{x^{2}}{2 \nu^{2}}\right) \quad \text { and } \quad \Phi_{\nu^{2}}(x)=\int_{-\infty}^{x} \varphi_{\nu^{2}}(y) d y
$$

denote the centered Gaussian density with variance $\nu^{2}$ and its distribution function. Let $W$ be a two-parameter Brownian motion on $\mathbb{R}_{+} \times \mathbb{R}$ and $B$ a two-sided one-parameter Browian motion on $\mathbb{R}$. $W$ and $B$ are independent. Define the process $Z$ by

$$
\begin{gathered}
Z(t, r)=\sqrt{\mu_{0}} \iint_{[0, t] \times \mathbb{R}} \varphi_{\sigma_{1}^{2}(t-s)}(r-x) d W(s, x) \\
+\sigma_{0} \int_{\mathbb{R}} \varphi_{\sigma_{1}^{2} t}(r-x) B(x) d x
\end{gathered}
$$

$\left\{Z(t, r): t \in \mathbb{R}_{+}, r \in \mathbb{R}\right\}$ is a mean zero Gaussian process. Its covariance can be expressed as follows: with

$$
\Psi_{\nu^{2}}(x)=\nu^{2} \varphi_{\nu^{2}}(x)-x\left(1-\Phi_{\nu^{2}}(x)\right)
$$

define

$$
\Gamma_{1}((s, q),(t, r))=\Psi_{\sigma_{1}^{2}(t+s)}(r-q)-\Psi_{\sigma_{1}^{2}|t-s|}(r-q)
$$

and

$$
\Gamma_{2}((s, q),(t, r))=\Psi_{\sigma_{1}^{2} s}(-q)+\Psi_{\sigma_{1}^{2} t}(r)-\Psi_{\sigma_{1}^{2}(t+s)}(r-q) .
$$

Then

$$
\mathbf{E}[Z(s, q) Z(t, r)]=\mu_{0} \Gamma_{1}((s, q),(t, r))+\sigma_{0}^{2} \Gamma_{2}((s, q),(t, r)) .
$$

Remark 2.1. Some comments on Gaussian processes defined above. Comparison of (2.8) and (2.12) shows that $\Gamma_{1}$ is the covariance of the dynamical fluctuations represented by the space-time white noise $d W$-integral, and $\Gamma_{2}$ 
is the covariance of the contribution of the initial fluctuations represented by the Brownian motion $\sigma_{0} B(\cdot)$.

$\Gamma_{1}$ has this alternative formula:

$$
\Gamma_{1}((s, q),(t, r))=\frac{1}{2} \int_{\sigma_{1}^{2}|t-s|}^{\sigma_{1}^{2}(t+s)} \frac{1}{\sqrt{2 \pi v}} \exp \left\{\frac{1}{2 v}(r-q)^{2}\right\} d v .
$$

To verify that the process

$$
\zeta(t, r)=\iint_{[0, t] \times \mathbb{R}} \varphi_{\sigma_{1}^{2}(t-s)}(r-x) d W(s, x)
$$

has covariance

$$
\mathbf{E} \zeta(s, q) \zeta(t, r)=\Gamma_{1}((s, q),(t, r))
$$

is straightforward from the general property that for $f, g \in L^{2}\left(\mathbb{R}^{d}\right)$ the white-noise integrals $\int f d W$ and $\int g d W$ on $\mathbb{R}^{d}$ are by definition mean zero Gaussian random variables that satisfy

$$
\mathbf{E}\left[\left(\int f d W\right)\left(\int g d W\right)\right]=\int_{\mathbb{R}^{d}} f(x) g(x) d x .
$$

To say that $B(x)$ is a (standard) two-sided Brownian motion means that we take two independent standard Brownian motions $B_{1}$ and $B_{2}$ and set

$$
B(x)= \begin{cases}B_{1}(x), & x \geq 0 \\ B_{2}(-x), & x<0\end{cases}
$$

To show that the process

$$
\xi(t, r)=\int_{\mathbb{R}} \varphi_{\sigma_{1}^{2} t}(r-x) B(x) d x
$$

is a mean-zero Gaussian process with covariance

$$
\mathbf{E} \xi(s, q) \xi(t, r)=\Gamma_{2}((s, q),(t, r))
$$

this formula is useful: if $f, g$ are absolutely continuous functions on $\mathbb{R}_{+}$ such that $x f(x) \rightarrow 0$ and $x g(x) \rightarrow 0$ as $x \rightarrow \infty$, then

$$
\iint_{\mathbb{R}_{+}^{2}} f^{\prime}(x) g^{\prime}(y)(x \wedge y) d x d y=\int_{0}^{\infty} f(x) g(x) d x .
$$

It is also the case that the process $Z(t, r)$ is the unique weak solution of the following initial value problem for a linear stochastic heat equation on $\mathbb{R}_{+} \times \mathbb{R}:$

$$
Z_{t}=\frac{\sigma_{1}^{2}}{2} Z_{r r}+\sqrt{\mu_{0}} \dot{W}, \quad Z(0, r)=\sigma_{0} B(r) .
$$


(Above, subscript means partial derivative.) A weak solution of this equation is defined by the requirement

$$
\begin{aligned}
& \int_{\mathbb{R}} \phi(r) Z(t, r) d r-\sigma_{0} \int_{\mathbb{R}} \phi(r) B(r) d r \\
& =\frac{\sigma_{1}^{2}}{2} \iint_{[0, t] \times \mathbb{R}} \phi^{\prime \prime}(r) Z(s, r) d r d s+\sqrt{\mu}_{0} \iint_{[0, t] \times \mathbb{R}} \phi(r) d W(s, r)
\end{aligned}
$$

for all $\phi \in C_{c}^{\infty}(\mathbb{R})$ (compactly supported, infinitely differentiable). See the lecture notes of Walsh [Wal86].

We can now state the main result.

Theorem 2.2. Assume the initial occupation variables are i.i.d. with finite mean and variance as in (2.2). Then as $n \rightarrow \infty$, the finite-dimensional distributions of the process $\left\{\bar{Y}_{n}(t, r):(t, r) \in \mathbb{R}_{+} \times \mathbb{R}\right\}$ converge weakly to the finite-dimensional distributions of the mean zero Gaussian process $\left\{Z(t, r):(t, r) \in \mathbb{R}_{+} \times \mathbb{R}\right\}$.

The statement means that for any space-time points $\left(t_{1}, r_{1}\right), \ldots,\left(t_{k}, r_{k}\right)$, this weak convergence of $\mathbb{R}^{k}$-valued random vectors holds:

$$
\left(\bar{Y}_{n}\left(t_{1}, r_{1}\right), \ldots, \bar{Y}_{n}\left(t_{k}, r_{k}\right)\right) \stackrel{\mathcal{D}}{\longrightarrow}\left(Z\left(t_{1}, r_{1}\right), \ldots, Z\left(t_{k}, r_{k}\right)\right) .
$$

Under additional moment assumptions process level convergence in the space $D_{2}$ can be proved (see [Kum08]). We state a corollary for the special case of the stationary occupation process $\eta(t)$. Its proof comes from simplifying expression (2.12) for the covariance.

Corollary 2.1. Suppose the process is stationary so that $\left\{\eta_{x}(t): x \in \mathbb{Z}\right\}$ are i.i.d. Poisson with mean $\mu_{0}$ for each fixed $t$. Then at $r=0$ the limit process $Z$ has covariance

$$
\mathbf{E} Z(s, 0) Z(t, 0)=\frac{\mu_{0} \sigma_{1}}{\sqrt{2 \pi}}(\sqrt{s}+\sqrt{t}-\sqrt{|t-s|}) .
$$

In other words, process $Z(\cdot, 0)$ is fractional Brownian motion with Hurst parameter $1 / 4$.

\subsection{Sketch of proof}

We turn to discuss the proof of Theorem 2.2. Independent walks allow us to compute everything in a straightforward manner. Fix some $N \in \mathbb{N}$, time points $0<t_{1}<t_{2}<\cdots<t_{N} \in \mathbb{R}_{+}$, space points $r_{1}, r_{2}, \ldots, r_{N} \in \mathbb{R}$ and an $N$-vector $\boldsymbol{\theta}=\left(\theta_{1}, \ldots, \theta_{N}\right) \in \mathbb{R}^{N}$. Form the linear combinations

$$
\bar{Y}_{n}(\boldsymbol{\theta})=\sum_{i=1}^{N} \theta_{i} \bar{Y}_{n}\left(t_{i}, r_{i}\right) \quad \text { and } \quad Z(\boldsymbol{\theta})=\sum_{i=1}^{N} \theta_{i} Z\left(t_{i}, r_{i}\right) .
$$


The goal is now to prove

\section{Proposition 2.1.}

$$
E\left[\exp \left\{i \bar{Y}_{n}(\boldsymbol{\theta})\right\}\right] \rightarrow \mathbf{E}[\exp \{i Z(\boldsymbol{\theta})\}]
$$

Since the random walks and initial occupation variables are independent, we can write $\bar{Y}_{n}(\boldsymbol{\theta})$ as a sum of independent random variables and take advantage of standard central limit theorems from the literature.

$$
\bar{Y}_{n}(\boldsymbol{\theta})=n^{-\frac{1}{4}} \sum_{i=1}^{N} \theta_{i}\left\{Y_{n}\left(t_{i}, r_{i}\right)-E Y_{n}\left(t_{i}, r_{i}\right)\right\}=W_{n}=\sum_{m=-\infty}^{\infty} u(m)
$$

with

$$
u(m)=\sum_{i=1}^{N} \theta_{i}\left(U_{m}\left(t_{i}, r_{i}\right) \mathbf{1}\{m \leq 0\}-V_{m}\left(t_{i}, r_{i}\right) \mathbf{1}\{m>0\}\right),
$$

and

$$
\begin{gathered}
U_{m}(t, r)=n^{-\frac{1}{4}} \sum_{j=1}^{\eta_{m}(0)} \mathbf{1}\left\{X_{n t}^{m, j}>\lfloor n t v\rfloor+r \sqrt{n}\right\} \\
-n^{-\frac{1}{4}} \mu_{0} P\left(X_{n t}^{m}>\lfloor n t v\rfloor+r \sqrt{n}\right), \\
V_{m}(t, r)=n^{-\frac{1}{4}} \sum_{j=1}^{\eta_{m}(0)} \mathbf{1}\left\{X_{n t}^{m, j} \leq\lfloor n t v\rfloor+r \sqrt{n}\right\} \\
-n^{-\frac{1}{4}} \mu_{0} P\left(X_{n t}^{m} \leq\lfloor n t v\rfloor+r \sqrt{n}\right) .
\end{gathered}
$$

The variables $\{u(m)\}_{m \in \mathbb{Z}}$ are independent because initial occupation variables and walks are independent.

Let $a(n) \nearrow \infty$ be a sequence that will be determined precisely in the proof. As the first step we observe that the terms $|m|>a(n) \sqrt{n}$ can be discarded from (2.16). Define

$$
W_{n}^{*}=\sum_{|m| \leq a(n) \sqrt{n}} u(m) \text {. }
$$

The lemma below is proved by calculating moments of the random walks.

Lemma 2.1. $E\left|W_{n}-W_{n}^{*}\right|^{2} \rightarrow 0$ as $n \rightarrow \infty$. 
The limit $Z(\boldsymbol{\theta})$ in our goal (2.15) has $\mathcal{N}\left(0, \sigma(\boldsymbol{\theta})^{2}\right)$ distribution with variance

$\sigma(\boldsymbol{\theta})^{2}=\sum_{1 \leq i, j \leq N} \theta_{i} \theta_{j}\left[\mu_{0} \Gamma_{1}\left(\left(t_{i}, r_{i}\right),\left(t_{j}, r_{j}\right)\right)+\sigma_{0}^{2} \Gamma_{2}\left(\left(t_{i}, r_{i}\right),\left(t_{j}, r_{j}\right)\right)\right]$.

The two $\Gamma$-terms, defined earlier in (2.10) and (2.11), have the following expressions in terms of a standard 1-dimensional Brownian motion $B_{t}$ :

$$
\begin{aligned}
\Gamma_{1}((s, q),(t, r)) & =\int_{-\infty}^{\infty}\left(\mathbf{P}\left[B_{\sigma_{1}^{2} s} \leq q-x\right] \mathbf{P}\left[B_{\sigma_{1}^{2} t}>r-x\right]\right. \\
& \left.-\mathbf{P}\left[B_{\sigma_{1}^{2} s} \leq q-x, B_{\sigma_{1}^{2} t}>r-x\right]\right) d x
\end{aligned}
$$

and

$$
\begin{gathered}
\Gamma_{2}((s, q),(t, r))=\int_{-\infty}^{0} \mathbf{P}\left[B_{\sigma_{1}^{2} s}>q-x\right] \mathbf{P}\left[B_{\sigma_{1}^{2} t}>r-x\right] d x \\
+\int_{0}^{\infty} \mathbf{P}\left[B_{\sigma_{1}^{2} s} \leq q-x\right] \mathbf{P}\left[B_{\sigma_{1}^{2} t} \leq r-x\right] d x .
\end{gathered}
$$

Remark 2.3. Turning formulas (2.21)-(2.22) into (2.10)-(2.11) involves calculus and these properties of Gaussians: $(d / d x) \Psi_{\nu^{2}}(x)=-\Phi_{\nu^{2}}(-x)$, $\Phi_{\nu^{2}}(x)=1-\Phi_{\nu^{2}}(-x)$ and

$$
\int_{-\infty}^{\infty} \Phi_{\alpha^{2}}(x) \Phi_{\nu^{2}}(r-x) d x=\int_{-\infty}^{r} \Phi_{\alpha^{2}+\nu^{2}}(x) d x .
$$

By Lemma 2.1, the desired limit (2.15) follows from showing

$$
E\left(e^{i W_{n}^{*}}\right) \rightarrow e^{-\sigma(\boldsymbol{\theta})^{2} / 2}
$$

This will be achieved by the Lindeberg-Feller theorem.

Theorem 2.4 (Lindeberg-Feller). For each $n$, suppose $\left\{X_{n, j}: 1 \leq j \leq\right.$ $J(n)\}$ are independent, mean-zero, square-integrable random variables and let $S_{n}=X_{n, 1}+\cdots+X_{n, J(n)}$. Assume that

$$
\lim _{n \rightarrow \infty} \sum_{j=1}^{J(n)} E\left(X_{n, j}^{2}\right)=\sigma^{2}
$$

and for each $\varepsilon>0$,

$$
\lim _{n \rightarrow \infty} \sum_{j=1}^{J(n)} E\left(X_{n, j}^{2} \mathbf{1}\left\{\left|X_{n, j}\right| \geq \varepsilon\right\}\right)=0 .
$$


Then as $n \rightarrow \infty, S_{n}$ converges in distribution to a $\mathcal{N}\left(0, \sigma^{2}\right)$-distributed Gaussian random variable. In terms of probabilities, the conclusion is that for all $s \in \mathbb{R}$,

$$
\lim _{n \rightarrow \infty} P\left\{S_{n} \leq s\right\}=\frac{1}{\sqrt{2 \pi \sigma^{2}}} \int_{-\infty}^{s} e^{-x^{2} / 2 \sigma^{2}} d x .
$$

In terms of characteristic functions, the conclusion is that for all $t \in \mathbb{R}$,

$$
\lim _{n \rightarrow \infty} E\left(e^{i t S_{n}}\right)=e^{-\sigma^{2} t^{2} / 2} .
$$

Now to prove (2.23) the task is to verify the conditions of the LindebergFeller theorem:

$$
\sum_{|m| \leq a(n) \sqrt{n}} E\left(u(m)^{2}\right) \rightarrow \sigma(\boldsymbol{\theta})^{2}
$$

and

$$
\sum_{|m| \leq a(n) \sqrt{n}} E\left(|u(m)|^{2} \mathbf{1}\{|u(m)| \geq \varepsilon\}\right) \rightarrow 0 .
$$

We begin with the negligibility condition (2.25). This will determine $a(n)$.

Lemma 2.2. Under assumption (2.2),

$$
\lim _{n \rightarrow \infty} \sum_{|m| \leq a(n) \sqrt{n}} E\left(|u(m)|^{2} \mathbf{1}\{|u(m)| \geq \varepsilon\}\right)=0 .
$$

Proof. Since

$$
|u(m)| \leq C n^{-1 / 4}\left(\eta_{m}(0)+\mu_{0}\right),
$$

for a different $\varepsilon_{1}>0$ and by shift-invariance,

$$
\begin{gathered}
\sum_{|m| \leq a(n) \sqrt{n}} E\left[|u(m)|^{2} \mathbf{1}\{|u(m)| \geq \varepsilon\}\right] \\
\leq C a(n) E\left[\left(\eta_{0}(0)+\mu_{0}\right)^{2} \mathbf{1}\left\{\eta_{0}(0) \geq n^{1 / 4} \varepsilon_{1}\right\}\right] .
\end{gathered}
$$

By the moment assumption (2.2) this last expression $\rightarrow 0$ for every $\varepsilon_{1}>0$ if $a(n) \nearrow \infty$ slowly enough, for example

$$
a(n)=\left(E\left[\left(\eta_{0}(0)+\mu_{0}\right)^{2} \mathbf{1}\left\{\eta_{0}(0) \geq n^{1 / 8}\right\}\right]\right)^{-1 / 2}
$$

We turn to checking (2.24).

$$
\begin{aligned}
& \sum_{|m| \leq a(n) \sqrt{n}} E\left[u(m)^{2}\right] \\
& =\sum_{1 \leq i, j \leq N} \theta_{i} \theta_{j} \sum_{|m| \leq a(n) \sqrt{n}}\left[\mathbf{1}_{\{m \leq 0\}} E\left(U_{m}\left(t_{i}, r_{i}\right) U_{m}\left(t_{j}, r_{j}\right)\right)\right. \\
& \left.+\mathbf{1}_{\{m>0\}} E\left(V_{m}\left(t_{i}, r_{i}\right) V_{m}\left(t_{j}, r_{j}\right)\right)\right] .
\end{aligned}
$$


To the expectations we apply this formula for the covariance of two random sums: with $\left\{Z_{i}\right\}$ i.i.d. and independent of a random $K \in \mathbb{Z}_{+}$,

$$
\begin{aligned}
& \operatorname{Cov}\left(\sum_{i=1}^{K} f\left(Z_{i}\right), \sum_{j=1}^{K} g\left(Z_{j}\right)\right) \\
& =E K \operatorname{Cov}(f(Z), g(Z))+\operatorname{Var}(K) E f(Z) E g(Z) .
\end{aligned}
$$

For the first expectation on the right in (2.27):

$$
\begin{aligned}
& E\left(U_{m}(s, q) U_{m}(t, r)\right) \\
& =n^{-1 / 2} \operatorname{Cov}\left(\sum_{j=1}^{\eta_{m}(0)} \mathbf{1}\left\{X_{n s}^{m, j}>\lfloor n s v\rfloor+q \sqrt{n}\right\},\right. \\
& \left.\sum_{j=1}^{\eta_{m}(0)} \mathbf{1}\left\{X_{n t}^{m, j}>\lfloor n t v\rfloor+r \sqrt{n}\right\}\right) \\
& =n^{-1 / 2} \mu_{0}\left[P\left(X_{n s}^{m}>\lfloor n s v\rfloor+q \sqrt{n}, X_{n t}^{m}>\lfloor n t v\rfloor+r \sqrt{n}\right)\right. \\
& \left.\quad-P\left(X_{n s}^{m}>\lfloor n s v\rfloor+q \sqrt{n}\right) P\left(X_{n t}^{m}>\lfloor n t v\rfloor+r \sqrt{n}\right)\right] \\
& +n^{-1 / 2} \sigma_{0}^{2} P\left(X_{n s}^{m}>\lfloor n s v\rfloor+q \sqrt{n}\right) P\left(X_{n t}^{m}>\lfloor n t v\rfloor+r \sqrt{n}\right) .
\end{aligned}
$$

Do the same for the $V$-terms. After some rearranging of the probabilities, we arrive at

$$
\begin{aligned}
& \sum_{|m| \leq a(n) \sqrt{n}} E\left[u(m)^{2}\right] \\
& =n^{-1 / 2} \sum_{1 \leq i, j \leq N} \theta_{i} \theta_{j}\left[\mu _ { 0 } \sum _ { | m | \leq a ( n ) \sqrt { n } } \left\{P\left(X_{n t_{i}}^{m} \leq\left\lfloor n t_{i} v\right\rfloor+r_{i} \sqrt{n}\right)\right.\right. \\
& \times P\left(X_{n t_{j}}^{m}>\left\lfloor n t_{j} v\right\rfloor+r_{j} \sqrt{n}\right) \\
& \left.-P\left(X_{n t_{i}}^{m} \leq\left\lfloor n t_{i} v\right\rfloor+r_{i} \sqrt{n}, X_{n t_{j}}^{m}>\left\lfloor n t_{j} v\right\rfloor+r_{j} \sqrt{n}\right)\right\} \\
& +\sigma_{0}^{2} \sum_{-a(n) \sqrt{n} \leq m \leq 0} P\left(X_{n t_{i}}^{m}>\left\lfloor n t_{i} v\right\rfloor+r_{i} \sqrt{n}\right) P\left(X_{n t_{j}}^{m}>\left\lfloor n t_{j} v\right\rfloor+r_{j} \sqrt{n}\right) \\
& \left.+\sigma_{0}^{2} \sum_{0<m \leq a(n) \sqrt{n}} P\left(X_{n t_{i}}^{m} \leq\left\lfloor n t_{i} v\right\rfloor+r_{i} \sqrt{n}\right) P\left(X_{n t_{j}}^{m} \leq\left\lfloor n t_{j} v\right\rfloor+r_{j} \sqrt{n}\right)\right] .
\end{aligned}
$$

The terms above have been arranged so that the sums match up with the integrals in (2.20)-(2.22). Limit (2.24) now follows because each sum 
converges to the corresponding integral. To illustrate with the last term, the convergence needed is

$$
\begin{gathered}
n_{0<m \leq a(n) \sqrt{n}} \sum_{0 n_{n s}} P\left(X_{n s}^{m} \leq\lfloor n s v\rfloor+q \sqrt{n}\right) P\left(X_{n t}^{m} \leq\lfloor n t v\rfloor+r \sqrt{n}\right) \\
\sum_{0<m \leq a(n) \sqrt{n}} P\left\{\frac{X_{n s}-\lfloor n s v\rfloor}{\sqrt{n}} \leq q-\frac{m}{\sqrt{n}}\right\} \\
\times P\left\{\frac{X_{n t}-\lfloor n t v\rfloor}{\sqrt{n}} \leq r-\frac{m}{\sqrt{n}}\right\} \\
\underset{n \rightarrow \infty}{\longrightarrow} \int_{0}^{\infty} \mathbf{P}\left[B_{\sigma_{1}^{2} s} \leq q-x\right] \mathbf{P}\left[B_{\sigma_{1}^{2} t} \leq r-x\right] d x .
\end{gathered}
$$

This follows from the CLT, a Riemann sum type argument and some estimation. We skip the details. With this we consider Theorem 2.2 proved.

\section{References}

The results for i.i.d. walks appeared, with a slightly different definition of the current process, in [Sep05] and [Kum08]. Earlier related results appeared in [DGL85]. 


\section{Chapter 3}

\section{Independent particles in a random environment}

In this chapter we generalize the results of Chapter 2 to particles in a random environment, with the purpose of seeing how the environment influences the outcome. In a fixed environment, that is, conditional on the environment, the particles evolve independently. But under the joint distribution of the walks and the environment, the particles are no longer independent because their evolution gives information about the environment. The environment is static, which means that it is fixed in time.

\subsection{Model and results}

We formulate the standard one-dimensional nearest-neighbor random walk in random environment (RWRE) model and then put many particles in a fixed environment. We describe the (known) law of large numbers and central limit theorem of the walk itself, and then the (newer) results on current fluctuations for many particles.

The space of environments is $\Omega=[0,1]^{\mathbb{Z}}$. For an environment $\omega=$ $\left\{\omega_{x}\right\}_{x \in \mathbb{Z}} \in \Omega$ let $\left\{X_{.}^{m, i}\right\}_{m, i}$ be a family of Markov chains on $\mathbb{Z}$ with distribution $P_{\omega}$ determined by the following properties:

1. $\left\{X^{m, i}\right\}_{m \in \mathbb{Z}, i \in \mathbb{N}}$ are independent under the measure $P_{\omega}$.

2. $P_{\omega}\left(X_{0}^{m, i}=m\right)=1$, for all $m \in \mathbb{Z}$ and $i \in \mathbb{N}$.

3. Each walk obeys these transition probabilities:

$$
P_{\omega}\left(X_{n+1}^{m, i}=x+1 \mid X_{n}^{m, i}=x\right)=1-P_{\omega}\left(X_{n+1}^{m, i}=x-1 \mid X_{n}^{m, i}=x\right)=\omega_{x} .
$$


A system of random walks in a random environment may then be constructed by first choosing an environment $\omega$ according to a probability distribution $P$ on $\Omega$ and then constructing the system of random walks $\left\{X_{.}^{m, i}\right\}$ as described above. The distribution $P_{\omega}$ of the random walks given the environment $\omega$ is called the quenched distribution. The averaged distribution $\mathbb{P}$ (also called annealed) is obtained by averaging the quenched law over all environments: $\mathbb{P}(\cdot)=\int_{\Omega} P_{\omega}(\cdot) P(d \omega)$. Expectations with respect to the measures $P, P_{\omega}$ and $\mathbb{P}$ are denoted by $E_{P}, E_{\omega}$, and $\mathbb{E}$, respectively, and variances with respect to the measure $P_{\omega}$ will be denoted by $\operatorname{Var}_{\omega}$. We make the following assumptions on the environment.

Assumption 1. The distribution $P$ on environments is i.i.d. and uniformly elliptic. That is, $\left\{\omega_{x}\right\}_{x \in \mathbb{Z}}$ are i.i.d. under the measure $P$, and there exists a $\kappa>0$ such that $P\left(\omega_{x} \in[\kappa, 1-\kappa]\right)=1$. Furthermore, $E_{P}\left(\rho_{0}^{2+\varepsilon_{0}}\right)<1$ for some $\varepsilon_{0}>0$, where $\rho_{x}=\left(1-\omega_{x}\right) / \omega_{x}$.

These assumptions put the RWRE in the regime where it has transience to $+\infty$ with a strictly positive speed and also satisfies a CLT with an environment-dependent centering. We summarize these results here. Define a shift map on environments by $\left(\theta^{x} \omega\right)_{y}=\omega_{x+y}$. Let $T_{1}=\inf \{n \geq 0$ : $\left.X_{n}=1\right\}$ be the first hitting time of site $1 \in \mathbb{Z}$ by a RWRE started at the origin, and define

$$
Z_{n t}(\omega)=\mathrm{v}_{P} \sum_{i=0}^{\left\lfloor n t \mathrm{v}_{P}\right\rfloor-1}\left(E_{\theta^{i} \omega}\left(T_{1}\right)-\mathbb{E} T_{1}\right)
$$

The asymptotic speed $\mathrm{v}_{P}$ is defined in the first statement of the next theorem where we summarize some known basic facts about RWRE.

Theorem 3.1 ([Sol75, Pet08, Zei04]). Under the assumptions made above we have these conclusions.

1. The RWRE satisfies a law of large numbers with positive speed. That is,

$$
\lim _{n \rightarrow \infty} \frac{X_{n}}{n}=\frac{1-E_{P}\left(\rho_{0}\right)}{1+E_{P}\left(\rho_{0}\right)} \equiv \mathrm{v}_{P}>0, \quad \mathbb{P} \text {-a.s. }
$$

2. The RWRE satisfies a quenched functional central limit theorem with an environment-dependent centering. Let

$$
B^{n}(t)=\frac{X_{n t}-n t \mathrm{v}_{P}+Z_{n t}(\omega)}{\sigma_{1} \sqrt{n}}, \quad \text { where } \sigma_{1}^{2}=\mathrm{v}_{P}^{3} E_{P}\left(\operatorname{Var}_{\omega} T_{1}\right)
$$

Then, for $P$-a.e. environment $\omega$, under the quenched measure $P_{\omega}$, $B^{n}(\cdot)$ converges weakly to standard Brownian motion as $n \rightarrow \infty$. 
3. Let

$$
\zeta^{n}(t)=\frac{Z_{n t}(\omega)}{\sigma_{2} \sqrt{n}}, \quad \text { where } \sigma_{2}^{2}=\mathrm{v}_{P}^{2} \operatorname{Var}\left(E_{\omega} T_{1}\right) .
$$

Then, under the measure $P$ on environments, $\zeta^{n}(\cdot)$ converges weakly to standard Brownian motion as $n \rightarrow \infty$.

4. The RWRE satisfies an averaged central limit theorem. Let

$$
\mathbb{B}^{n}(t)=\frac{X_{n t}-n t \mathrm{v}_{P}}{\sigma \sqrt{n}}, \quad \text { where } \sigma^{2}=\sigma_{1}^{2}+\sigma_{2}^{2} .
$$

Then, under the averaged measure $\mathbb{P}, \mathbb{B}^{n}(\cdot)$ converges weakly to standard Brownian motion.

The requirement that $E_{P}\left(\rho_{0}^{2}\right)<1$ cannot be relaxed in order for the CLT to hold [KKS75, PZ09]. Centering by $n t \mathrm{v}_{P}-Z_{n t}(\omega)$ in the quenched CLT is the same as centering by the quenched mean on account of this bound:

$$
\lim _{n \rightarrow \infty} P\left\{\omega: \sup _{k \leq n}\left|E_{\omega}\left(X_{k}\right)-k \mathrm{v}_{P}+Z_{k}(\omega)\right| \geq \varepsilon \sqrt{n}\right\}=0, \quad \forall \varepsilon>0 .
$$

But $Z_{n t}(\omega)$ is more convenient because it is a sum of stationary, ergodic random variables.

These properties of the walk are sufficient for describing the current fluctuations. Assumptions on the initial occupation variables $\eta(0)=\left\{\eta_{x}(0)\right\}$ are similar to those in the previous section. We will allow the distribution of $\eta(0)$ to depend on the environment (in a measurable way), and we assume a certain stationarity.

Assumption 2. Given the environment $\omega$, variables $\left\{\eta_{x}(0)\right\}$ are independent and independent of the random walks. The conditional distribution of $\eta_{x}(0)$ given $\omega$ is denoted by $P_{\omega}\left(\eta_{x}(0)=k\right)$, and these measurable functions of $\omega$ satisfy $P_{\omega}\left(\eta_{x}(0)=k\right)=P_{\theta^{x}}\left(\eta_{0}(0)=k\right)$. Also, for some $\varepsilon_{0}>0$,

$$
E_{P}\left[E_{\omega}\left(\eta_{x}(0)\right)^{2+\varepsilon_{0}}+\operatorname{Var}_{\omega}\left(\eta_{x}(0)\right)^{2+\varepsilon_{0}}\right]<\infty .
$$

Let

$$
\mu_{0}=E_{P}\left[E_{\omega}\left(\eta_{x}(0)\right)\right]=\mathbb{E}\left[\eta_{x}(0)\right] \quad \text { and } \quad \sigma_{0}^{2}=E_{P}\left[\operatorname{Var}_{\omega}\left(\eta_{x}(0)\right)\right] .
$$

The current is defined as before:

$$
\begin{aligned}
Y_{n}(t, r)=\sum_{m \leq 0} \sum_{k=1}^{\eta_{m}(0)} \mathbf{1}\left\{X_{n t}^{m, k}>\left\lfloor n t \mathrm{v}_{P}\right\rfloor+r \sqrt{n}\right\} \\
\quad-\sum_{m>0} \sum_{k=1}^{\eta_{m}(0)} \mathbf{1}\left\{X_{n t}^{m, k} \leq\left\lfloor n t \mathrm{v}_{P}\right\rfloor+r \sqrt{n}\right\} .
\end{aligned}
$$


Now for the results, beginning with the quenched mean of the current. This turns out to essentially follow the correction $Z_{n t}(\omega)$ of the quenched CLT, which is of order $\sqrt{n}$. Assumptions 1 and 2 are in force for all the results that follow.

Theorem 3.2. For any $\varepsilon>0,0<R, T<\infty$,

$$
\lim _{n \rightarrow \infty} P\left\{\omega: \sup _{\substack{t \in[0, T] \\ r \in[-R, R]}}\left|E_{\omega} Y_{n}(t, r)+\mu_{0} r \sqrt{n}+\mu_{0} Z_{n t}(\omega)\right| \geq \varepsilon \sqrt{n}\right\}=0
$$

Consequently the two-parameter process $\left\{n^{-1 / 2} E_{\omega} Y_{n}(t, r): t \in \mathbb{R}_{+}, r \in\right.$ $\mathbb{R}\}$ converges weakly to $\left\{-\mu_{0} r+\mu_{0} \sigma_{2} W(t): t \in \mathbb{R}_{+}, r \in \mathbb{R}\right\}$ where $W(\cdot)$ is a standard Brownian motion.

Next we center the current at its quenched mean by defining

$$
V_{n}(t, r)=Y_{n}(t, r)-E_{\omega}\left[Y_{n}(t, r)\right]
$$

The fluctuations of $V_{n}(t, r)$ are of order $n^{1 / 4}$ and similar to the current fluctuations of classical walks from the previous section. Recall the definitions of $\Gamma_{1}$ and $\Gamma_{2}$ from $(2.10)-(2.11)$ and abbreviate

$$
\Gamma((s, q),(t, r))=\mu_{0} \Gamma_{1}((s, q),(t, r))+\sigma_{0}^{2} \Gamma_{2}((s, q),(t, r)) .
$$

Let $(V, Z)=\left(V(t, r), Z(t): t \in \mathbb{R}_{+}, r \in \mathbb{R}\right)$ be the process whose joint distribution is defined as follows:

(i) Marginally, $Z(\cdot)=\sigma_{2} W(\cdot)$ for a standard Brownian motion $W(\cdot)$.

(ii) Conditionally on the path $Z(\cdot) \in C\left(\mathbb{R}_{+}, \mathbb{R}\right), V$ is the mean zero Gaussian process indexed by $\mathbb{R}_{+} \times \mathbb{R}$ with covariance

$$
\mathbf{E}[V(s, q) V(t, r) \mid Z(\cdot)]=\Gamma((s, q+Z(s)),(t, r+Z(t)))
$$

for $(s, q),(t, r) \in \mathbb{R}_{+} \times \mathbb{R}$. An equivalent way to say this is to first take independent $\left(V^{0}, Z\right)$ with $Z$ as above and $V^{0}=\left\{V^{0}(t, r):(t, r) \in \mathbb{R}_{+} \times \mathbb{R}\right\}$ the mean zero Gaussian process with covariance $\Gamma((s, q),(t, r))$ from $(3.7)$, and then define $V(t, r)=V^{0}(t, r+Z(t))$.

Theorem 3.3. Under the averaged probability $\mathbb{P}$, as $n \rightarrow \infty$, the finitedimensional distributions of the joint process $\left\{\left(n^{-1 / 4} V_{n}(t, r), n^{-1 / 2} Z_{n t}(\omega)\right)\right.$ : $t \in \mathbb{R}_{+}, r \in \mathbb{R}_{\}}$converge to those of the process $(V, Z)$.

Thus up to a random shift of the spatial argument we see the same limit process as for classical walks: the process $\bar{V}(t, r)=V(t, r-Z(t))$ is a mean 
zero Gaussian process with covariance $\mathbf{E}[\bar{V}(s, q) \bar{V}(t, r)]=\Gamma((s, q),(t, r))$ from (3.7).

As for classical walks, let us look at the stationary case. The invariant distribution is now valid under a fixed $\omega$ : the $\left\{\eta_{x}(0)\right\}$ are independent and

$$
\eta_{x}(0) \sim \operatorname{Poisson}\left(\mu_{0} f\left(\theta^{x} \omega\right)\right), \quad \text { where } f(\omega)=\frac{\mathrm{v}_{P}}{\omega_{0}}\left(1+\sum_{i=1}^{\infty} \prod_{j=1}^{i} \rho_{j}\right)
$$

In this case, $E_{\omega} \eta_{0}(0)=\operatorname{Var}_{\omega} \eta_{0}(0)=\mu_{0} f(\omega)$. By Assumption $1 \quad E_{P}\left(\rho_{0}^{2+\varepsilon}\right)$ $<1$ for some $\varepsilon>0$, and from that it can be shown that $E_{P}\left[f(\omega)^{2+\varepsilon}\right]<\infty$. Therefore Assumption 2 holds. One can also check that, as for classical walks in (2.3), in this stationary situation the flux is linear: $H\left(\mu_{0}\right)=\mu_{0} \mathrm{v}_{P}$.

Recall from Corollary 2.1 that for classical random walks the limit process (with fixed space variable $r$ ) in the case $\mu_{0}=\sigma_{0}^{2}$ is fractional Brownian motion $\xi$ with covariance

$$
\mathbf{E}[\xi(s) \xi(t)]=\frac{\mu_{0} \sigma_{1}}{\sqrt{2 \pi}}(\sqrt{s}+\sqrt{t}-\sqrt{|s-t|}) .
$$

For RWRE, $\mu_{0}=\sigma_{0}^{2}$ implies that

$$
\begin{aligned}
& \mathbf{E}[V(s, 0) V(t, 0) \mid Z(\cdot)] \\
& =\mu_{0}\left[\Psi_{\sigma_{1}^{2} s}(-Z(s))+\Psi_{\sigma_{1}^{2} t}(Z(t))-\Psi_{\sigma_{1}^{2}|s-t|}(Z(t)-Z(s))\right] .
\end{aligned}
$$

Since the right hand side of (3.10) is a non-constant random variable, the marginal distribution of $V(t, 0)$ is non-Gaussian. Taking expectations of (3.10) with respect to $Z(\cdot)$ gives that

$$
\mathbf{E}[V(s, 0) V(t, 0)]=\frac{\mu_{0} \sqrt{\sigma_{1}^{2}+\sigma_{2}^{2}}}{\sqrt{2 \pi}}(\sqrt{s}+\sqrt{t}-\sqrt{|s-t|}) .
$$

Thus we get this conclusion: if $\mu_{0}=\sigma_{0}^{2}$ for RWRE then the limit process $V(\cdot, 0)$ has the same covariance as fractional Brownian motion, but it is not a Gaussian process.

As the reader may have surmised, we can remove the random shift $Z$ from the limit process $V$ by introducing the environment-dependent shift in the current process itself. We state this result too. For $(t, r) \in \mathbb{R}_{+} \times \mathbb{R}$ define

$$
\begin{aligned}
Y_{n}^{(q)}(t, r)=\sum_{m>0} \sum_{k=1}^{\eta_{m}(0)} \mathbf{1}\left\{X_{n t}^{m, k} \leq n t \mathrm{v}_{P}-Z_{n t}(\omega)+r \sqrt{n}\right\} \\
-\sum_{m \leq 0} \sum_{k=1}^{\eta_{m}(0)} \mathbf{1}\left\{X_{n t}^{m, k}>n t \mathrm{v}_{P}-Z_{n t}(\omega)+r \sqrt{n}\right\}
\end{aligned}
$$


and its centered version

$$
V_{n}^{(q)}(t, r)=Y_{n}^{(q)}(t, r)-E_{\omega} Y_{n}^{(q)}(t, r) .
$$

The process $V_{n}^{(q)}$ has the same limit as classical random walks. Let $V^{0}=$ $\left\{V^{0}(t, r):(t, r) \in \mathbb{R}_{+} \times \mathbb{R}\right\}$ be the mean zero Gaussian process with covariance $\Gamma((s, q),(t, r))$ from $(3.7)$.

Theorem 3.4. Under the averaged probability $\mathbb{P}$, as $n \rightarrow \infty$, the finitedimensional distributions of the joint process $\left\{\left(n^{-1 / 4} V_{n}^{(q)}(t, r), n^{-1 / 2} Z_{n t}(\omega)\right)\right.$ : $\left.t \in \mathbb{R}_{+}, r \in \mathbb{R}\right\}$ converge to those of the process $\left(V^{0}, Z\right)$ where $V^{0}$ and $Z$ are independent.

\subsection{Sketch of the proof for the quenched mean of the current}

The basic thrust of the proof of Theorem 3.3 for the limit of the centered current is similar to the one outlined in Section 2.2 for classical random walks. The differences lie in the technical details needed to handle the random environment. So we omit further discussion of that theorem and of the related Theorem 3.4. In this section we explain the main ideas behind the proof of Theorem 3.2 for the quenched mean of the current. To put aside inessential detail we drop the uniformity, fix $(t, r)$, and sketch informally the argument for the following simplified statement:

Proposition 3.1. For any $\varepsilon>0$,

$$
\lim _{n \rightarrow \infty} P\left\{\omega:\left|E_{\omega} Y_{n}(t, r)+\mu_{0} r \sqrt{n}+\mu_{0} Z_{n t}(\omega)\right| \geq \varepsilon \sqrt{n}\right\}=0 .
$$

This proceeds via a sequence of estimations. Abbreviate the centered quenched mean by

$$
\begin{aligned}
W_{n}= & E_{\omega} Y_{n}(t, r)+\mu_{0} r \sqrt{n} \\
= & \sum_{m \leq 0} E_{\omega}\left[\eta_{0}(m)\right] P_{\omega}\left(X_{n t}^{m}>n t \mathrm{v}_{P}+r \sqrt{n}\right) \\
& \quad-\sum_{m>0} E_{\omega}\left[\eta_{0}(m)\right] P_{\omega}\left(X_{n t}^{m} \leq n t \mathrm{v}_{P}+r \sqrt{n}\right)+\mu_{0} r \sqrt{n} .
\end{aligned}
$$

For a suitable sequence $a(n) \nearrow \infty$ define

$$
\begin{aligned}
\widetilde{W}_{n}= & \sum_{m=-\lfloor a(n) \sqrt{n}\rfloor+1}^{0} E_{\omega}\left(\eta_{0}(m)\right) \Phi_{\sigma_{1}^{2} t}\left(-\frac{Z_{n t}\left(\theta^{m} \omega\right)-m}{\sqrt{n}}-r\right) \\
& -\sum_{m=1}^{\lfloor a(n) \sqrt{n}\rfloor} E_{\omega}\left(\eta_{0}(m)\right) \Phi_{\sigma_{1}^{2} t}\left(\frac{Z_{n t}\left(\theta^{m} \omega\right)-m}{\sqrt{n}}+r\right)+\mu_{0} r \sqrt{n} .
\end{aligned}
$$


The quenched CLT (part 2 of Theorem 3.1) implies that the difference

$$
\sup _{x \in \mathbb{R}}\left|P_{\omega}\left(\frac{X_{n}-n \mathrm{v}_{P}+Z_{n}(\omega)}{\sqrt{n}} \leq x\right)-\Phi_{\sigma_{1}^{2}}(x)\right|
$$

vanishes $P$-a.s. as $n \rightarrow \infty$. Thus it is possible to choose $a(n) \nearrow \infty$ slowly enough so that

$$
\lim _{n \rightarrow \infty} P\left(\frac{1}{\sqrt{n}}\left|W_{n}-\widetilde{W}_{n}\right| \geq \varepsilon\right)=0 .
$$

The next step is to remove the shifts from $Z_{n t}$ by defining

$$
\begin{aligned}
\widehat{W}_{n}= & \sum_{m=-\lfloor a(n) \sqrt{n}\rfloor+1}^{0} E_{\omega}\left(\eta_{0}(m)\right) \Phi_{\sigma_{1}^{2} t}\left(-\frac{Z_{n t}(\omega)-m}{\sqrt{n}}-r\right) \\
& -\sum_{m=1}^{\lfloor a(n) \sqrt{n}\rfloor} E_{\omega}\left(\eta_{0}(m)\right) \Phi_{\sigma_{1}^{2} t}\left(\frac{Z_{n t}(\omega)-m}{\sqrt{n}}+r\right)+\mu_{0} r \sqrt{n} .
\end{aligned}
$$

The estimation

$$
\lim _{n \rightarrow \infty} P\left(\frac{1}{\sqrt{n}}\left|\widetilde{W}_{n}-\widehat{W}_{n}\right| \geq \varepsilon\right)=0
$$

follows from representation (3.1) of $Z_{n t}$ as a sum of ergodic terms whose behavior is well understood.

Subsequently the ergodicity of the environment allows us to average the quenched means $E_{\omega}\left(\eta_{0}(m)\right)$ and replace $\widehat{W}_{n}$ with

$$
\begin{aligned}
\bar{W}_{n}= & \sum_{m=-\lfloor a(n) \sqrt{n}\rfloor+1}^{0} \mu_{0} \Phi_{\sigma_{1}^{2} t}\left(-\frac{Z_{n t}(\omega)-m}{\sqrt{n}}-r\right) \\
& -\sum_{m=1}^{\lfloor a(n) \sqrt{n}\rfloor} \mu_{0} \Phi_{\sigma_{1}^{2} t}\left(\frac{Z_{n t}(\omega)-m}{\sqrt{n}}+r\right)+\mu_{0} r \sqrt{n} .
\end{aligned}
$$

After this, replace the sums with integrals to approximate $n^{-1 / 2} \bar{W}_{n}$ with

$$
\mu_{0} r-\mu_{0} \int_{0}^{a(n)} \Phi_{\sigma_{1}^{2} t}\left(\frac{Z_{n t}(\omega)}{\sqrt{n}}+r-x\right)-\Phi_{\sigma_{1}^{2} t}\left(-\frac{Z_{n t}(\omega)}{\sqrt{n}}-r-x\right) d x .
$$

A calculus exercise shows that

$$
\int_{0}^{A} \Phi_{\alpha^{2}}(z-x)-\Phi_{\alpha^{2}}(-z-x) d x=z+\Psi_{\alpha^{2}}(A+z)-\Psi_{\alpha^{2}}(A-z),
$$


where $\Psi_{\alpha^{2}}(x)$ is again the function defined in (2.9). Therefore,

$$
\begin{gathered}
\int_{0}^{a(n)} \Phi_{\sigma_{1}^{2} t}\left(\frac{Z_{n t}(\omega)}{\sqrt{n}}+r-x\right)-\Phi_{\sigma_{1}^{2} t}\left(-\frac{Z_{n t}(\omega)}{\sqrt{n}}-r-x\right) d x \\
=\frac{Z_{n t}(\omega)}{\sqrt{n}}+r+\Psi_{\sigma_{1}^{2} t}\left(a(n)+\frac{Z_{n t}(\omega)}{\sqrt{n}}+r\right) \\
-\Psi_{\sigma_{1}^{2} t}\left(a(n)-\frac{Z_{n t}(\omega)}{\sqrt{n}}-r\right) .
\end{gathered}
$$

The last two terms vanish as $n \rightarrow \infty$ because $\Psi_{\sigma_{1}^{2} t}(\infty)=0$ and $n^{-1 / 2} Z_{n t}$ is tight by part 3 of Theorem 3.1 .

Collecting the steps (and taking the omitted details on faith) leads to the estimate

$$
W_{n}=-\mu_{0} Z_{n t}+o(\sqrt{n}) \quad \text { in probability }
$$

as was claimed in (3.13).

\section{References}

The results for the current of RWRE's are from [PS]. Zeitouni's lecture notes [Zei04] are a standard reference for background on RWRE. 


\section{Chapter 4}

\section{Random average process}

\subsection{Model and results}

The state of the random average process (RAP) is a height function $\sigma$ : $\mathbb{Z} \rightarrow \mathbb{R}$ where the value $\sigma(i)$ can be thought of as the height of an interface above site $i$. The state evolves in discrete time according to the following rule. At each time point $s=1,2,3, \ldots$ and at each site $k \in \mathbb{Z}$, a random probability vector $\omega_{k, s}=\left(\omega_{k, s}(j):-R \leq j \leq R\right)$ of length $2 R+1$ is drawn. Given the state $\sigma_{s-1}=\left(\sigma_{s-1}(i): i \in \mathbb{Z}\right)$ at time $s-1$, at time $s$ the height value at site $k$ is updated to

$$
\sigma_{s}(k)=\sum_{j:|j| \leq R} \omega_{k, s}(j) \sigma_{s-1}(k+j) .
$$

This update is performed independently at each site $k$ to form the state $\sigma_{s}=\left(\sigma_{s}(k): k \in \mathbb{Z}\right)$ at time $s$. The weight vectors $\left\{\omega_{k, s}\right\}_{k \in \mathbb{Z}, s \in \mathbb{N}}$ are i.i.d. across space-time points $(k, s)$. This system was originally studied by Ferrari and Fontes [FF98].

Let

$$
p(0, j)=\mathbb{E}\left[\omega_{0,0}(j)\right]
$$

denote the averaged weights with mean and variance

$$
V=\sum_{x} x p(x) \quad \text { and } \quad \sigma_{1}^{2}=\sum_{x \in \mathbb{Z}}(x-V)^{2} p(0, x) .
$$

Let $b=-V$.

Make two nondegeneracy assumptions on the distribution of the weight vectors.

(i) There is no integer $h>1$ such that, for some $x \in \mathbb{Z}$,

$$
\sum_{k \in \mathbb{Z}} p(0, x+k h)=1 \text {. }
$$


This is also expressed by saying that the span of the random walk with jump probabilities $p(0, j)$ is 1 [Dur04, page 129]. It follows that the additive group generated by $\{x \in \mathbb{Z}: p(0, x)>0\}$ is all of $\mathbb{Z}$, in other words this walk is aperiodic in Spitzer's terminology [Spi76].

(ii) Second, we assume that

$$
\mathbb{P}\left\{\max _{j} \omega_{0,0}(j)<1\right\}>0
$$

Let $\sigma_{s}$ be a random average process normalized by $\sigma_{0}(0)=0$ and whose initial increments $\left\{\eta_{i}(0)=\sigma_{0}(i)-\sigma_{0}(i-1): i \in \mathbb{Z}\right\}$ are i.i.d. such that

$$
\text { there exists } \alpha>0 \text { such that } E\left[\left|\eta_{i}(0)\right|^{2+\alpha}\right]<\infty \text {. }
$$

As before, the mean and variance of initial increments are

$$
\mu_{0}=E\left(\eta_{i}(0)\right) \quad \text { and } \quad \sigma_{0}^{2}=\operatorname{Var}\left(\eta_{i}(0)\right) .
$$

The initial increments $\eta_{0}$ are independent of the weight vectors $\left\{\omega_{k, s}\right\}$.

Again we study a suitably scaled process of fluctuations in the characteristic direction: for $(t, r) \in \mathbb{R}_{+} \times \mathbb{R}$, let

$$
\bar{Y}_{n}(t, r)=n^{-1 / 4}\left\{\sigma_{\lfloor n t\rfloor}^{n}(\lfloor r \sqrt{n}\rfloor+\lfloor n t b\rfloor)-\mu_{0} r \sqrt{n}\right\} .
$$

In terms of the increment process

$$
\eta_{i}(s)=\sigma_{s}(i)-\sigma_{s}(i-1),
$$

$\bar{Y}_{n}(t, r)$ is the centered and scaled net flow from right to left across the path $s \mapsto\lfloor r \sqrt{n}\rfloor+\lfloor n s b\rfloor$, during the time interval $0 \leq s \leq t$, exactly as for independent particles.

Recall the definitions (2.10) and (2.11) of the functions $\Gamma_{1}$ and $\Gamma_{2}$.

Theorem 4.1. Under the above assumptions the finite-dimensional distributions of the process $\left\{\bar{Y}_{n}(t, r):(t, r) \in \mathbb{R}_{+} \times \mathbb{R}\right\}$ converge weakly as $n \rightarrow \infty$ to the finite-dimensional distributions of the mean zero Gaussian process $\left\{Z(t, r):(t, r) \in \mathbb{R}_{+} \times \mathbb{R}\right\}$ specified by the covariance

$$
\mathbf{E} Z(s, q) Z(t, r)=\mu_{0}^{2} \kappa \Gamma_{1}((s, q),(t, r))+\sigma_{0}^{2} \Gamma_{2}((s, q),(t, r)) .
$$

The constant $\kappa$ is determined by the distribution of the random weights and will be described precisely later in equation (4.29).

Invariant distributions for the general RAP are not known. The next example may be the only one where explicit invariant distributions are available. 
Example 4.2. Fix positive real parameters $\theta>\alpha>0$. Let $\left\{\omega_{k, s}(-1)\right.$ : $s \in \mathbb{N}, k \in \mathbb{Z}\}$ be i.i.d. $\operatorname{Beta}(\alpha, \theta-\alpha)$ random variables with density

$$
h(u)=\frac{\Gamma(\theta)}{\Gamma(\alpha) \Gamma(\theta-\alpha)} u^{\alpha-1}(1-u)^{\theta-\alpha-1}
$$

on $(0,1)$. Set $\omega_{k, s}(0)=1-\omega_{k, s}(-1)$. Thus the weights are supported on $\{-1,0\}$. A family of invariant distributions for the increment process $\eta(s)=\left(\eta_{k}(s): k \in \mathbb{Z}\right)$ is obtained by letting the variables $\left\{\eta_{k}: k \in \mathbb{Z}\right\}$ be i.i.d. $\operatorname{Gamma}(\theta, \lambda)$ distributed with common density

$$
f(x)=\frac{1}{\Gamma(\theta)} \lambda e^{-\lambda x}(\lambda x)^{\theta-1}
$$

on $\mathbb{R}_{+}$. This family of invariant distributions is parametrized by $0<\lambda<$ $\infty$. Under this distribution $E^{\lambda}\left[\eta_{k}\right]=\theta / \lambda$ and $\operatorname{Var}^{\lambda}\left[\eta_{k}\right]=\theta / \lambda^{2}$. In this situation we find again the fractional Brownian motion limit:

$$
\mathbf{E} Z(s, 0) Z(t, 0)=c_{1}(\sqrt{s}+\sqrt{t}-\sqrt{|t-s|}) .
$$

for a certain constant $c_{1}$.

\subsection{Steps of the proof}

\section{Representation in terms of space-time RWRE}

Let $\omega=\left(\omega_{k, s}: s \in \mathbb{N}, k \in \mathbb{Z}\right)$ represent the i.i.d. random weight vectors that determine the dynamics, coming from a probability space $(\Omega, \mathfrak{S}, \mathbb{P})$. Given $\omega$ and a space-time point $(i, \tau)$, let $\left\{X_{s}^{i, \tau}: s \in \mathbb{Z}_{+}\right\}$denote a random walk on $\mathbb{Z}$ that starts at $X_{0}^{i, \tau}=i$ and whose transition probabilities are given by

$$
P^{\omega}\left(X_{s+1}^{i, \tau}=y \mid X_{s}^{i, \tau}=x\right)=\omega_{x, \tau-s}(y-x) .
$$

$P^{\omega}$ is the path measure of the walk $X_{s}^{i, \tau}$, with expectation denoted by $E^{\omega}$. Comparison of (4.1) and (4.8) gives

$$
\sigma_{s}(i)=\sum_{j} P^{\omega}\left(X_{1}^{i, s}=j \mid X_{0}^{i, s}=i\right) \sigma_{s-1}(j)=E^{\omega}\left[\sigma_{s-1}\left(X_{1}^{i, s}\right)\right] .
$$

Iteration and the Markov property of the walks $X_{s}^{i, s}$ then lead to

$$
\sigma_{s}(i)=E^{\omega}\left[\sigma_{0}\left(X_{s}^{i, s}\right)\right] .
$$

Note that the initial height function $\sigma_{0}$ is a constant under the expectation $E^{\omega}$.

Let us add another coordinate to keep track of time and write $\bar{X}_{s}^{i, \tau}=$ $\left(X_{s}^{i, \tau}, \tau-s\right)$ for $s \geq 0$. Then $\bar{X}_{s}^{i, \tau}$ is a random walk on the planar lattice 
$\mathbb{Z}^{2}$ that always moves down one step in the $e_{2}$-direction, and if its current position is $(x, n)$, then its next position is $(x+y, n-1)$ with probability $\omega_{x, n}(y-x)$. We could call this a backward random walk in a (space-time, or dynamical) random environment.

The opening step of the proof is to use the random walk representation to rewrite the random variable $\bar{Y}_{n}(t, r)$ in a manner that allows us to separate the effects of the random initial conditions from the effects of the random weights. Abbreviate

$$
y(n)=\lfloor n t b\rfloor+\lfloor r \sqrt{n}\rfloor .
$$

and recall $\mu_{0}=E \eta_{i}(0)$ and $\sigma_{0}(0)=0$.

$$
\begin{aligned}
& \sigma_{\lfloor n t\rfloor}(y(n))=E^{\omega}\left[\sigma_{0}\left(X_{\lfloor n t\rfloor}^{y(n),\lfloor n t\rfloor}\right)\right] \\
& =E^{\omega}\left[\mathbf{1}_{\left\{X_{\lfloor n t\rfloor}^{y(n),\lfloor n t\rfloor}>0\right\}} \sum_{i=1}^{X_{\lfloor n t\rfloor}^{y(n)\lfloor n t\rfloor}} \eta_{i}(0)\right. \\
& \left.\quad-\mathbf{1}_{\left\{X_{\lfloor n t\rfloor}^{y(n),\lfloor n t\rfloor}<0\right\}} \sum_{i=X_{\lfloor n t\rfloor}^{y(n)\lfloor n t\rfloor}+1}^{0} \eta_{i}(0)\right] \\
& =\sum_{i>0} \eta_{i}(0) P^{\omega}\left\{i \leq X_{\lfloor n t\rfloor}^{y(n),\lfloor n t\rfloor}\right\}-\sum_{i \leq 0} \eta_{i}(0) P^{\omega}\left\{i>X_{\lfloor n t\rfloor}^{y(n),\lfloor n t\rfloor}\right\} \\
& =\mu_{0} H_{n}(t, r)+S_{n}(t, r)
\end{aligned}
$$

where

$$
\begin{aligned}
H_{n}(t, r)= & \sum_{i \in \mathbb{Z}}\left(\mathbf{1}\{i>0\} P^{\omega}\left\{i \leq X_{\lfloor n t\rfloor}^{y(n),\lfloor n t\rfloor}\right\}\right. \\
& \left.-\mathbf{1}\{i \leq 0\} P^{\omega}\left\{i>X_{\lfloor n t\rfloor}^{y(n),\lfloor n t\rfloor}\right\}\right) \\
= & E^{\omega}\left(X_{\lfloor n t\rfloor}^{y(n),\lfloor n t\rfloor}\right)
\end{aligned}
$$

and

$$
\begin{gathered}
S_{n}(t, r)=\sum_{i \in \mathbb{Z}}\left(\eta_{i}(0)-\mu_{0}\right)\left(1\{i>0\} P^{\omega}\left\{i \leq X_{\lfloor n t\rfloor}^{y(n),\lfloor n t\rfloor}\right\}\right. \\
\left.-\mathbf{1}\{i \leq 0\} P^{\omega}\left\{i>X_{\lfloor n t\rfloor}^{y(n),\lfloor n t\rfloor}\right\}\right) .
\end{gathered}
$$

At this point the terms $H_{n}$ and $S_{n}$ are dependent, but in the course of the scaling limit they become independent and furnish the two independent pieces that make up the limiting process $Z$. The limits $n^{-1 / 4}\left(H_{n}(t, r)-\right.$ 
$r \sqrt{n}) \stackrel{\mathcal{D}}{\longrightarrow} H(t, r)$ and $n^{-1 / 4} S_{n}(t, r) \stackrel{\mathcal{D}}{\longrightarrow} S(t, r)$ are treated separately, and then together

$$
\bar{Y}_{n}=n^{-1 / 4}\left(H_{n}-r \sqrt{n}+S_{n}\right) \stackrel{\mathcal{D}}{\longrightarrow} H+S \equiv Z
$$

with independent terms $H$ and $S$. This independence comes from the independence of the initial height function $\sigma_{0}$ and the random environment $\omega$ that drives the dynamics. The idea is represented in the next lemma.

Lemma 4.1. Let $\eta$ and $\omega$ be independent random variables with values in some abstract measurable spaces. Let $h_{n}(\omega)$ and $s_{n}(\omega, \eta)$ be measurable functions of $(\omega, \eta)$. Let $E^{\omega}(\cdot)$ denote conditional expectation, given $\omega$. Assume the existence of random variables $h$ and $s$ such that

(i) $h_{n}(\omega) \stackrel{\mathcal{D}}{\longrightarrow} h$;

(ii) for all $\theta \in \mathbb{R}, E^{\omega}\left[e^{i \theta s_{n}}\right] \rightarrow E\left(e^{i \theta s}\right)$ in probability as $n \rightarrow \infty$.

Then $h_{n}+s_{n} \stackrel{\mathcal{D}}{\longrightarrow} h+s$, where $h$ and $s$ are independent.

Proof. Let $\theta, \lambda \in \mathbb{R}$. Then

$$
\begin{aligned}
& \left|E\left(E^{\omega}\left[e^{i \lambda h_{n}+i \theta s_{n}}\right]\right)-E\left[e^{i \lambda h}\right] E\left[e^{i \theta s}\right]\right| \\
& \quad \leq\left|E\left[e^{i \lambda h_{n}}\left(E^{\omega} e^{i \theta s_{n}}-E e^{i \theta s}\right)\right]\right|+\left|\left(E e^{i \lambda h_{n}}-E e^{i \lambda h}\right) E e^{i \theta s}\right| \\
& \quad \leq\left|E\left[e^{i \lambda h_{n}}\left(E^{\omega} e^{i \theta s_{n}}-E e^{i \theta s}\right)\right]\right|+\left|E e^{i \lambda h_{n}}-E e^{i \lambda h}\right| .
\end{aligned}
$$

By assumption (i), the second term above goes to 0. By assumption (ii), the integrand in the first term goes to 0 in probability. Therefore by bounded convergence the first term goes to 0 as $n \rightarrow \infty$.

We discuss the term $S_{n}$ briefly and reserve most of our attention to $H_{n}$. Two limits combine to give the result. The idea is to apply the LindebergFeller theorem to $S_{n}(t, r)$ under a fixed $\omega$. Then the $\omega$-dependent coefficients provide no fluctuations but instead converge to Brownian probabilities due to a quenched central limit theorem for the space-time RWRE. Here is an informal presentation where we first imagine that the coefficients 
can be replaced by deterministic quantities:

$$
\begin{aligned}
& S_{n}(t, r)=\sum_{x \in \mathbb{Z}}\left(\eta_{x}(0)-\mu_{0}\right) \\
& \times\left(\mathbf{1}\{x>0\} P^{\omega}\left\{\frac{X_{\lfloor n t\rfloor}^{\lfloor n t b\rfloor+\lfloor r \sqrt{n}\rfloor,\lfloor n t\rfloor}-r \sqrt{n}}{\sqrt{n}} \geq \frac{x}{\sqrt{n}}-r\right\}\right. \\
& \left.\quad-\mathbf{1}\{x \leq 0\} P^{\omega}\left\{\frac{X_{\lfloor n t\rfloor}^{\lfloor n t b\rfloor+\lfloor r \sqrt{n}\rfloor,\lfloor n t\rfloor}-r \sqrt{n}}{\sqrt{n}}<\frac{x}{\sqrt{n}}-r\right\}\right) \\
& \approx \sum_{x \in \mathbb{Z}}\left(\eta_{x}(0)-\mu_{0}\right)\left(\mathbf{1}\{x>0\} \mathbf{P}\left\{B_{\sigma_{1}^{2} t}>\frac{x}{\sqrt{n}}-r\right\}\right. \\
& \left.-\mathbf{1}\{x \leq 0\} \mathbf{P}\left\{B_{\sigma_{1}^{2} t} \leq \frac{x}{\sqrt{n}}-r\right\}\right) .
\end{aligned}
$$

Now apply the Lindeberg-Feller theorem to the remaining sum of independent initial occupation variables, and the limiting covariance comes as:

$$
\begin{aligned}
& n^{-1 / 2} \sum_{i, j} \theta_{i} \theta_{j} E\left[S_{n}\left(t_{i}, r_{i}\right) S_{n}\left(t_{j}, r_{j}\right)\right] \\
& \approx \sigma_{0}^{2} \sum_{i, j} \theta_{i} \theta_{j} n^{-1 / 2}\left[\sum_{x>0} \mathbf{P}\left\{B_{\sigma_{1}^{2} t_{i}}>\frac{x}{\sqrt{n}}-r_{i}\right\} \mathbf{P}\left\{B_{\sigma_{1}^{2} t_{j}}>\frac{x}{\sqrt{n}}-r_{j}\right\}\right. \\
& \left.+\sum_{x \leq 0} \mathbf{P}\left\{B_{\sigma_{1}^{2} t_{i}} \leq \frac{x}{\sqrt{n}}-r_{i}\right\} \mathbf{P}\left\{B_{\sigma_{1}^{2} t_{j}} \leq \frac{x}{\sqrt{n}}-r_{j}\right\}\right] \\
& \approx \sigma_{0}^{2} \sum_{i, j} \theta_{i} \theta_{j}\left[\int_{0}^{\infty} \mathbf{P}\left\{B_{\sigma_{1}^{2} t_{i}}>x-r_{i}\right\} \mathbf{P}\left\{B_{\sigma_{1}^{2} t_{j}}>x-r_{j}\right\} d x\right. \\
& \left.\quad+\int_{-\infty}^{0} \mathbf{P}\left\{B_{\sigma_{1}^{2} t_{i}} \leq x-r_{i}\right\} \mathbf{P}\left\{B_{\sigma_{1}^{2} t_{j}} \leq x-r_{j}\right\} d x\right] \\
& =\sigma_{0}^{2} \sum_{i, j} \theta_{i} \theta_{j} \Gamma_{2}\left(\left(t_{i}, r_{i}\right),\left(t_{j}, r_{j}\right)\right) .
\end{aligned}
$$

Turning this argument rigorous gives the limit in $\mathbb{P}$-probability:

$$
\begin{aligned}
\lim _{n \rightarrow \infty} & E^{\omega}\left[e^{i \sum_{k} \theta_{k} S_{n}\left(t_{k}, r_{k}\right)}\right]=E\left[e^{i \sum_{k} \theta_{k} S\left(t_{k}, r_{k}\right)}\right] \\
& =\exp \left\{-\frac{1}{2} \sigma_{0}^{2} \sum_{i, j} \theta_{i} \theta_{j} \Gamma_{2}\left(\left(t_{i}, r_{i}\right),\left(t_{j}, r_{j}\right)\right)\right\} .
\end{aligned}
$$

In particular, in the limit the fluctuations of $S$ come from the initial occupation variables $\eta_{i}(0)$ and hence are independent of the weights $\omega$ that determine $H_{n}$. 


\section{Quenched mean of the backward space-time RWRE}

The remaining piece of the fluctuations comes from

$$
\bar{H}_{n}(t, r)=n^{-1 / 4} E^{\omega}\left(X_{\lfloor n t\rfloor}^{\lfloor n t b\rfloor+\lfloor r \sqrt{n}\rfloor,\lfloor n t\rfloor}-\lfloor r \sqrt{n}\rfloor\right) .
$$

Theorem 4.3. In the sense of convergence of finite-dimensional distributions, $\bar{H}_{n} \stackrel{\mathcal{D}}{\longrightarrow} H$ where $H(t, r)$ is the mean zero Gaussian process with covariance

$$
\begin{aligned}
\mathbf{E} H(s, q) H(t, r) & =\kappa \Gamma_{1}((s, q),(t, r)) \\
& =\frac{\kappa}{2} \int_{\sigma_{1}^{2}|t-s|}^{\sigma_{1}^{2}(t+s)} \frac{1}{\sqrt{2 \pi v}} \exp \left\{-\frac{1}{2 v}(q-r)^{2}\right\} d v .
\end{aligned}
$$

The constant $\kappa$ is defined below in (4.29).

By comparing covariances (Remark 2.1) one checks that $H$ can also be defined by

$$
H(t, r)=\sqrt{\kappa} \iint_{[0, t] \times \mathbb{R}} \varphi_{\sigma_{1}^{2}(t-s)}(r-z) d W(s, z) .
$$

Formula (4.14) implies that process $\{H(t, r)\}$ is a weak solution for this initial value problem of the stochastic heat equation:

$$
H_{t}=\frac{\sigma_{1}^{2}}{2} H_{r r}+\sqrt{\kappa} \dot{W}, \quad H(0, r) \equiv 0 .
$$

Proof of Theorem 4.3 happens in two steps: first for multiple space points at a fixed time, and then across time points.

\section{Step 1. Martingale increments for fixed time.}

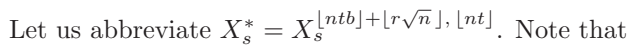

$$
E\left(X_{\lfloor n t\rfloor}^{*}\right)=\lfloor n t b\rfloor+\lfloor r \sqrt{n}\rfloor+\lfloor n t\rfloor V=r \sqrt{n}+O(1)
$$

so $\bar{H}(t, r)$ in (4.12) is essentially centered and we can pretend that it is exactly centered. Let

$$
g(\omega)=E^{\omega}\left(X_{1}^{0,0}\right)-V
$$

be the centered local drift. Recall the space-time walk $\bar{X}_{m}^{x, s}=\left(X_{m}^{x, s}\right.$, $s-m)$. By the Markov property of the walk

$$
\begin{aligned}
& E^{\omega}\left(X_{n}^{x, s}\right)-x-n V=\sum_{k=0}^{n-1} E^{\omega}\left[X_{k+1}^{x, s}-X_{k}^{x, s}-V\right] \\
& =\sum_{k=0}^{n-1} E^{\omega}\left[E^{T_{\left\{X_{k}^{x, s}\right\}} \omega}\left(X_{1}^{0,0}-V\right)\right]=\sum_{k=0}^{n-1} E^{\omega} g\left(T_{\bar{X}_{k}^{x, s}} \omega\right) .
\end{aligned}
$$


$\left(T_{x, m} \omega\right)_{y, s}=\omega_{x+y, m+s}$ is the space-time shift of environments. The $g$ terms above are martingale increments under the distribution $\mathbb{P}$ of the environments, relative to the filtration defined by levels of environments: writing $\bar{\omega}_{m, n}=\left\{\omega_{x, s}: x \in \mathbb{Z}, m \leq s \leq n\right\}$, and with fixed $(x, m)$ and time $n=0,1,2 \ldots$,

$$
\begin{aligned}
\mathbb{E} & {\left[E^{\omega} g\left(T_{\bar{X}_{n}^{x, m}} \omega\right) \mid \bar{\omega}_{m-n+1, m}\right] } \\
& =\sum_{y \in \mathbb{Z}} P^{\omega}\left\{\bar{X}_{n}^{x, m}=(y, m-n)\right\} \int g\left(T_{y, m-n} \omega\right) \mathbb{P}\left(d \bar{\omega}_{m-n}\right)=0 .
\end{aligned}
$$

The point above is that the probability $P^{\omega}\left\{\bar{X}_{n}^{x, m}=(y, m-n)\right\}$ is determined by $\bar{\omega}_{m-n+1, m}$.

It turns out that we can apply a martingale central limit theorem to conclude that, for a fixed $t$, a vector

$$
\left(\bar{H}_{n}\left(t, r_{1}\right), \bar{H}_{n}\left(t, r_{2}\right), \ldots, \bar{H}_{n}\left(t, r_{N}\right)\right)
$$

becomes a Gaussian vector in the $n \rightarrow \infty$ limit. Let us take this for granted, and compute the covariance of the limit. This leads us to study an auxiliary Markov chain which has been useful for space-time (and more general ballistic) RWRE.

Given points $(t, q)$ and $(t, r)$, abbreviate $X_{s}^{(1)}=X_{s}^{\lfloor n t b\rfloor+\lfloor q \sqrt{n}\rfloor,\lfloor n t\rfloor}$ and $X_{s}^{(2)}=X_{s}^{\lfloor n t b\rfloor+\lfloor r \sqrt{n}\rfloor,\lfloor n t\rfloor}$.

$$
\begin{aligned}
& \mathbb{E}\left[\bar{H}_{n}(t, q) \bar{H}_{n}(t, r)\right] \\
& =n^{-1 / 2} \mathbb{E}\left[E^{\omega}\left(X_{\lfloor n t\rfloor}^{(1)}-\lfloor q \sqrt{n}\rfloor\right) E^{\omega}\left(X_{\lfloor n t\rfloor}^{(2)}-\lfloor r \sqrt{n}\rfloor\right)\right] \\
& =n^{-1 / 2} \mathbb{E}\left[\left(\sum_{j=0}^{\lfloor n t\rfloor-1} E^{\omega} g\left(T_{\bar{X}_{j}^{(1)}} \omega\right)\right)\left(\sum_{k=0}^{\lfloor n t\rfloor-1} E^{\omega} g\left(T_{\bar{X}_{k}^{(2)}} \omega\right)\right)\right] \\
& =n^{-1 / 2} \sum_{j, k} \sum_{x, y} \mathbb{E}\left[P^{\omega}\left(X_{j}^{(1)}=x\right) P^{\omega}\left(X_{k}^{(2)}=y\right) g\left(T_{x,\lfloor n t\rfloor-j} \omega\right) g\left(T_{y,\lfloor n t\rfloor-k} \omega\right)\right] \\
& =\sigma_{D}^{2} n^{-1 / 2} \sum_{k=0}^{\lfloor n t\rfloor-1} \mathbb{E} P^{\omega}\left(X_{k}^{(1)}=X_{k}^{(2)}\right) .
\end{aligned}
$$

The last step uses the independence of the environment. We denote the variance of the drift by $\sigma_{D}^{2}=\mathbb{E}\left(g^{2}\right)$. Let now $Y_{k}=X_{k}^{(2)}-X_{k}^{(1)}$ be the difference of two independent walks in a common environment. $Y_{k}$ is a Markov chain on $\mathbb{Z}$ with transition probability

$$
q(x, y)= \begin{cases}\sum_{z \in \mathbb{Z}} \mathbb{E}\left[\omega_{0,0}(z) \omega_{0,0}(0, z+y)\right] & x=0 \\ \sum_{z \in \mathbb{Z}} p(0, z) p(0, z+y-x) & x \neq 0 .\end{cases}
$$


$Y_{n}$ can be thought of as a symmetric random walk on $\mathbb{Z}$ whose transition has been perturbed at the origin. The corresponding homogeneous, unperturbed transition probabilities are

$$
\bar{q}(x, y)=\bar{q}(0, y-x)=\sum_{z \in \mathbb{Z}} p(0, z) p(0, z+y-x) \quad(x, y \in \mathbb{Z}) .
$$

Continuing from above, with $x_{n}=\lfloor r \sqrt{n}\rfloor-\lfloor q \sqrt{n}\rfloor$,

$$
\mathbb{E}\left[\bar{H}_{n}(t, q) \bar{H}_{n}(t, r)\right]=\frac{\sigma_{D}^{2}}{\sqrt{n}} \sum_{k=0}^{\lfloor n t\rfloor-1} q^{k}\left(x_{n}, 0\right)=\frac{\sigma_{D}^{2}}{\sqrt{n}} G_{\lfloor n t\rfloor-1}\left(x_{n}, 0\right) .
$$

If $Y_{k}$ were a symmetric random walk, we would know this limit exactly from the local central limit theorem:

Lemma 4.2. For a mean 0 , span 1 random walk $S_{n}$ on $\mathbb{Z}$ with finite variance $\sigma^{2}, a \in \mathbb{R}$ and points $a_{n} \in \mathbb{Z}$ such that $\left|a_{n}-a \sqrt{n}\right|=O(1)$,

$$
\lim _{n \rightarrow \infty} \frac{1}{\sqrt{n}} \sum_{k=0}^{\lfloor n t\rfloor-1} P\left(S_{k}=a_{n}\right)=\frac{1}{\sigma^{2}} \int_{0}^{\sigma^{2} t} \frac{1}{\sqrt{2 \pi v}} \exp \left\{-\frac{a^{2}}{2 v}\right\} d v .
$$

Proof. By the local CLT [Dur04, Section 2.5]

$$
\lim _{m \rightarrow \infty} \sup _{x \in \mathbb{Z}} \sqrt{m}\left|P\left(S_{m}=x\right)-\frac{1}{\sqrt{2 \pi m \sigma^{2}}} \exp \left\{-\frac{x^{2}}{2 m \sigma^{2}}\right\}\right|=0 .
$$

Use this in a Riemann sum argument (details as exercise).

For the homogeneous $\bar{q}$-walk this lemma gives (using symmetry)

$$
\lim _{n \rightarrow \infty} \frac{1}{\sqrt{n}} \sum_{k=0}^{\lfloor n t\rfloor-1} \bar{q}^{k}\left(x_{n}, 0\right)=\frac{1}{2 \sigma_{1}^{2}} \int_{0}^{2 \sigma_{1}^{2} t} \frac{1}{\sqrt{2 \pi v}} \exp \left\{-\frac{(r-q)^{2}}{2 v}\right\} d v .
$$

Now the task is to relate the transitions $q$ and $\bar{q}$. For this purpose we introduce one more player: the potential kernel of the symmetric $\bar{q}$-walk, defined by

$$
\begin{aligned}
\bar{a}(x) & =\lim _{n \rightarrow \infty}\left[\bar{G}_{n}(0,0)-\bar{G}_{n}(x, 0)\right] \\
& =\lim _{n \rightarrow \infty}\left\{\sum_{k=0}^{n} \bar{q}^{k}(0,0)-\sum_{k=0}^{n} \bar{q}^{k}(x, 0)\right\} .
\end{aligned}
$$

The potential kernel satisfies $\bar{a}(0)=0$, the equations

$$
\bar{a}(x)=\sum_{y \in \mathbb{Z}} \bar{q}(x, y) \bar{a}(y) \quad \text { for } x \neq 0, \text { and } \quad \sum_{y \in \mathbb{Z}} \bar{q}(0, y) \bar{a}(y)=1,
$$


and the limit

$$
\lim _{x \rightarrow \pm \infty} \frac{\bar{a}(x)}{|x|}=\frac{1}{2 \sigma_{1}^{2}} .
$$

(For existence of $\bar{a}$ and its properties, see [Spi76, Sections 28-29].)

Example 4.4. If for some $k \in \mathbb{Z}, p(0, k)+p(0, k+1)=1$, so that $\bar{q}(0, x)=$ 0 for $x \notin\{-1,0,1\}$, then $\bar{a}(x)=|x| /\left(2 \sigma_{a}^{2}\right)$.

Define the constant

$$
\beta=\sum_{x \in \mathbb{Z}} q(0, x) \bar{a}(x) .
$$

This constant accounts for the difference in the limits of the Green's functions for transitions $q$ and $\bar{q}$.

Lemma 4.3. Let $x \in \mathbb{R}$ and $x_{n} \in \mathbb{Z}$ be such that $x_{n}-n^{1 / 2} x$ stays bounded. Then

$$
\lim _{n \rightarrow \infty} n^{-1 / 2} G_{n}\left(x_{n}, 0\right)=\frac{1}{2 \beta \sigma_{1}^{2}} \int_{0}^{2 \sigma_{1}^{2}} \frac{1}{\sqrt{2 \pi v}} \exp \left\{-\frac{x^{2}}{2 v}\right\} d v .
$$

Proof. Given limit (4.18), it suffices to prove

$$
\sup _{z \in \mathbb{Z}}\left|\frac{\beta}{\sqrt{n}} G_{n}(z, 0)-\frac{1}{\sqrt{n}} \bar{G}_{n}(z, 0)\right| \rightarrow 0 .
$$

First we prove the case $z=0$.

By (4.18),

$$
\lim _{n \rightarrow \infty} n^{-1 / 2} \bar{G}_{n}(0,0)=\frac{1}{\sqrt{\pi \sigma_{1}^{2}}}
$$

We need to show

$$
\lim _{n \rightarrow \infty} n^{-1 / 2} G_{n}(0,0)=\frac{1}{\beta \sqrt{\pi \sigma_{1}^{2}}} .
$$

Using (4.20), $\bar{a}(0)=0$, and $\bar{q}(x, y)=q(x, y)$ for $x \neq 0$,

$$
\begin{aligned}
\sum_{x \in \mathbb{Z}} q^{m}(0, x) \bar{a}(x) & =\sum_{x \neq 0} q^{m}(0, x) \bar{a}(x)=\sum_{x \neq 0, y \in \mathbb{Z}} q^{m}(0, x) \bar{q}(x, y) \bar{a}(y) \\
& =\sum_{x \neq 0, y \in \mathbb{Z}} q^{m}(0, x) q(x, y) \bar{a}(y) \\
& =\sum_{y \in \mathbb{Z}} q^{m+1}(0, y) \bar{a}(y)-q^{m}(0,0) \sum_{y \in \mathbb{Z}} q(0, y) \bar{a}(y) .
\end{aligned}
$$

Constant $\beta$ appears in the last term. Sum over $m=0,1, \ldots, n-1$ to get

$$
\left(1+q(0,0)+\cdots+q^{n-1}(0,0)\right) \beta=\sum_{x \in \mathbb{Z}} q^{n}(0, x) \bar{a}(x)
$$


and write this in the form

$$
n^{-1 / 2} \beta G_{n-1}(0,0)=n^{-1 / 2} E_{0}\left[\bar{a}\left(Y_{n}\right)\right] .
$$

Recall that $Y_{n}=X_{n}-\tilde{X}_{n}$ where $X_{n}$ and $\tilde{X}_{n}$ are two independent walks in the same environment. By the quenched CLT for space-time RWRE, $n^{-1 / 2} Y_{n} \stackrel{\mathcal{D}}{\longrightarrow} \mathcal{N}\left(0,2 \sigma_{1}^{2}\right)$. Marginally $X_{n}$ and $\tilde{X}_{n}$ are i.i.d. walks with bounded steps, hence there is enough uniform integrability to conclude that

$$
n^{-1 / 2} E_{0}\left|Y_{n}\right| \rightarrow 2 \sqrt{\sigma_{1}^{2} / \pi}
$$

By (4.21) and some estimation (exercise),

$$
n^{-1 / 2} E_{0}\left[\bar{a}\left(Y_{n}\right)\right] \rightarrow \frac{1}{\sqrt{\sigma_{1}^{2} \pi}} .
$$

This proves (4.26) and thereby limit (4.24) for $z=0$.

To get the full statement in (4.24), for $k \geq 1$ and $z \neq 0$ let

$$
f^{k}(z, 0)=\mathbf{1}_{\{z \neq 0\}} \sum_{z_{1} \neq 0, \ldots, z_{k-1} \neq 0} q\left(z, z_{1}\right) q\left(z_{1}, z_{2}\right) \cdots q\left(z_{k-1}, 0\right)
$$

denote the probability that the first visit to the origin occurs at time $k$. This quantity is the same for both $q$ and $\bar{q}$ because these processes do not differ until the origin is visited. Choose $n_{0}$ so that

$$
\left|\beta G_{m}(0,0)-\bar{G}_{m}(0,0)\right| \leq \varepsilon \sqrt{m} \text { for } m \geq n_{0} .
$$

Then

$$
\begin{aligned}
& \sup _{z \neq 0}\left|\frac{\beta}{\sqrt{n}} G_{n}(z, 0)-\frac{1}{\sqrt{n}} \bar{G}_{n}(z, 0)\right| \\
& \leq \sup _{z \neq 0} \frac{1}{\sqrt{n}} \sum_{k=1}^{n} f^{k}(z, 0)\left|\beta G_{n-k}(0,0)-\bar{G}_{n-k}(0,0)\right| \\
& \leq \sup _{z \neq 0} \frac{\varepsilon}{\sqrt{n}} \sum_{k=1}^{n-n_{0}} f^{k}(z, 0) \sqrt{n-k}+\frac{C n_{0}^{2}}{\sqrt{n}} \leq \varepsilon+\frac{C n_{0}^{2}}{\sqrt{n}} .
\end{aligned}
$$

Letting $n \rightarrow \infty$ completes the proof.

Combining (4.17) and (4.23) gives

$$
\begin{aligned}
\lim _{n \rightarrow \infty} \mathbb{E}\left[\bar{H}_{n}(t, q) \bar{H}_{n}(t, r)\right] & =\frac{\sigma_{D}^{2}}{2 \beta \sigma_{1}^{2}} \int_{0}^{2 \sigma_{1}^{2}} \frac{1}{\sqrt{2 \pi v}} \exp \left\{-\frac{x^{2}}{2 v}\right\} d v \\
& =\kappa \Gamma_{1}((t, q),(t, r))
\end{aligned}
$$


where we defined a the new constant

$$
\kappa=\frac{\sigma_{D}^{2}}{\beta \sigma_{1}^{2}} .
$$

While we have not furnished all the details, let us consider proved that for a fixed $t$, the finite-dimensional distributions of $\bar{H}(t, r)$ converge to the Gaussian process $H(t, r)$ with covariance $\kappa \Gamma_{1}((t, q),(t, r))$.

\section{Step 2. Markov property for time steps.}

This step is overly technical and so we only give a sketch of the idea behind it. Stopping and restarting the walk $X_{\text {. }}^{\lfloor n t b\rfloor+\lfloor r \sqrt{n}\rfloor,\lfloor n t\rfloor}$ at level $\lfloor n s\rfloor$ gives:

$$
\begin{aligned}
& E^{\omega}\left(X_{\lfloor n t\rfloor}^{\lfloor n t b\rfloor+\lfloor r \sqrt{n}\rfloor,\lfloor n t\rfloor}\right)-\lfloor r \sqrt{n}\rfloor \\
& =\sum_{x \in \mathbb{Z}} P^{\omega}\left(X_{\lfloor n t\rfloor-\lfloor n s\rfloor}^{\lfloor n t b\rfloor+\lfloor r \sqrt{n}\rfloor,\lfloor n t\rfloor}=\lfloor n s b\rfloor+x\right) E^{\omega}\left(X_{\lfloor n s\rfloor}^{\lfloor n s b+x,\lfloor n s\rfloor}\right)-\lfloor r \sqrt{n}\rfloor \\
& =\sum_{x \in \mathbb{Z}} P^{\omega}\left(X_{\lfloor n t\rfloor-\lfloor n s\rfloor}^{\lfloor n t b\rfloor+\lfloor r \sqrt{n}\rfloor,\lfloor n t\rfloor}=\lfloor n s b\rfloor+x\right)\left[E^{\omega}\left(X_{\lfloor n s\rfloor}^{\lfloor n s b\rfloor+x,\lfloor n s\rfloor}\right)-x\right] \\
& \quad+E^{\omega}\left(X_{\lfloor n t\rfloor-\lfloor n s\rfloor}^{\lfloor n t b\rfloor+\lfloor r \sqrt{n}\rfloor,\lfloor n t\rfloor}\right)-\lfloor n s b\rfloor-\lfloor r \sqrt{n}\rfloor .
\end{aligned}
$$

Change summation index to $u=x / \sqrt{n}$. Then we have approximately the identity

$$
\begin{aligned}
\bar{H}_{n}(t, r) & = \\
\sum_{u \in n^{-1 / 2} \mathbb{Z}} & P^{\omega}\left\{\frac{X_{\lfloor n t\rfloor-\lfloor n s\rfloor}^{\lfloor n t b\rfloor+\lfloor r \sqrt{n}\rfloor,\lfloor n t\rfloor}-\lfloor n s b\rfloor-r \sqrt{n}}{\sqrt{n}}=u-r\right\} \bar{H}_{n}(s, u) \\
& +\bar{H}_{n}^{*}(t-s, r),
\end{aligned}
$$

where $\bar{H}_{n}^{*}(t-s, r)$ is the same as $\bar{H}_{n}(t-s, r)$ but with origin shifted (approximately) to $(n s b, n s)$. On the right-hand side, the processes $\bar{H}_{n}(s, \cdot)$ and $\bar{H}_{n}^{*}(t-s, \cdot)$ are independent of each other because they depend on disjoint levels of environments: $\bar{H}_{n}(s, \cdot)$ uses $\bar{\omega}_{1,\lfloor n s\rfloor}$ and $\bar{H}_{n}^{*}(t-s, \cdot)$ uses $\bar{\omega}_{\lfloor n s\rfloor+1,\lfloor n t\rfloor}$. As $n \rightarrow \infty$ the probability coefficients of the sum converge to deterministic Gaussian probabilities by the quenched CLT for the RWRE. By the result for fixed $t$, the right-hand side above converges in distribution.

Taking the limits and supplying all the technicalities leads to the equation

$$
H(t, r)=\int_{\mathbb{R}} \varphi_{\sigma_{1}^{2}(t-s)}(u-r) H(s, u) d u+H^{*}(t-s, r)
$$

where on the right, the processes $H(s, \cdot)$ and $H^{*}(t-s, \cdot)$ are independent. From this equation one can verify that the finite-dimensional distributions 
of the process $H(t, r)$ are Gaussian with covariance $\kappa \Gamma_{1}((s, q),(t, r))$ as stated in Theorem 4.3.

This concludes the presentation of the random average process limit.

\section{References}

The fluctuation results for RAP presented here are from [BRAS06]. In addition to [FF98], RAP was later studied also in [FMV03]. The quenched CLT for space-time RWRE has been proved several times with progressively better assumptions, see [RAS05]. 


\section{Chapter 5}

\section{Asymmetric simple exclusion process}

The asymmetric simple exclusion process (ASEP) is a Markov process that describes the motion of particles on the one-dimensional integer lattice $\mathbb{Z}$. Each particle executes a continuous-time nearest-neighbor random walk on $\mathbb{Z}$ with jump rate $p$ to the right and $q$ to the left. Particles interact through the exclusion rule which means that at most one particle is allowed at each site. Any attempt to jump onto an already occupied site is prevented from happening. The asymmetric case is $p \neq q$. We assume $0 \leq q<p \leq 1$ and $p+q=1$.

For this process we do not derive precise distributional limits for the current, but only bounds that reveal the order of magnitude of the fluctuations. In contrast with the earlier results for linear flux, the magnitude of current fluctuations is now $t^{1 / 3}$.

The proofs of these bounds are based on couplings, and make heavy use of the notion of second class particle.

\subsection{Basic properties}

We run quickly through the fundamentals of $(p, q)$-ASEP.

Definition and graphical construction. The state of the system at time $t$ is a configuration $\eta(t)=\left(\eta_{i}(t)\right)_{i \in \mathbb{Z}} \in\{0,1\}^{\mathbb{Z}}$ of zeroes and ones. The value $\eta_{i}(t)=1$ means that site $i$ is occupied by a particle at time $t$, while the value $\eta_{i}(t)=0$ means that site $i$ is vacant at time $t$.

The motion of the particles is controlled by independent Poisson processes (Poisson clocks) $\left\{N^{i \rightarrow i+1}, N^{i \rightarrow i-1}: i \in \mathbb{Z}\right\}$ on $\mathbb{R}_{+}$. These Poisson processes are independent of the (possibly random) initial configuration 
$\eta(0)$. Each Poisson clock $N^{i \rightarrow i+1}$ has rate $p$ and each $N^{i \rightarrow i-1}$ has rate $q$. If $t$ is a jump time for $N^{i \rightarrow i+1}$ and if $\left(\eta_{i}(t-), \eta_{i+1}(t-)\right)=(1,0)$ then at time $t$ the particle from site $i$ moves to site $i+1$ and the new values are $\left(\eta_{i}(t), \eta_{i+1}(t)\right)=(0,1)$. Similarly if $t$ is a jump time for $N^{i \rightarrow i-1}$ a particle is moved from $i$ to $i-1$ at time $t$, provided the configuration at time $t-$ permits this move. If the jump prompted by a Poisson clock is not permitted by the state of the system, this jump attempt is simply ignored and the particles resume waiting for the next prompt coming from the Poisson clocks.

This construction of the process is known as the graphical construction or the Harris construction. When the initial state is a fixed configuration $\eta, P^{\eta}$ denotes the distribution of the process.

We write $\eta, \omega$, etc for elements of the state space $\{0,1\}^{\mathbb{Z}}$, but also for the entire process so that $\eta$-process stands for $\left\{\eta_{i}(t): i \in \mathbb{Z}, 0 \leq t<\infty\right\}$. The configuration $\delta_{i}$ is the state that has a single particle at position $i$ but otherwise the lattice is vacant.

Remark 5.1. When infinite particle systems such as ASEP are constructed with Poisson clocks, there is an issue of well-definedness that needs to be resolved. Namely, if we ask whether site $x$ is occupied at time $t$, we need to look backwards in time at all the possible sites from which a particle could have moved to $x$ by time $t$. This might involve an infinite regression: perhaps there is a sequence of times $t>t_{1}>t_{2}>t_{3}>\cdots>0$ such that Poisson clock $N^{x-k \rightarrow x-k+1}$ signaled a jump attempt at time $t_{k}$. Such a sequence of jumps could in principle bring a particle to $x$ "from infinity."

However, it is easily seen that this happens only with probability zero. For any fixed $T<\infty$ there is a positive probability that both $N^{i \rightarrow i+1}$ and $N^{i+1 \rightarrow i}$ are empty in $[0, T]$. Consequently almost surely there are infinitely many edges $(i, i+1)$ across which no jump attempts are made before time $T$. This way the construction can actually be performed for finite portions of the lattice at a time.

Similar issue arises with the possibility of simultaneous conflicting jump commands. By excluding a zero-probability set of realizations of $\left\{N^{i \rightarrow i \pm 1}\right\}$ we can assume that there are no simultaneous jump attempts.

Invariant distributions. A basic fact is that i.i.d. Bernoulli distributions $\left\{\nu^{\rho}\right\}_{\rho \in[0,1]}$ are extremal invariant distributions for ASEP. For each density value $\rho \in[0,1], \nu^{\rho}$ is the probability measure on $\{0,1\}^{\mathbb{Z}}$ under which the occupation variables $\left\{\eta_{i}\right\}$ are i.i.d. with common mean $\int \eta_{i} d \nu^{\rho}=\rho$. When the process $\eta$ is stationary with time-marginal $\nu^{\rho}$, we write $P^{\rho}$ for the probability distribution of the entire process. The stationary density- $\rho$ process means the ASEP $\eta$ that is stationary in time and has marginal distribution $\eta(t) \sim \nu_{\rho}$ for all $t \in \mathbb{R}_{+}$.

Remark 5.2. A note about how one would check the invariance. In general, the infinitesimal generator $L$ of a Markov process is an operator defined 
as the derivative of the semigroup:

$$
L \varphi(\eta)=\lim _{t \searrow 0} \frac{E^{\eta}[f(\eta(t))]-f(\eta)}{t} .
$$

Above $E^{\eta}$ denotes expectation under $P^{\eta}$, the distribution of the process when the initial state is $\eta$. The generator of ASEP is

$$
\begin{aligned}
L \varphi(\eta)=p & \sum_{i \in \mathbb{Z}} \eta_{i}\left(1-\eta_{i+1}\right)\left[\varphi\left(\eta^{i, i+1}\right)-\varphi(\eta)\right] \\
& +q \sum_{i \in \mathbb{Z}} \eta_{i}\left(1-\eta_{i-1}\right)\left[\varphi\left(\eta^{i, i-1}\right)-\varphi(\eta)\right]
\end{aligned}
$$

that acts on cylinder functions $\varphi$ on the state space $\{0,1\}^{\mathbb{Z}}$ and $\eta^{i, j}=$ $\eta-\delta_{i}+\delta_{j}$ is the configuration that results from moving one particle from site $i$ to $j$. Equation (5.1) can be derived from the graphical construction with some estimation.

Invariance of a probability distribution can be checked by a generator computation. For ASEP it is enough to check that

$$
\int L \varphi d \mu=0
$$

for cylinder functions $\varphi$ to conclude that $\mu$ is invariant. This can be used to check that Bernoulli measures $\nu^{\rho}$ are invariant for ASEP.

Basic coupling and second class particles. The basic coupling of two exclusion processes $\eta$ and $\omega$ means that they obey a common set of Poisson clocks $\left\{N^{i \rightarrow i+1}, N^{i \rightarrow i-1}\right\}$. Suppose the two processes $\eta$ and $\eta^{+}$satisfy $\eta^{+}(0)=\eta(0)+\delta_{Q(0)}$ at time zero, for some position $Q(0) \in$ $\mathbb{Z}$. This means that $\eta_{i}^{+}(0)=\eta_{i}(0)$ for all $i \neq Q(0), \eta_{Q(0)}^{+}(0)=1$ and $\eta_{Q(0)}(0)=0$. Then throughout the evolution in the basic coupling there is a single discrepancy between $\eta(t)$ and $\eta^{+}(t)$ at some position $Q(t): \eta^{+}(t)=$ $\eta(t)+\delta_{Q(t)}$. From the perspective of $\eta(t), Q(t)$ is called a second class particle. By the same token, from the perspective of $\eta^{+}(t), Q(t)$ is a second class antiparticle. In particular, we shall call the pair $(\eta, Q)$ a $(p, q)-A S E P$ with a second class particle.

We write a boldface $\mathbf{P}$ for the probability measure when more than one process are coupled together. In particular, $\mathbf{P}^{\rho}$ represents the situation where the initial occupation variables $\eta_{i}(0)=\eta_{i}^{+}(0)$ are i.i.d. mean- $\rho$ Bernoulli for $i \neq 0$, and the second class particle $Q$ starts at $Q(0)=0$.

More generally, if two processes $\eta$ and $\omega$ are in basic coupling and $\omega(0) \geq$ $\eta(0)$ (by which we mean coordinatewise ordering $\omega_{i}(0) \geq \eta_{i}(0)$ for all $i$ ) then the ordering $\omega(t) \geq \eta(t)$ holds for all $0 \leq t<\infty$. The effect of the basic coupling is to give priority to the $\eta$ particles over the $\omega-\eta$ particles. 
Consequently we can think of the $\omega$-process as consisting of first class particles (the $\eta$ particles) and second class particles (the $\omega-\eta$ particles).

Current. For $x \in \mathbb{Z}$ and $t>0, J_{x}(t)$ stands for the net left-to-right particle current across the straight-line space-time path from $(1 / 2,0)$ to $(x+1 / 2, t)$. More precisely, $J_{x}(t)=J_{x}(t)^{+}-J_{x}(t)^{-}$where $J_{x}(t)^{+}$is the number of particles that lie in $(-\infty, 0]$ at time 0 but lie in $[x+1, \infty)$ at time $t$, while $J_{x}(t)^{-}$is the number of particles that lie in $[1, \infty)$ at time 0 and in $(-\infty, x]$ at time $t$. When more than one process $(\omega, \eta$, etc $)$ is considered in a coupling, the currents of the processes are denoted by $J_{x}^{\omega}(t), J_{x}^{\eta}(t)$, etc.

\subsection{Results}

The average net rate at which particles in the stationary $(p, q)$-ASEP at density $\rho$ move across a fixed edge $(i, i+1)$ is the flux

$$
H(\rho)=E^{\rho}\left[J_{0}(t)\right]=(p-q) \rho(1-\rho) .
$$

This formula follows from the fact that this process $M(t)$ is a mean zero martingale:

$$
\begin{aligned}
& M(t)=J_{0}(t)-\int_{0}^{t}(p \mathbf{1}\{\left.\eta_{0}(s)=1, \eta_{1}(s)=0\right\} \\
&\left.-q \mathbf{1}\left\{\eta_{1}(s)=1, \eta_{0}(s)=0\right\}\right) d s .
\end{aligned}
$$

For the more general currents

$$
E^{\rho}\left[J_{x}(t)\right]=t H(\rho)-x \rho \quad(x \in \mathbb{Z}, t \geq 0)
$$

as can be seen by noting that particles that crossed the edge $(0,1)$ either also crossed $(x, x+1)$ and contributed to $J_{x}(t)$ or did not.

The characteristic speed at density $\rho$ is

$$
V^{\rho}=H^{\prime}(\rho)=(p-q)(1-2 \rho) .
$$

The derivation of the fluctuation bounds for the current rests on several key identities which we collect in the next theorem.

Theorem 5.3. Let the second class particle start at the origin: $Q(0)=0$. For any density $0<\rho<1, z \in \mathbb{Z}$ and $t>0$ we have these formulas.

$$
\begin{gathered}
\operatorname{Var}^{\rho}\left[J_{z}(t)\right]=\sum_{j \in \mathbb{Z}}|j-z| \operatorname{Cov}^{\rho}\left[\eta_{j}(t), \eta_{0}(0)\right], \\
\operatorname{Cov}^{\rho}\left[\eta_{j}(t), \eta_{0}(0)\right]=\rho(1-\rho) \mathbf{P}^{\rho}\{Q(t)=j\},
\end{gathered}
$$


and

$$
\mathbf{E}^{\rho}[Q(t)]=V^{\rho} t
$$

Formulas (5.8) and (5.9) combine to give

$$
\operatorname{Var}^{\rho}\left[J_{z}(t)\right]=\rho(1-\rho) \mathbf{E}^{\rho}|Q(t)-z| .
$$

In particular, for the current across the characteristic,

$$
\operatorname{Var}^{\rho}\left[J_{\left\lfloor V^{\rho} t\right\rfloor}(t)\right]=\rho(1-\rho) \mathbf{E}^{\rho}\left|Q(t)-\left\lfloor V^{\rho} t\right\rfloor\right| .
$$

Thus to get variance bounds on the current, we derive moment bounds on the second class particle.

We now state the main result, the moment bounds on the second class particle. It is of interest to see how the bounds depend on the bias $\theta=p-q$ so we include that in the estimates.

Theorem 5.4. There exist constants $0<c_{0}, C<\infty$ such that, for all $0<\theta<1 / 2,0<\rho<1,1 \leq m<3$, and $t \geq c_{0} \theta^{-4}$,

$$
\frac{1}{C} \theta^{2 m / 3} t^{2 m / 3} \leq \mathbf{E}^{\rho}\left[\left|Q(t)-V^{\rho} t\right|^{m}\right] \leq \frac{C}{3-m} \theta^{2 m / 3} t^{2 m / 3} .
$$

For the upper bound the constants are fixed for all values of the parameters. For the lower bound both constants $c_{0}, C$ depend on the density $\rho$.

As a corollary for $m=1$, we obtain the bounds for the variance of the current seen by an observer traveling at the characteristic speed $V^{\rho}$ : for $t \geq c_{0}(\rho) \theta^{-4}$,

$$
C_{1}(\rho) \theta^{1 / 3} t^{2 / 3} \leq \operatorname{Var}^{\rho}\left[J_{\left\lfloor V^{\rho} t\right\rfloor}(t)\right] \leq C_{2} \theta^{1 / 3} t^{2 / 3} .
$$

It follows from the variance bound (5.14) that for $v \neq V^{\rho}$ a Gaussian limit in the central limit scale holds:

$$
\frac{J_{[t v]}(t)-E^{\rho}\left(J_{[t v]}(t)\right)}{t^{1 / 2}} \stackrel{\mathcal{D}}{\longrightarrow} \chi
$$

for a centered normal random variable $\chi$. To observe this, take the case $v>V^{\rho}$. Let $J^{*}$ be the current across the straight-line space-time path from $\left(\left(v-V^{\rho}\right) t, 0\right)$ to $(v t, t)$. This current has variance of order $t^{2 / 3}$. Then use

$$
J^{*}=J_{[t v]}(t)+\sum_{i=1}^{\left(v-V^{\rho}\right) t} \eta_{i}(0) .
$$

A distributional limit exists for the current for the case of the stationary totally asymmetric simple exclusion process (TASEP). We state the result here. In TASEP particles march only to the right (say), and so $p=1$ and $q=0$. 
Theorem 5.5. [FS06] In stationary TASEP, the following distributional convergence holds:

$$
\lim _{t \rightarrow \infty} P^{\rho}\left\{\frac{J_{\left\lfloor V^{\rho} t\right\rfloor}(t)-\rho^{2} t}{\rho^{2 / 3}(1-\rho)^{2 / 3} t^{1 / 3}} \leq x\right\}=F_{0}(x)
$$

The distribution function $F_{0}$ above is defined in [FS06] as $F_{0}(x)=$ $(\partial / \partial x)\left(F_{\mathrm{GUE}}(x) g(x, 0)\right)$ where $F_{\mathrm{GUE}}$ is the Tracy-Widom GUE distribution and $g$ a certain scaling function.

Theorem 5.5 will not be discussed further, and we turn to proofs of Theorem 5.3 and Theorem 5.4. In the next section we give partial proofs of the identities in Theorem 5.3. Section 5.4 describes a coupling that we use to control second class particles, and a random walk bound that comes in handy. The last two sections of this chapter prove the upper and lower bounds of Theorem 5.4.

\subsection{Proofs for the identities}

Let $\omega$ be a stationary exclusion process with i.i.d. Bernoulli $(\rho)$ distributed occupations $\left\{\omega_{i}(t)\right\}$ at any fixed time $t$.

Proof of equation (5.8). This is partly a hand-waiving proof. What is missing is justification for certain limits.

To approximate the infinite system with finite systems, for each $N \in \mathbb{N}$ let process $\omega^{N}$ have initial configuration

$$
\omega_{i}^{N}(0)=\omega_{i}(0) \mathbf{1}_{\{-N \leq i \leq N\}} .
$$

We assume that all these processes are coupled through common Poisson clocks. Let $J_{z}^{N}(t)$ denote the current in process $\omega^{N}$.

Let $z(0)=0, z(t)=z$, and introduce the counting variables

$$
I_{+}^{N}(t)=\sum_{n>z(t)} \omega_{n}^{N}(t), \quad I_{-}^{N}(t)=\sum_{n \leq z(t)} \omega_{n}^{N}(t) .
$$

Then the current can be expressed as

$$
J_{z}^{N}(t)=I_{+}^{N}(t)-I_{+}^{N}(0)=I_{-}^{N}(0)-I_{-}^{N}(t),
$$

and its variance as

$$
\begin{aligned}
\operatorname{Var} J_{z}^{N}(t)= & \operatorname{Cov}\left(I_{+}^{N}(t)-I_{+}^{N}(0), I_{-}^{N}(0)-I_{-}^{N}(t)\right) \\
= & \operatorname{Cov}\left(I_{+}^{N}(t), I_{-}^{N}(0)\right)+\operatorname{Cov}\left(I_{+}^{N}(0), I_{-}^{N}(t)\right) \\
& -\operatorname{Cov}\left(I_{+}^{N}(0), I_{-}^{N}(0)\right)-\operatorname{Cov}\left(I_{+}^{N}(t), I_{-}^{N}(t)\right) .
\end{aligned}
$$


Independence of initial occupation variables gives

$$
\operatorname{Cov}\left(I_{+}^{N}(0), I_{-}^{N}(0)\right)=0
$$

and the identity above simplifies to

$$
\begin{aligned}
& \operatorname{Var} J_{z}^{N}(t)=\operatorname{Cov}\left(I_{+}^{N}(t), I_{-}^{N}(0)\right)+\operatorname{Cov}\left(I_{+}^{N}(0), I_{-}^{N}(t)\right) \\
& -\operatorname{Cov}\left(I_{+}^{N}(t), I_{-}^{N}(t)\right) \\
& =\sum_{k \leq 0, m>z} \operatorname{Cov}\left[\omega_{m}^{N}(t), \omega_{k}^{N}(0)\right] \\
& \quad+\sum_{k \leq z, m>0} \operatorname{Cov}\left[\omega_{k}^{N}(t), \omega_{m}^{N}(0)\right]-\operatorname{Cov}\left(I_{+}^{N}(t), I_{-}^{N}(t)\right) .
\end{aligned}
$$

In the $N \rightarrow \infty$ limit variables $\omega_{i}^{N}(t)$ converge (a.s. and in $L^{2}$ ) to the i.i.d. occupation variables $\omega_{i}(t)$ of the stationary process. It follows from the graphical construction that on a fixed time interval covariances can be bounded exponentially, uniformly over $N$ : for a fixed $0<t<\infty$,

$$
\left|\operatorname{Cov}\left[\omega_{m}^{N}(t), \omega_{k}^{N}(s)\right]\right| \leq C e^{-c_{1}|m-k|} \text { for } s \in[0, t] .
$$

Hence in the limit the last covariance in (5.19) vanishes. Furthermore, $J_{z}^{N}(t) \rightarrow J_{z}(t)$ similarly, so in the limit we get

$$
\begin{aligned}
\operatorname{Var} J_{z}(t) & =\sum_{k \leq 0, m>z} \operatorname{Cov}\left[\omega_{m}(t), \omega_{k}(0)\right]+\sum_{k \leq z, m>0} \operatorname{Cov}\left[\omega_{k}(t), \omega_{m}(0)\right] \\
& =\sum_{n \in \mathbb{Z}}|n-z| \operatorname{Cov}\left[\omega_{n}(t), \omega_{0}(0)\right]
\end{aligned}
$$

This proves equation (5.8).

Proof of equation (5.9). This is a straight-forward calculation.

$$
\begin{aligned}
& \operatorname{Cov}^{\rho}\left[\omega_{j}(t), \omega_{0}(0)\right]=E^{\rho}\left[\omega_{j}(t) \omega_{0}(0)\right]-\rho^{2} \\
& =\rho E^{\rho}\left[\omega_{j}(t) \mid \omega_{0}(0)=1\right]-\rho E^{\rho}\left[\omega_{j}(t)\right] \\
& =\rho\left(E^{\rho}\left[\omega_{j}(t) \mid \omega_{0}(0)=1\right]-\rho E^{\rho}\left[\omega_{j}(t) \mid \omega_{0}(0)=1\right]\right. \\
& \left.\quad-(1-\rho) E^{\rho}\left[\omega_{j}(t) \mid \omega_{0}(0)=0\right]\right) \\
& =\rho(1-\rho)\left(E^{\rho}\left[\omega_{j}(t) \mid \omega_{0}(0)=1\right]-E^{\rho}\left[\omega_{j}(t) \mid \omega_{0}(0)=0\right]\right) \\
& =\rho(1-\rho)\left(\mathbf{E}^{\rho}\left[\omega_{j}^{+}(t)\right]-\mathbf{E}^{\rho}\left[\omega_{j}(t)\right]\right)=\rho(1-\rho) \mathbf{E}^{\rho}\left[\omega_{j}^{+}(t)-\omega_{j}(t)\right] \\
& =\rho(1-\rho) \mathbf{P}^{\rho}[Q(t)=j] .
\end{aligned}
$$


Proof of equation (5.10). Let again $\omega^{N}$ be the finite process with initial condition (5.17). Let $I^{N}=\sum_{i} \omega_{i}^{N}(t)$ be the number of particles in the process $\omega^{N} \cdot I^{N}$ is a $\operatorname{Binomial}(2 N+1, \rho)$ random variable. For $0<\rho<1$

$$
\begin{aligned}
& \frac{d}{d \rho} E\left[J_{z}^{N}(t)\right]=\frac{d}{d \rho} \sum_{m=0}^{2 N+1}\left(\begin{array}{c}
2 N+1 \\
m
\end{array}\right) \rho^{m}(1-\rho)^{2 N+1-m} E\left[J_{z}^{N}(t) \mid I^{N}=m\right] \\
& =\sum_{m=0}^{2 N+1} P\left(I^{N}=m\right)\left(\frac{m}{\rho}-\frac{2 N+1-m}{1-\rho}\right) E\left[J_{z}^{N}(t) \mid I^{N}=m\right] \\
& =\frac{1}{\rho(1-\rho)} E\left[J_{z}^{N}(t)\left(I^{N}-(2 N+1) \rho\right)\right] \\
& =\frac{1}{\rho(1-\rho)} \operatorname{Cov}\left[I_{+}^{N}(t)-I_{+}^{N}(0), I_{-}^{N}(0)+I_{+}^{N}(0)\right] \\
& =\frac{1}{\rho(1-\rho)}\left(\operatorname{Cov}\left[I_{+}^{N}(t), I_{-}^{N}(0)\right]+\operatorname{Cov}\left[I_{+}^{N}(t)-I_{+}^{N}(0), I_{+}^{N}(0)\right]\right) .
\end{aligned}
$$

The last equality used $\operatorname{Cov}\left[I_{+}^{N}(0), I_{-}^{N}(0)\right]=0$ that comes from the i.i.d. distribution of initial occupations. The first covariance on line (5.20) write directly as

$$
\operatorname{Cov}\left[I_{+}^{N}(t), I_{-}^{N}(0)\right]=\sum_{k \leq 0, m>z} \operatorname{Cov}\left[\omega_{m}^{N}(t), \omega_{k}^{N}(0)\right] .
$$

The second covariance on line (5.20) write as

$$
\begin{aligned}
\operatorname{Cov}\left[I_{+}^{N}(t)-I_{+}^{N}(0), I_{+}^{N}(0)\right]=\operatorname{Cov}\left[I_{-}^{N}(0)-I_{-}^{N}(t), I_{+}^{N}(0)\right] \\
\quad=-\operatorname{Cov}\left[I_{-}^{N}(t), I_{+}^{N}(0)\right]=-\sum_{k \leq z, m>0} \operatorname{Cov}\left[\omega_{k}^{N}(t), \omega_{m}^{N}(0)\right] .
\end{aligned}
$$

Inserting these back on line (5.20) gives

$$
\begin{aligned}
\frac{d}{d \rho} E\left[J_{z}^{N}(t)\right]= & \frac{1}{\rho(1-\rho)}\left(\sum_{k \leq 0, m>z} \operatorname{Cov}\left[\omega_{m}^{N}(t), \omega_{k}^{N}(0)\right]\right. \\
& \left.-\sum_{k \leq z, m>0} \operatorname{Cov}\left[\omega_{k}^{N}(t), \omega_{m}^{N}(0)\right]\right)
\end{aligned}
$$

Compared to line (5.19) we have the difference instead of the sum. Integrate over the density $\rho$ and take $N \rightarrow \infty$, as was taken in (5.19), to obtain

$$
\begin{aligned}
E^{\rho}\left[J_{z}(t)\right]-E^{\lambda}\left[J_{z}(t)\right] & =\int_{\lambda}^{\rho} \frac{1}{\theta(1-\theta)} \sum_{j \in \mathbb{Z}}(j-z) \operatorname{Cov}^{\theta}\left[\omega_{j}(t), \omega_{0}(0)\right] d \theta \\
& =\int_{\lambda}^{\rho}\left(\mathbf{E}^{\theta}[Q(t)]-z\right) d \theta
\end{aligned}
$$


for $0<\lambda<\rho<1$. Couplings show the continuity of these expectations:

$$
E^{\lambda}\left[J_{z}(t)\right] \rightarrow E^{\rho}\left[J_{z}(t)\right] \quad \text { and } \quad \mathbf{E}^{\lambda}[Q(t)] \rightarrow \mathbf{E}^{\rho}[Q(t)]
$$

as $\lambda \rightarrow \rho$ in $(0,1)$. Thus the identity above can be differentiated in $\rho$. With $z=0$ and via (5.4) identity (5.10) follows.

\subsection{A coupling and a random walk bound}

As observed in (5.10) the mean speed of the second class particle in a density- $\rho$ ASEP is $H^{\prime}(\rho)$. Thus by the concavity of $H$ a defect travels on average slower in a denser system (recall that we assume $p>q$ throughout). However, the basic coupling does not respect this, except in the totally asymmetric $(p=1, q=0)$ case. To see this, consider two pairs of processes $\left(\omega^{+}, \omega\right)$ and $\left(\eta^{+}, \eta\right)$ such that both pairs have one discrepancy: $\omega^{+}(t)=\omega(t)+\delta_{Q^{\omega}(t)}$ and $\eta^{+}(t)=\eta(t)+\delta_{Q^{\eta}(t)}$. Assume that $\omega(t) \geq \eta(t)$. In basic coupling the jump from state

$$
\left[\begin{array}{cc}
\omega_{i}^{+} & \omega_{i+1}^{+} \\
\omega_{i} & \omega_{i+1} \\
\eta_{i}^{+} & \eta_{i+1}^{+} \\
\eta_{i} & \eta_{i+1}
\end{array}\right]=\left[\begin{array}{cc}
1 & 1 \\
0 & 1 \\
1 & 0 \\
0 & 0
\end{array}\right] \text { to state }\left[\begin{array}{ll}
1 & 1 \\
1 & 0 \\
1 & 0 \\
0 & 0
\end{array}\right]
$$

happens at rate $q$ and results in $Q^{\omega}=i+1>i=Q^{\eta}$.

In this section we construct a different coupling that combines the basic coupling with auxiliary clocks for second class particles. The idea is to think of a single "special" second class particle as performing a random walk on the process of $\omega-\eta$ second class particles. This coupling preserves the expected ordering of the special second class particles, hence it can be regarded as a form of microscopic concavity.

This theorem summarizes the outcome.

Theorem 5.6. Assume given two initial configurations $\left\{\zeta_{i}(0)\right\}$ and $\left\{\xi_{i}(0)\right\}$ and two not necessarily distinct positions $Q^{\zeta}(0)$ and $Q^{\xi}(0)$ on $\mathbb{Z}$. Suppose the coordinatewise ordering $\zeta(0) \geq \xi(0)$ holds, $Q^{\zeta}(0) \leq Q^{\xi}(0)$, and $\zeta_{i}(0)=\xi_{i}(0)+1$ for $i \in\left\{Q^{\zeta}(0), Q^{\xi}(0)\right\}$. Define the configuration $\zeta^{-}(0)=$ $\zeta(0)-\delta_{Q^{\zeta}(0)}$.

Then there exists a coupling of processes

$$
\left(\zeta^{-}(t), Q^{\zeta}(t), \xi(t), Q^{\xi}(t)\right)_{t \geq 0}
$$

with initial state $\left(\zeta^{-}(0), Q^{\zeta}(0), \xi(0), Q^{\xi}(0)\right)$ as described in the previous paragraph, such that both pairs $\left(\zeta^{-}, Q^{\zeta}\right)$ and $\left(\xi, Q^{\xi}\right)$ are $(p, q)$-ASEP's with a second class particle, and $Q^{\zeta}(t) \leq Q^{\xi}(t)$ for all $t \geq 0$. 
To begin the construction, put two exclusion processes $\zeta$ and $\xi$ in basic coupling, obeying Poisson clocks $\left\{N^{i \rightarrow i \pm 1}\right\}$. They are ordered so that $\zeta \geq \xi$. The $\zeta-\xi$ second class particles are labeled in increasing order $\cdots<X_{m-1}(t)<X_{m}(t)<X_{m+1}(t)<\cdots$. We assume there is at least one such second class particle, but beyond that we make no assumption about their number. Thus there is some finite or infinite subinterval $I \subseteq \mathbb{Z}$ of indices such that the positions of the $\zeta-\xi$ second class particles are given by $\left\{X_{m}(t): m \in I\right\}$.

We introduce two dynamically evolving labels $a(t), b(t) \in I$ in such a manner that $X_{a(t)}(t)$ is the position of a second class antiparticle in the $\zeta$-process, $X_{b(t)}(t)$ is the position of a second class particle in the $\xi$-process, and the ordering

$$
X_{a(t)}(t) \leq X_{b(t)}(t)
$$

is preserved by the dynamics.

The labels $a(t), b(t)$ are allowed to jump from $m$ to $m \pm 1$ only when particle $X_{m \pm 1}$ is adjacent to $X_{m}$. The labels do not take jump commands from the Poisson clocks $\left\{N^{i \rightarrow i \pm 1}\right\}$ that govern $(\xi, \zeta)$. Instead, the directed edges $(i, i+1)$ and $(i, i-1)$ are given another collection of independent Poisson clocks so that the following jump rates are realized.

(i) If $a=b$ and $X_{a+1}=X_{a}+1$ then

$$
(a, b) \text { jumps to } \begin{cases}(a, b+1) & \text { with rate } p-q \\ (a+1, b+1) & \text { with rate } q .\end{cases}
$$

(ii) If $a=b$ and $X_{a-1}=X_{a}-1$ then

$$
(a, b) \text { jumps to } \begin{cases}(a-1, b) & \text { with rate } p-q \\ (a-1, b-1) & \text { with rate } q .\end{cases}
$$

(iii) If $a \neq b$ then $a$ and $b$ jump independently with these rates:

$$
\begin{aligned}
& a \text { jumps to } \begin{cases}a+1 & \text { with rate } q \text { if } X_{a+1}=X_{a}+1 \\
a-1 & \text { with rate } p \text { if } X_{a-1}=X_{a}-1\end{cases} \\
& b \text { jumps to } \begin{cases}b+1 & \text { with rate } p \text { if } X_{b+1}=X_{b}+1 \\
b-1 & \text { with rate } q \text { if } X_{b-1}=X_{b}-1\end{cases}
\end{aligned}
$$

Let us emphasize that the pair process $(\xi, \zeta)$ is still governed by the old clocks $\left\{N^{i \rightarrow i \pm 1}\right\}$ in the basic coupling. The new clocks on edge $\{i, i+1\}$ that realize rules (i)-(iii) are not observed except when sites $\{i, i+1\}$ are both occupied by $X$-particles and at least one of $X_{a}$ or $X_{b}$ lies in $\{i, i+1\}$.

First note that if initially $a(0) \leq b(0)$ then jumps (i)-(iii) preserve the inequality $a(t) \leq b(t)$ which gives $(5.22)$. (Since the jumps in point (iii) 
happen independently, there cannot be two simultaneous jumps. So it is not possible for $a$ and $b$ to cross each other with a $(a, b) \rightarrow(a+1, b-1)$ move.)

Define processes $\zeta^{-}(t)=\zeta(t)-\delta_{X_{a(t)}(t)}$ and $\xi^{+}(t)=\xi(t)+\delta_{X_{b(t)}(t)}$. In other words, to produce $\zeta^{-}$remove particle $X_{a}$ from $\zeta$, and to produce $\xi^{+}$ add particle $X_{b}$ to $\xi$. The second key point is that, even though these new processes are no longer defined by the standard graphical construction, distributionwise they are still ASEP's with second class particles. We argue this point for $\left(\zeta^{-}, X_{a}\right)$ and leave the argument for $\left(\xi, X_{b}\right)$ to the reader.

Lemma 5.1. The pair $\left(\zeta^{-}, X_{a}\right)$ is a $(p, q)$-ASEP with a second class particle.

Proof. We check that the jump rates for the process $\left(\zeta^{-}, X_{a}\right)$, produced by the combined effect of the basic coupling with clocks $\left\{N^{i \rightarrow i \pm 1}\right\}$ and the new clocks, are the same jump rates that result from defining an (ASEP, second class particle) pair in terms of the graphical construction.

To have notation for the possible jumps, let 0 denote an empty site, 1 a $\zeta^{-}$-particle, and 2 particle $X_{a}$. Consider a fixed pair $(i, i+1)$ of sites and write $x y$ with $x, y \in\{0,1,2$,$\} for the contents of sites (i, i+1)$ before and after the jump. Then here are the possible moves across the edge $\{i, i+1\}$, and the rates that these moves would have in the basic coupling.

$$
\begin{array}{cl}
\text { Type } 1 & 10 \longrightarrow 01 \text { with rate } p \\
& 01 \longrightarrow 10 \text { with rate } q \\
\text { Type 2 } & 20 \longrightarrow 02 \text { with rate } p \\
& 02 \longrightarrow 20 \text { with rate } q \\
\text { Type } 3 & 12 \longrightarrow 21 \text { with rate } p \\
& 21 \longrightarrow 12 \text { with rate } q
\end{array}
$$

Our task is to check that the construction of $\left(\zeta^{-}, X_{a}\right)$ actually realizes these rates.

Jumps of types 1 and 2 are prompted by the clocks $\left\{N^{i \rightarrow i \pm 1}\right\}$ of the graphical construction of $(\xi, \zeta)$, and hence have the correct rates listed above.

Jumps of type 3 occur in two distinct ways.

(Type 3.1) First there can be a $\xi$-particle next to $X_{a}$, and then the rates shown above are again realized by the clocks $\left\{N^{i \rightarrow i \pm 1}\right\}$ because in the basic coupling the $\xi$-particles have priority over the $X$-particles. 
(Type 3.2) The other alternative is that both sites $\{i, i+1\}$ are occupied by $X$-particles and one of them is $X_{a}$. The clocks $\left\{N^{i \rightarrow i \pm 1}\right\}$ cannot interchange the $X$-particles across the edge $\{i, i+1\}$ because in the $(\xi, \zeta)$ graphical construction these are lower priority $\zeta$-particles that do not jump on top of each other. The otherwise missing jumps are now supplied by the "new" clocks that govern the jumps described in rules (i)-(iii).

Combining (i)-(iii) we can read that if $X_{a}=i+1$ and $X_{a-1}=i$, then $a$ jumps to $a-1$ with rate $p$. This is the first case of type 3 jumps above, corresponding to a $\zeta^{-}$-particle moving from $i$ to $i+1$ with rate $p$, and the second class particle $X_{a}$ yielding. On the other hand, if $X_{a}=i$ and $X_{a+1}=i+1$ then $a$ jumps to $a+1$ with rate $q$. This is the second case in type 3 , corresponding to a $\zeta^{-}$-particle moving from $i+1$ to $i$ with rate $q$ and exchanging places with the second class particle $X_{a}$.

We have verified that the process $\left(\zeta^{-}, X_{a}\right)$ operates with the correct rates.

To argue from the rates to the correct distribution of the process, we can make use of the process $\left(\zeta^{-}, \zeta\right)$. The processes $\left(\zeta^{-}, X_{a}\right)$ and $\left(\zeta^{-}, \zeta\right)$ determine each other uniquely. The virtue of $\left(\zeta^{-}, \zeta\right)$ is that it has a compact state space and only nearest-neighbor jumps with bounded rates. Hence by the basic theory of semigroups and generators of particle systems as developed in [Lig85], given the initial configuration, the distribution of the process is uniquely determined by the action of the generator on local functions. Thus it suffices to check that individual jumps have the correct rates across each edge $\{i, i+1\}$. This is exactly what we did above in the language of $\left(\zeta^{-}, X_{a}\right)$.

Similar argument shows that $\left(\xi, X_{b}\right)$ is a $(p, q)$-ASEP with a second class particle. To prove Theorem 5.6 take $Q^{\zeta}=X_{a}$ and $Q^{\xi}=X_{b}$. This gives the coupling whose existence is claimed in the theorem.

To conclude, let us observe that the four processes $\left(\xi, \xi^{+}, \zeta^{-}, \zeta\right)$ are not in basic coupling. For example, the jump from state

$$
\left[\begin{array}{cc}
\zeta_{i} & \zeta_{i+1} \\
\zeta_{i}^{-} & \zeta_{i+1}^{-} \\
\xi_{i}^{+} & \xi_{i+1}^{+} \\
\xi_{i} & \xi_{i+1}
\end{array}\right]=\left[\begin{array}{ll}
1 & 1 \\
0 & 1 \\
1 & 0 \\
0 & 0
\end{array}\right] \quad \text { to state }\left[\begin{array}{ll}
1 & 1 \\
1 & 0 \\
0 & 1 \\
0 & 0
\end{array}\right]
$$

happens at rate $q$ (second case of rule (i)), while in basic coupling this move is impossible.

As the second point of this section we prove a random walk estimate. Let $Z(t)$ be a continuous-time nearest-neighbor random walk on statespace $S \subseteq \mathbb{Z}$ that contains $\mathbb{Z}_{-}=\{\ldots,-2,-1,0\}$. Initially $Z(0)=0 . Z$ attempts to jump from $x$ to $x+1$ with rate $p$ for $x \leq-1$, and from $x$ to 
$x-1$ with rate $q$ for $x \leq 0$. Assume $p>q=1-p$ and let $\theta=p-q$. The rates on $S \backslash \mathbb{Z}_{-}$need not be specified.

Whether jumps are permitted or not is determined by a fixed environment expressed in terms of $\{0,1\}$-valued functions $\{u(x, t): x \in S, 0 \leq$ $t<\infty\}$. A jump across edge $\{x-1, x\}$ in either direction is permitted at time $t$ if $u(x, t)=1$, otherwise not. In other words, $u(x, t)$ is the indicator of the event that edge $\{x-1, x\}$ is open at time $t$.

Assumption. Assume that for all $x \in S$ and $T<\infty, u(x, t)$ flips between 0 and 1 only finitely many times during $0 \leq t \leq T$. Assume for convenience right-continuity: $u(x, t+)=u(x, t)$.

Lemma 5.2. For all $t \geq 0$ and $k \geq 0$,

$$
P\{Z(t) \leq-k\} \leq e^{-2 \theta k} .
$$

This bound holds for any fixed environment $\{u(x, t)\}$ subject to the assumption above.

Proof. Let $Y(t)$ be a walk that operates exactly as $Z(t)$ on $\mathbb{Z}_{-}$but is restricted to remain in $\mathbb{Z}_{-}$by setting the rate of jumping from 0 to 1 to zero. Give $Y(t)$ geometric initial distribution

$$
P\{Y(0)=-j\}=\pi(j) \equiv\left(1-\frac{q}{p}\right)\left(\frac{q}{p}\right)^{j} \quad \text { for } j \geq 0 .
$$

The initial points satisfy $Y(0) \leq Z(0)$ a.s. Couple the walks through Poisson clocks so that the inequality $Y(t) \leq Z(t)$ is preserved for all time $0 \leq t<\infty$.

Without the inhomogeneous environment $Y(t)$ would be a stationary, reversible birth and death process. We argue that even with the environment the time marginals $Y(t)$ still have distribution $\pi$. This suffices for the conclusion, for then

$$
P\{Z(t) \leq-k\} \leq P\{Y(t) \leq-k\}=(q / p)^{k}=\exp \left(k \log \frac{1-\theta}{1+\theta}\right) \leq e^{-2 \theta k} .
$$

To justify the claim about $Y(t)$, consider approximating processes $Y^{(m)}(t), m \in \mathbb{N}$, with the same initial value $Y^{(m)}(0)=Y(0) . \quad Y^{(m)}(t)$ evolves so that the environments $\{u(x, t)\}$ restrict its motion only on edges $\{x-1, x\}$ for $-m+1 \leq x \leq 0$. In other words, for walk $Y^{(m)}(t)$ we set $u(x, t) \equiv 1$ for $x \leq-m$ and $0 \leq t<\infty$. We couple the walks together so that $Y(t)=Y^{(m)}(t)$ until the first time one of the walks exits the interval $\{-m+1, \ldots, 0\}$.

Fixing $m$ for a moment, let $0=s_{0}<s_{1}<s_{2}<s_{3}<\ldots$ be a partition of the time axis so that $s_{j} \nearrow \infty$ and the environments $\{u(x, t):-m<$ $x \leq 0\}$ are constant on each interval $t \in\left[s_{i}, s_{i+1}\right)$. Then on each time 
interval $\left[s_{i}, s_{i+1}\right) Y^{(m)}(t)$ is a continuous time Markov chain with timehomogeneous jump rates

$$
c(x, x+1)= \begin{cases}p u\left(x+1, s_{i}\right), & -m \leq x \leq 0 \\ p, & x \leq-m-1\end{cases}
$$

and

$$
c(x, x-1)= \begin{cases}q u\left(x, s_{i}\right), & -m+1 \leq x \leq 0 \\ q, & x \leq-m .\end{cases}
$$

One can check that detailed balance $\pi(x) c(x, x+1)=\pi(x+1) c(x+1, x)$ holds for all $x \leq-1$. Thus $\pi$ is a reversible measure for walk $Y^{(m)}(t)$ on each time interval $\left[s_{i}, s_{i+1}\right)$, and we conclude that $Y^{(m)}(t)$ has distribution $\pi$ for all $0 \leq t<\infty$.

The coupling ensures that $Y^{(m)}(t) \rightarrow Y(t)$ almost surely as $m \rightarrow \infty$, and consequently also $Y(t)$ has distribution $\pi$ for all $0 \leq t<\infty$.

\subsection{Proof of the upper bound for second class particle moments}

Abbreviate

$$
\Psi(t)=\mathbf{E}^{\rho}\left|Q(t)-V^{\rho} t\right| .
$$

Lemma 5.3. Let $B \in(0, \infty)$. Then there exists a numerical constant $C \in(0, \infty)$ and another constant $c_{1}(B) \in(0, \infty)$ such that, for all densities $0<\rho<1, u \geq 1,0<\theta<1 / 2$, and $t \geq c_{1}(B) \theta^{-1}$,

$$
\begin{aligned}
& \mathbf{P}^{\rho}\left\{Q(t) \geq V^{\rho} t+u\right\} \\
& \quad \leq \begin{cases}C \theta^{2}\left(\frac{t^{2}}{u^{4}} \Psi(t)+\frac{t^{2}}{u^{3}}\right)+e^{-u^{2} / C t}, & B \theta^{2 / 3} t^{2 / 3} \leq u \leq 20 t / 3 \\
e^{-u / C}, & u \geq 20 t / 3 .\end{cases}
\end{aligned}
$$

Proof. First we get an easy case out of the way.

Case 1. $u \geq 5 \rho \theta t$.

This comes from an exponential Chebyshev argument. Let $Z_{t}$ be a nearest-neighbor random walk with rates $p=(1+\theta) / 2$ to the right and $q=(1-\theta) / 2$ to the left. For $\alpha \in(0,1]$, using

$$
\frac{e^{\alpha}+e^{-\alpha}}{2} \leq 1+\alpha^{2} \text { and } \quad \frac{e^{\alpha}-e^{-\alpha}}{2} \leq \alpha+\alpha^{2},
$$


we get

$$
\begin{aligned}
\mathbf{E}\left[e^{\alpha Z_{t}}\right] & =\exp \left(-t+t \frac{e^{\alpha}+e^{-\alpha}}{2}+\theta t \frac{e^{\alpha}-e^{-\alpha}}{2}\right) \\
& \leq \exp \left(2 \alpha^{2} t+\alpha \theta t\right) .
\end{aligned}
$$

We have the stochastic domination $Z_{t} \geq Q(t)$ because no matter what the environment next to $Q(t)$ is, $Q(t)$ has a weaker right drift than $Z_{t}$. Then, since $V^{\rho}=\theta(1-2 \rho)$ and $2 \rho \theta t \leq 2 u / 5$,

$$
\begin{aligned}
\mathbf{P}^{\rho}\left\{Q(t) \geq V^{\rho} t+u\right\} & \leq P\left\{Z_{t} \geq \theta t+\frac{3}{5} u\right\} \\
& \leq \exp \left(-\frac{3}{5} \alpha u+2 \alpha^{2} t\right) \\
& \leq \begin{cases}\exp \left(-\frac{9 u^{2}}{200 t}\right) & u \leq 20 t / 3 \\
\exp (-3 u / 10) & u>20 t / 3\end{cases}
\end{aligned}
$$

In the last inequality above choose $\alpha=1 \wedge \frac{3 u}{20 t}$. Note that $20 t / 3>5 \rho \theta t$.

It remains to consider this range of $u$ :

Case 2. $B \theta^{2 / 3} t^{2 / 3} \leq u \leq 5 \rho \theta t$.

By an adjustment of the constant $C$ we can assume that $u$ is a positive integer. Fix a density $0<\rho<1$ and an auxiliary density

$$
\lambda=\rho-\frac{u}{10 \theta t} .
$$

Start with the basic coupling of three exclusion processes $\omega \geq \omega^{-} \geq \eta$ with this initial set-up: 1 .

(a) Initially $\left\{\omega_{i}(0): i \neq 0\right\}$ are i.i.d. Bernoulli $(\rho)$ distributed and $\omega_{0}(0)=$

(b) Initially $\omega^{-}(0)=\omega(0)-\delta_{0}$.

(c) Initially variables $\left\{\eta_{i}(0): i \neq 0\right\}$ are i.i.d. Bernoulli $(\lambda)$ and $\eta_{0}(0)=0$. The coupling of the initial occupations is such that $\omega_{i}(0) \geq \eta_{i}(0)$ for all $i \neq 0$.

Recall that basic coupling meant that these processes obey common Poisson clocks.

Let $Q(t)$ be the position of the single second class particle between $\omega(t)$ and $\omega^{-}(t)$, initially at the origin. Let $\left\{X_{i}(t): i \in \mathbb{Z}\right\}$ be the positions of the $\omega-\eta$ second class particles, initially labeled so that

$$
\cdots<X_{-2}(0)<X_{-1}(0)<X_{0}(0)=0<X_{1}(0)<X_{2}(0)<\cdots
$$

These second class particles preserve their labels in the dynamics and stay ordered. Thus the $\omega(t)$ configuration consists of first class particles (the $\eta(t)$ process) and second class particles (the $X_{j}(t)$ 's). $\mathbf{P}$ denotes the probability measure under which all these coupled processes live. Note 
that the marginal distribution of $\left(\omega, \omega^{-}, Q\right)$ under $\mathbf{P}$ is exactly as it would be under $\mathbf{P}^{\rho}$.

For $x \in \mathbb{Z}, J_{x}^{\omega}(t)$ is the net current in the $\omega$-process between spacetime positions $(1 / 2,0)$ and $(x+1 / 2, t)$. Similarly $J_{x}^{\eta}(t)$ in the $\eta$-process, and $J_{x}^{\omega-\eta}(t)$ is the net current of second class particles. Current in the $\omega$-process is a sum of the first class particle current and the second class particle current:

$$
J_{x}^{\omega}(t)=J_{x}^{\eta}(t)+J_{x}^{\omega-\eta}(t) .
$$

$Q(t) \in\left\{X_{j}(t)\right\}$ for all time because the basic coupling preserves the ordering $\omega^{-}(t) \geq \eta(t)$. Define the label $m_{Q}(t)$ by $Q(t)=X_{m_{Q}(t)}(t)$ with initial value $m_{Q}(0)=0$

Lemma 5.4. For all $t \geq 0$ and $k \geq 0$,

$$
\mathbf{P}\left\{m_{Q}(t) \geq k\right\} \leq e^{-2 \theta k} .
$$

Proof of Lemma 5.4. In the basic coupling the label $m_{Q}(t)$ evolves as follows. When $X_{m_{Q}-1}$ is adjacent to $X_{m_{Q}}, m_{Q}$ jumps down by one at rate $p$. And when $X_{m_{Q}+1}$ is adjacent to $X_{m_{Q}}, m_{Q}$ jumps up by one at rate $q$. When $X_{m_{Q}}$ has no $X$-particle in either neighboring site, the label $m_{Q}$ cannot jump. Thus the situation is like that in Lemma 5.2 (with a reversal of lattice directions) with environment given by the adjacency of $X$-particles: $u(m, t)=\mathbf{1}\left\{X_{m}(t)=X_{m-1}(t)+1\right\}$. However, the basic coupling mixes together the evolution of the environment and the walk $m_{Q}$, so the environment is not specified in advance as required by Lemma 5.2.

We can get around this difficulty by imagining an alternative but distributionally equivalent construction for the joint process $\left(\eta, \omega^{-}, \omega\right)$. Let $(\eta, \omega)$ obey basic coupling with the given Poisson clocks $\left\{N^{x \rightarrow x \pm 1}\right\}$ attached to directed edges $(x, x \pm 1)$. Divide the $\omega-\eta$ particles further into class II consisting of the particles $\omega^{-}-\eta$ and class III that consists only of the single particle $\omega-\omega^{-}=\delta_{Q}$. Let class II have priority over class III. Introduce another independent set of Poisson clocks $\left\{\widetilde{N}^{x \rightarrow x \pm 1}\right\}$, also attached to directed edges $(x, x \pm 1)$ of the space $\mathbb{Z}$ where particles move. Let clocks $\left\{\widetilde{N}^{x \rightarrow x \pm 1}\right\}$ govern the exchanges between classes II and III. In other words, for each edge $\{x, x+1\}$ clocks $\widetilde{N}^{x \rightarrow x+1}$ and $\widetilde{N}^{x+1 \rightarrow x}$ are observed if sites $\{x, x+1\}$ are both occupied by $\omega-\eta$ particles. All other jumps are prompted by the original clocks.

The rates for individual jumps are the same in this alternative construction as in the earlier one where all processes were together in basic coupling. Thus the same distribution for the process $\left(\eta, \omega^{-}, \omega\right)$ is created.

To apply Lemma 5.2 perform the construction in two steps. First construct the process $(\eta, \omega)$ for all time. This determines the environment 
$u(m, t)=\mathbf{1}\left\{X_{m}(t)=X_{m-1}(t)+1\right\}$. Then run the dynamics of classes II and III in this environment. Now Lemma 5.2 gives the bound for $m_{Q}$.

Let $u$ be a positive integer and

$$
k=\left\lfloor\frac{u^{2}}{20 \theta t}\right\rfloor-3 .
$$

By assuming $t \geq C(B) \theta^{-1}$ we guarantee that

$$
u \geq 1 \text { and } \frac{u^{2}}{40 \theta t} \geq \frac{B^{2} \theta^{1 / 3} t^{1 / 3}}{40} \geq 4 .
$$

Then

$$
k \geq \frac{u^{2}}{40 \theta t} \geq 4
$$

We begin a series of inequalities.

$$
\begin{aligned}
& \mathbf{P}\left\{Q(t) \geq V^{\rho} t+u\right\} \\
& \leq \mathbf{P}\left\{m_{Q}(t) \geq k\right\}+\mathbf{P}\left\{J_{\left\lfloor V^{\rho} t\right\rfloor+u}^{\omega}(t)-J_{\left\lfloor V^{\rho} t\right\rfloor+u}^{\eta}(t)>-k\right\} .
\end{aligned}
$$

To explain the inequality above, if $Q(t) \geq V^{\rho} t+u$ and $m_{Q}(t)<k$ then $X_{k}(t)>\left\lfloor V^{\rho} t\right\rfloor+u$. This puts the bound

$$
J_{\left\lfloor V^{\rho} t\right\rfloor+u}^{\omega-\eta}(t)>-k
$$

on the second class particle current, because at most particles $X_{1}, \ldots, X_{k-1}$ could have made a negative contribution to this current.

Lemma 5.4 takes care of the first probability on line (5.31). We work on the second probability on line (5.31).

Here is a simple observation that will be used repeatedly. Process $\omega$ can be coupled with a stationary density- $\rho$ process $\omega^{(\rho)}$ so that the coupled pair $\left(\omega, \omega^{(\rho)}\right)$ has at most 1 discrepancy. In this coupling

$$
\left|J_{x}^{\omega}(t)-J_{x}^{\omega^{(\rho)}}(t)\right| \leq 1
$$

This way we can use computations for stationary processes at the expense of small errors.

Recall that $V^{\rho}=H^{\prime}(\rho)$. Let $c_{1}$ below be a constant that absorbs the errors from using means of stationary processes and from ignoring integer 
parts. It satisfies $\left|c_{1}\right| \leq 3$.

$$
\begin{aligned}
& \mathbf{E} J_{\left\lfloor V^{\rho} t\right\rfloor+u}^{\omega}(t)-\mathbf{E} J_{\left\lfloor V^{\rho} t\right\rfloor+u}^{\eta}(t) \\
& =t H(\rho)-\left(H^{\prime}(\rho) t+u\right) \rho-t H(\lambda)+\left(H^{\prime}(\rho) t+u\right) \lambda+c_{1} \\
& =-\frac{1}{2} t H^{\prime \prime}(\rho)(\rho-\lambda)^{2}-u(\rho-\lambda)+c_{1} \\
& =t \theta(\rho-\lambda)^{2}-u(\rho-\lambda)+c_{1} \\
& =t \theta(\rho-\lambda)^{2}-u(\rho-\lambda)+c_{1}+k-k \\
& \leq \frac{u^{2}}{100 t \theta}-\frac{u^{2}}{10 t \theta}+\frac{u^{2}}{20 t \theta}-k \\
& =-\frac{u^{2}}{25 t \theta}-k .
\end{aligned}
$$

The -3 in the definition (5.29) of $k$ absorbed $c_{1}$ above.

Let $\bar{X}=X-E X$ denote a centered random variable. Continuing with the second probability from line (5.31):

$$
\begin{aligned}
& \mathbf{P}\left\{J_{\left\lfloor V^{\rho} t\right\rfloor+u}^{\omega}(t)-J_{\left\lfloor V^{\rho} t\right\rfloor+u}^{\eta}(t)>-k\right\} \\
& \leq \mathbf{P}\left\{\bar{J}_{\left\lfloor V^{\rho} t\right\rfloor+u}^{\omega}(t)-\bar{J}_{\left\lfloor V^{\rho} t\right\rfloor+u}^{\eta}(t) \geq \frac{u^{2}}{25 t \theta}\right\} \\
& \leq \frac{C \theta^{2} t^{2}}{u^{4}} \operatorname{Var}\left\{J_{\left\lfloor V^{\rho} t\right\rfloor+u}^{\omega}(t)-J_{\left\lfloor V^{\rho} t\right\rfloor+u}^{\eta}(t)\right\} \\
& \leq \frac{C \theta^{2} t^{2}}{u^{4}}\left(\operatorname{Var}\left\{J_{\left\lfloor V^{\rho} t\right\rfloor+u}^{\omega}(t)\right\}+\operatorname{Var}\left\{J_{\left\lfloor V^{\rho} t\right\rfloor+u}^{\eta}(t)\right\}\right) .
\end{aligned}
$$

$C$ is a numerical constant that can change from line to line but is independent of all the parameters.

We develop bounds on the variances above, first for $J^{\omega}$. Pass to the stationary density- $\rho$ process via (5.32) and apply (5.11):

$$
\begin{aligned}
\operatorname{Var}\left\{J_{\left\lfloor V^{\rho} t\right\rfloor+u}^{\omega}(t)\right\} & \leq 2 \operatorname{Var}^{\rho}\left\{J_{\left\lfloor V^{\rho} t\right\rfloor+u}(t)\right\}+2 \\
& =2 \rho(1-\rho) \mathbf{E}\left|Q(t)-\left\lfloor V^{\rho} t\right\rfloor-u\right|+2 \\
& \leq \mathbf{E}\left|Q(t)-V^{\rho} t\right|+u+3 \\
& \leq \Psi(t)+4 u .
\end{aligned}
$$

Let $\operatorname{Var}^{\lambda}$ denote variance in the stationary density- $\lambda$ process and let $Q^{\eta}(t)$ denote the position of a second class particle added to a process $\eta$.

$$
\begin{aligned}
\operatorname{Var}\{ & \left.J_{\left\lfloor V^{\rho} t\right\rfloor+u}^{\eta}(t)\right\} \leq 2 \operatorname{Var}^{\lambda}\left\{J_{\left\lfloor V^{\rho} t\right\rfloor+u}(t)\right\}+2 \\
& \leq \mathbf{E}^{\lambda}\left|Q^{\eta}(t)-\left\lfloor V^{\rho} t\right\rfloor-u\right|+2 \\
& \leq \mathbf{E}^{\lambda}\left|Q^{\eta}(t)-V^{\rho} t\right|+4 u
\end{aligned}
$$


Introduce process $\left(\zeta^{-}(t), Q^{\zeta}(t), \eta(t), Q^{\eta}(t)\right)_{t \geq 0}$ coupled as in Theorem 5.6, where $\zeta$ starts with Bernoulli $(\rho)$ occupations away from the origin and initially $Q^{\zeta}(0)=Q^{\eta}(0)=0$. Below apply the triangle inequality and use inequality $Q^{\zeta}(t) \leq Q^{\eta}(t)$ from Theorem 5.6. Thus continuing from above:

$$
\begin{aligned}
& =\mathbf{E}\left|Q^{\eta}(t)-Q^{\zeta}(t)+Q^{\zeta}(t)-V^{\rho} t\right|+4 u \\
& \leq \mathbf{E}\left\{Q^{\eta}(t)-Q^{\zeta}(t)\right\}+\mathbf{E}\left|Q^{\zeta}(t)-V^{\rho} t\right|+4 u \\
& =V^{\lambda} t-V^{\rho} t+\Psi(t)+4 u \\
& =2 \theta t(\rho-\lambda)+\Psi(t)+4 u \\
& =\Psi(t)+5 u .
\end{aligned}
$$

Marginally the process $\left(\zeta, Q^{\zeta}\right)$ is the same as the process $(\omega, Q)$ in the coupling of this section, hence the appearance of $\Psi(t)$ above. Then we used (5.10) for the expectations of the second class particles and the choice (5.27) of $\lambda$.

Insert bounds (5.36) and (5.37) into (5.35) to get

$$
\mathbf{P}\left\{J_{\left\lfloor V^{\rho} t\right\rfloor+u}^{\omega}(t)-J_{\left\lfloor V^{\rho} t\right\rfloor+u}^{\eta}(t)>-k\right\} \leq C \theta^{2}\left(\frac{t^{2}}{u^{4}} \Psi(t)+\frac{t^{2}}{u^{3}}\right) .
$$

Insert (5.30) and (5.38) into line (5.31) to get

$$
\mathbf{P}\left\{Q(t) \leq V^{\rho} t-u\right\} \leq C \theta^{2}\left(\frac{t^{2}}{u^{4}} \Psi(t)+\frac{t^{2}}{u^{3}}\right)+e^{-u^{2} / 20 t}
$$

and we have verified (5.24) for Case 2 .

Combining (5.39) and (5.26) gives the conclusion of Lemma 5.3.

Next we extend the bound to both tails.

Lemma 5.5. Let $B \in(0, \infty)$. Then there exists a numerical constant $C \in(0, \infty)$ and another constant $c_{0}(B) \in(0, \infty)$ such that, for all densities $0<\rho<1$ and $t \geq c_{0}(B) \theta^{-1}$,

$$
\begin{aligned}
& \mathbf{P}^{\rho}\left\{\left|Q(t)-V^{\rho} t\right| \geq u\right\} \\
& \leq \begin{cases}C \theta^{2}\left(\frac{t^{2}}{u^{4}} \Psi(t)+\frac{t^{2}}{u^{3}}\right)+2 e^{-u^{2} / C t}, & B \theta^{2 / 3} t^{2 / 3} \leq u \leq 20 t / 3 \\
2 e^{-u / C}, & u \geq 20 t / 3 .\end{cases}
\end{aligned}
$$

Proof. The corresponding lower tail bound is obtained from (5.24) by a particle-hole interchange followed by a reflection of the lattice. For details we refer to Lemma 5.3 in [BS09a]. 
Proof of the upper bound of Theorem 5.4. We integrate (5.40) to get the bound (5.13) on the moments of the second class particle. First for $m=1$.

$$
\begin{gathered}
\Psi(t)=\int_{0}^{\infty} \mathbf{P}^{\rho}\left\{\left|Q(t)-V^{\rho} t\right| \geq u\right\} d u \\
\leq B \theta^{2 / 3} t^{2 / 3}+C \theta^{2} \int_{B \theta^{2 / 3} t^{2 / 3}}^{\infty}\left(\frac{t^{2}}{u^{4}} \Psi(t)+\frac{t^{2}}{u^{3}}\right) d u \\
\quad+2 \int_{B \theta^{2 / 3} t^{2 / 3}}^{\infty} e^{-u^{2} / C t} d u+2 \int_{20 t / 3}^{\infty} e^{-u / C} d u \\
\leq \frac{C}{3 B^{3}} \Psi(t)+\left(B+\frac{C}{2 B^{2}}\right) \theta^{2 / 3} t^{2 / 3} \\
\quad+\frac{C_{1}(B) t^{1 / 3}}{\theta^{2 / 3}} e^{-\theta^{4 / 3} t^{1 / 3} / C_{1}(B)}+2 C e^{-t / C} .
\end{gathered}
$$

$C_{1}(B)$ is a new constant that depends on $B$. Set $B=C^{1 / 3}$ to turn the above inequality into

$$
\Psi(t) \leq \frac{9 C^{1 / 3}}{4} \theta^{2 / 3} t^{2 / 3}+\frac{C_{1} t^{1 / 3}}{\theta^{2 / 3}} \exp \left(\frac{-\theta^{4 / 3} t^{1 / 3}}{C_{1}}\right)+2 C e^{-t / C} .
$$

The second term on the right above forces us to restrict $t$ further. We can fix a constant $c_{0}$ large enough so that, for a new constant $C$,

$$
\Psi(t) \leq C \theta^{2 / 3} t^{2 / 3} \quad \text { provided } t \geq c_{0} \theta^{-4} .
$$

Restrict to $t$ that satisfy this requirement and substitute this bound on $\Psi(t)$ into (5.40). Then upon using $u \geq B \theta^{2 / 3} t^{2 / 3}$ and redefining $C$ once more, we have for $B \theta^{2 / 3} t^{2 / 3} \leq u \leq 20 t / 3$ :

$$
\mathbf{P}^{\rho}\left\{\left|Q(t)-V^{\rho} t\right| \geq u\right\} \leq C \frac{\theta^{2} t^{2}}{u^{3}}+2 e^{-u^{2} / C t} .
$$

Now take $1<m<3$ and use (5.42) together with the second case of (5.40):

$$
\begin{aligned}
& \mathbf{E}^{\rho}\left|Q(t)-V^{\rho} t\right|^{m}=m \int_{0}^{\infty} \mathbf{P}^{\rho}\left\{\left|Q(t)-V^{\rho} t\right| \geq u\right\} u^{m-1} d u \\
& \leq B^{m} \theta^{2 m / 3} t^{2 m / 3}+C m \theta^{2} t^{2} \int_{B \theta^{2 / 3} t^{2 / 3}}^{\infty} u^{m-4} d u \\
& \quad+2 m \int_{B \theta^{2 / 3} t^{2 / 3}}^{\infty} e^{-u^{2} / C t} u^{m-1} d u+2 m \int_{20 t / 3}^{\infty} e^{-u / C} u^{m-1} d u .
\end{aligned}
$$

Performing and approximating the integrals gives

$$
\mathbf{E}^{\rho}\left|Q(t)-V^{\rho} t\right|^{m} \leq \frac{C}{3-m} \theta^{2 m / 3} t^{2 m / 3}
$$

provided $t \geq c_{0} \theta^{-4}$ for a large enough constant $c_{0}$. 


\subsection{Proof of the lower bound for second class particle moments}

By Jensen's inequality it suffices to prove the lower bound for $m=1$. Let $C_{U B}$ denote the constant in the upper bound statement that we just proved. We can also assume $c_{0} \geq 1$. Fix a constant $b>0$ and set

$$
a_{1}=2 C_{U B}+1 \quad \text { and } \quad a_{2}=8+\sqrt{32 b}+8 \sqrt{C_{U B}} .
$$

Increase $b$ if necessary so that

$$
b^{2}-2 a_{2} \geq 1
$$

Fix a density $\rho \in(0,1)$ and define an auxiliary density $\lambda=\rho-b t^{-1 / 3} \theta^{-1 / 3}$. Define positive integers

$$
u=\left\lfloor a_{1} t^{2 / 3} \theta^{2 / 3}\right\rfloor \quad \text { and } \quad n=\left\lfloor V^{\lambda} t\right\rfloor-\left\lfloor V^{\rho} t\right\rfloor+u .
$$

By taking $c_{0}$ large enough in the statement of Theorem 5.4 we can ensure that $\lambda \in(\rho / 2, \rho)$ and $u \in \mathbb{N}$.

Construct a basic coupling of three processes $\eta \leq \eta^{+} \leq \zeta$ with the following initial state:

(a) Initially $\eta$ has i.i.d. Bernoulli $(\lambda)$ occupations $\left\{\eta_{i}(0): i \neq-n\right\}$ and $\eta_{-n}(0)=0$.

(b) Initially $\eta^{+}(0)=\eta(0)+\delta_{-n} \cdot Q^{(-n)}(t)$ is the location of the unique discrepancy between $\eta(t)$ and $\eta^{+}(t)$.

(c) Initially $\zeta$ has independent occupation variables, coupled with $\eta(0)$ as follows:

(c.1) $\zeta_{i}(0)=\eta_{i}(0)$ for $-n<i \leq 0$.

(c.2) $\zeta_{-n}(0)=1$.

(c.3) For $i<-n$ and $i>0$ variables $\zeta_{i}(0)$ are i.i.d. Bernoulli $(\rho)$ and $\zeta_{i}(0) \geq \eta_{i}(0)$.

Thus the initial density of $\zeta$ is piecewise constant: on the segment $\{-n+$ $1, \ldots, 0\} \zeta(0)$ is i.i.d. with density $\lambda$, at site $-n \zeta(0)$ has density 1 , and elsewhere on $\mathbb{Z} \zeta(0)$ is i.i.d. with density $\rho$. The reason for the gap in the $\zeta-\eta$ second class particles across $(-n, 0]$ is to get an upper bound on the second-class particle current that is not too large for subsequent arguments ((5.48) below).

Label the $\zeta-\eta$ second class particles as $\left\{Y_{m}(t): m \in \mathbb{Z}\right\}$ so that initially

$$
\cdots<Y_{-1}(0)<Y_{0}(0)=-n=Q^{(-n)}(0)<0<Y_{1}(0)<Y_{2}(0)<\cdots
$$

Let again $m_{Q}(t)$ be the label such that $Q^{(-n)}(t)=Y_{m_{Q}(t)}(t)$. Initially $m_{Q}(0)=0$. The inclusion $Q^{(-n)}(t) \in\left\{Y_{m}(t)\right\}$ persists for all time because 
the basic coupling preserves the ordering $\zeta(t) \geq \eta^{+}(t)$. Through the basic coupling $m_{Q}$ jumps to the left with rate $q$ and to the right with rate $p$, but only when there is a $Y$-particle adjacent to $Y_{m_{Q}}$. As in the proof of Lemma 5.4 we can apply Lemma 5.2 to prove this statement:

$$
\mathbf{P}\left\{m_{Q}(t) \leq-k\right\} \leq e^{-2 \theta k} .
$$

By the upper bound already proved and by the choice of $a_{1}$,

$$
\begin{aligned}
& \mathbf{P}\left\{Q^{(-n)}(t) \geq\left\lfloor V^{\rho} t\right\rfloor\right\}=\mathbf{P}\left\{Q^{(-n)}(t) \geq-n+\left\lfloor V^{\lambda} t\right\rfloor+u\right\} \\
& \quad \leq u^{-1} \mathbf{E}\left|Q^{(-n)}(t)-n-\left\lfloor V^{\lambda} t\right\rfloor\right| \leq \frac{C_{U B} t^{2 / 3} \theta^{2 / 3}}{\left\lfloor a_{1} t^{2 / 3} \theta^{2 / 3}\right\rfloor} \\
& \quad \leq \frac{1}{2}
\end{aligned}
$$

For the complementary event we get a lower bound:

$$
\begin{aligned}
\frac{1}{2} & \leq \mathbf{P}\left\{Q^{(-n)}(t) \leq\left\lfloor V^{\rho} t\right\rfloor\right\} \\
& \leq \mathbf{P}\{m(t) \leq-k\}+\mathbf{P}\left\{J_{\left\lfloor V^{\rho} t\right\rfloor}^{\zeta}(t)-J_{\left\lfloor V^{\rho} t\right\rfloor}^{\eta}(t) \leq k\right\} .
\end{aligned}
$$

The reasoning behind the second inequality above is this. If $Q^{(-n)}(t) \leq$ $\left\lfloor V^{\rho} t\right\rfloor$ and $m_{Q}(t)>-k$ then $Y_{-k}(t) \leq\left\lfloor V^{\rho} t\right\rfloor$. This implies a bound on the second class particle current:

$$
J_{\left\lfloor V^{\rho} t\right\rfloor}^{\zeta}(t)-J_{\left\lfloor V^{\rho} t\right\rfloor}^{\eta}(t)=J_{\left\lfloor V^{\rho} t\right\rfloor}^{\zeta-\eta}(t) \leq k .
$$

Put $k=\left\lfloor a_{2} t^{1 / 3} \theta^{1 / 3}\right\rfloor-2$. Then by $t \geq \theta^{-4}$ and the definition of $a_{2}$,

$$
\mathbf{P}\left\{m_{Q}(t) \leq-k\right\} \leq e^{-2}<1 / 4
$$

Combine (5.47) and (5.49) and split the probability:

$$
\begin{aligned}
\frac{1}{4} \leq \mathbf{P}\left\{J_{\left\lfloor V^{\rho} t\right\rfloor}^{\zeta}(t)-J_{\left\lfloor V^{\rho} t\right\rfloor}^{\eta}(t) \leq a_{2} t^{1 / 3} \theta^{1 / 3}-2\right\} \\
\leq \mathbf{P}\left\{J_{\left\lfloor V^{\rho} t\right\rfloor}^{\zeta}(t) \leq 2 a_{2} t^{1 / 3} \theta^{1 / 3}+t \theta\left(2 \rho \lambda-\lambda^{2}\right)\right\} \\
\quad+\mathbf{P}\left\{J_{\left\lfloor V^{\rho} t\right\rfloor}^{\eta}(t) \geq a_{2} t^{1 / 3} \theta^{1 / 3}+t \theta\left(2 \rho \lambda-\lambda^{2}\right)+2\right\} .
\end{aligned}
$$

Consider next line (5.50). The $\eta$-process can be coupled with a stationary $P^{\lambda}$-process with at most one discrepancy. The mean current in the stationary process is

$$
\begin{aligned}
E^{\lambda}\left[J_{\left\lfloor V^{\rho} t\right\rfloor}(t)\right] & =t H(\lambda)-\lambda\left\lfloor V^{\rho} t\right\rfloor \\
& \leq t H(\lambda)-\lambda V^{\rho} t+1=t \theta\left(2 \rho \lambda-\lambda^{2}\right)+1
\end{aligned}
$$


Hence

$$
\begin{aligned}
& \text { line }(5.50) \leq P^{\lambda}\left\{J_{\left\lfloor V^{\rho} t\right\rfloor}(t) \geq a_{2} t^{1 / 3} \theta^{1 / 3}+t \theta\left(2 \rho \lambda-\lambda^{2}\right)+1\right\} \\
& \leq P^{\lambda}\left\{\bar{J}_{\left\lfloor V^{\rho} t\right\rfloor}(t) \geq a_{2} t^{1 / 3} \theta^{1 / 3}\right\} \leq a_{2}^{-2} t^{-2 / 3} \theta^{-2 / 3} \operatorname{Var}^{\lambda}\left[J_{\left\lfloor V^{\rho} t\right\rfloor}(t)\right] \\
& \leq \frac{\mathbf{E}^{\lambda}\left|Q(t)-\left\lfloor V^{\rho} t\right\rfloor\right|}{a_{2}^{2} t^{2 / 3} \theta^{2 / 3}} \leq \frac{\mathbf{E}^{\lambda}\left|Q(t)-V^{\lambda} t\right|}{a_{2}^{2} t^{2 / 3} \theta^{2 / 3}}+\frac{2 b}{a_{2}^{2}}+\frac{1}{a_{2}^{2} t^{2 / 3} \theta^{2 / 3}} \\
& \leq C_{U B} a_{2}^{-2}+\frac{1}{16}+\frac{1}{64} \leq \frac{1}{8}
\end{aligned}
$$

After Chebyshev above we applied the basic identity (5.11) for which we introduced a second class particle $Q(t)$ in a density- $\lambda$ system under the measure $\mathbf{P}^{\lambda}$. Then we replaced $\left\lfloor V^{\rho} t\right\rfloor$ with $V^{\lambda} t$ and applied the upper bound and properties of $a_{2}$.

Put this last bound back into line (5.50) to be left with

$$
\frac{1}{8} \leq \mathbf{P}\left\{J_{\left[V^{\rho} t\right]}^{\zeta}(t) \leq 2 a_{2} t^{1 / 3} \theta^{1 / 3}+t \theta\left(2 \rho \lambda-\lambda^{2}\right)\right\} .
$$

Next we replace the $\zeta$-process with a stationary density- $\rho$ process by inserting the Radon-Nikodym factor. Let $\gamma$ denote the distribution of the initial $\zeta(0)$ configuration described by $(\mathrm{c} 1)-(\mathrm{c} 3)$ in the beginning of this section. As before $\nu^{\rho}$ is the density- $\rho$ i.i.d. Bernoulli measure. The Radon-Nikodym derivative is

$f(\omega)=\frac{d \gamma}{d \nu^{\rho}}(\omega)=\frac{1}{\rho} \mathbf{1}\left\{\omega_{-n}=1\right\} \cdot \prod_{i=-n+1}^{0}\left(\frac{\lambda}{\rho} \mathbf{1}\left\{\omega_{i}=1\right\}+\frac{1-\lambda}{1-\rho} \mathbf{1}\left\{\omega_{i}=0\right\}\right)$.

Bound its second moment:

$$
E^{\rho}\left(f^{2}\right)=\frac{1}{\rho}\left(1+\frac{(\rho-\lambda)^{2}}{\rho(1-\rho)}\right)^{n} \leq \rho^{-1} e^{n(\rho-\lambda)^{2} / \rho(1-\rho)} \leq c_{2}(\rho)
$$

where condition $t \geq c_{0} \theta^{-4}$ implies a bound $c_{2}(\rho)<\infty$ independent of $t$ and $\theta$.

Let $\mathcal{A}$ denote the exclusion process event

$$
\mathcal{A}=\left\{J_{\left\lfloor V^{\rho} t\right\rfloor}(t) \leq 2 a_{2} t^{1 / 3} \theta^{1 / 3}+t \theta\left(2 \rho \lambda-\lambda^{2}\right)\right\} .
$$

Then from (5.52)

$$
\begin{aligned}
\frac{1}{8} & \leq \mathbf{P}\{\zeta \in \mathcal{A}\}=\int P^{\omega}(\mathcal{A}) \gamma(d \omega)=\int P^{\omega}(\mathcal{A}) f(\omega) \nu^{\rho}(d \omega) \\
& \leq\left(P^{\rho}(\mathcal{A})\right)^{1 / 2}\left(E^{\rho}\left(f^{2}\right)\right)^{1 / 2} \leq c_{2}(\rho)^{1 / 2}\left(P^{\rho}(\mathcal{A})\right)^{1 / 2} .
\end{aligned}
$$

Note the stationary mean

$$
E^{\rho}\left[J_{\left\lfloor V^{\rho} t\right\rfloor}(t)\right]=t H(\rho)-\rho\left\lfloor V^{\rho} t\right\rfloor=t \theta \rho^{2}+\rho V^{\rho} t-\rho\left\lfloor V^{\rho} t\right\rfloor \geq t \theta \rho^{2} .
$$


Continue from line (5.54), recalling (5.43):

$$
\begin{aligned}
\left(64 c_{2}(\rho)\right)^{-1} & \leq P^{\rho}(\mathcal{A})=P^{\rho}\left\{J_{\left\lfloor V^{\rho} t\right\rfloor}(t) \leq 2 a_{2} t^{1 / 3} \theta^{1 / 3}+t \theta\left(2 \rho \lambda-\lambda^{2}\right)\right\} \\
& \leq P^{\rho}\left\{\bar{J}_{\left\lfloor V^{\rho} t\right\rfloor}(t) \leq 2 a_{2} t^{1 / 3} \theta^{1 / 3}-t \theta(\rho-\lambda)^{2}\right\} \\
& =P^{\rho}\left\{\bar{J}_{\left\lfloor V^{\rho} t\right\rfloor}(t) \leq-\left(b^{2}-2 a_{2}\right) t^{1 / 3} \theta^{1 / 3}\right\} \\
& \leq P^{\rho}\left\{\bar{J}_{\left\lfloor V^{\rho} t\right\rfloor}(t) \leq-t^{1 / 3} \theta^{1 / 3}\right\} \\
& \leq t^{-2 / 3} \theta^{-2 / 3} \operatorname{Var}^{\rho}\left[J_{\left\lfloor V^{\rho} t\right\rfloor}(t)\right] \leq t^{-2 / 3} \theta^{-2 / 3} \mathbf{E}^{\rho}\left|Q(t)-V^{\rho} t\right| .
\end{aligned}
$$

This completes the proof of the lower bound. We finish with some observations about the need for the two key assumptions, asymmetry and $H^{\prime \prime}(\rho) \neq 0$.

For symmetric SEP $\theta=0$ and consequently the Chebyshev step above cannot be taken.

To observe where $H^{\prime \prime}(\rho)<0$ came in we need to backtrack a little. At stage (5.52) we have the inequality (ignoring now small errors due to integer parts etc.)

$$
\frac{1}{8} \leq \mathbf{P}\left\{J_{\left\lfloor V^{\rho} t\right\rfloor}^{\zeta}(t) \leq 2 a_{2} t^{1 / 3} \theta^{1 / 3}+E^{\lambda}\left(J_{\left\lfloor V^{\rho} t\right\rfloor}(t)\right)\right\} .
$$

The Radon-Nikodym and Schwarz trick turned this into an inequality for a stationary process:

$$
\begin{gathered}
0<c \leq P^{\rho}\left\{J_{\left\lfloor V^{\rho} t\right\rfloor}(t) \leq 2 a_{2} t^{1 / 3} \theta^{1 / 3}+E^{\lambda}\left(J_{\left\lfloor V^{\rho} t\right\rfloor}(t)\right)\right\} \\
=P^{\rho}\left\{\bar{J}_{\left\lfloor V^{\rho} t\right\rfloor}(t) \leq 2 a_{2} t^{1 / 3} \theta^{1 / 3}+E^{\lambda}\left(J_{\left\lfloor V^{\rho} t\right\rfloor}(t)\right)-E^{\rho}\left(J_{\left\lfloor V^{\rho} t\right\rfloor}(t)\right)\right\} .
\end{gathered}
$$

Compute the means on the right-hand side inside the probability, remembering that $V^{\rho}=H^{\prime}(\rho)$ and Taylor expanding $H(\lambda)$ :

$$
\begin{aligned}
E^{\lambda}\left(J_{\left\lfloor V^{\rho} t\right\rfloor}(t)\right)-E^{\rho}\left(J_{\left\lfloor V^{\rho} t\right\rfloor}(t)\right) & =t\left[H(\lambda)-\lambda H^{\prime}(\rho)-H(\rho)+\rho H^{\prime}(\rho)\right] \\
& =t\left[\frac{1}{2} H^{\prime \prime}(\rho)(\lambda-\rho)^{2}+O\left(\theta|\lambda-\rho|^{3}\right)\right] \\
& =-a_{3} b^{2} t^{1 / 3} \theta^{1 / 3}+O(1)
\end{aligned}
$$

with $\frac{1}{2} H^{\prime \prime}(\rho)=-a_{3} \theta<0$ in the last step. Thus the constants can be adjusted so that the probability in (5.55) is a deviation. Chebyshev can be applied to conclude that the current variance is of order $t^{2 / 3} \theta^{2 / 3}$. But if $H^{\prime \prime}(\rho)=0$ there is no deviation to take advantage of.

\section{Further comments and references}

The proofs of this chapter are based on [BS09a]. This article gives simpler proofs for the results in [BS07] and [BS09b]. Precursors of these variance bounds were first proved for last-passage models that correspond to 
totally asymmetric versions of particle systems: in [CG06] for the Hammersley process and in [BCS06] for the corner growth model associated with TASEP.

The Tracy-Widom type limit distribution for TASEP current was first proved for the step initial condition in [Joh00], then for the stationary case (Theorem 5.5) in [FS06]. The larger picture of TASEP fluctuations from various initial conditions is presented in [BAC09].

Another line of work has produced comparison theorems that allow one to conclude that Laplace transforms of $t^{-1} \operatorname{Var}^{\rho}[Q(t)]$ for different asymmetric exclusion processes are within constant multiples of each other. In this sense, for the order of this Laplace transform there is universality for all finite range asymmetric exclusion processes. These results come from resolvent techniques [Set03, QV07, QV08].

The central limit theorem for the current in directions other than the characteristic $V^{\rho}$ was proved first by Ferrari and Fontes [FF94]. This was generalized to other particle systems such as certain zero-range and bricklayer processes by Balázs [Bal03].

Consider symmetric simple exclusion, namely the case $p=q=1 / 2$. Then $V^{\rho}=0$. Equation (5.11) together with the observation that the second class particle is a simple symmetric random walk tell us that $\operatorname{Var}^{\rho}\left[J_{0}(t)\right]$ is of order $t^{1 / 2}$, exactly as for independent particles in Section 2. And indeed the current process does converge to fractional Brownian motion (see [PS08] and its references). 


\section{Chapter 6}

\section{Zero range process}

\subsection{Model and results}

From the perspective of universality it would be highly desirable to extend the results of Section 5 beyond exclusion processes. Throughout the 40year history of the subject of interacting particle systems, the zero range process has been a much-studied relative of the exclusion process. In this section we indicate how the bounds for second class particles and current variance are proved for a class of totally asymmetric zero range processes (TAZRP) with concave jump rate functions.

Definition and graphical construction. In contrast with the exclusion process, the zero range process does not restrict the number of particles allowed at a site. The state of the process at time $t$ is $\eta(t)=$ $\left(\eta_{i}(t)\right)_{i \in \mathbb{Z}} \in \mathbb{Z}_{+}^{\mathbb{Z}}$ where $\eta_{i}(t) \in \mathbb{Z}_{+}$denotes the number of particles present at site $i$ at time $t$. We consider the case where particles take only nearestneighbor jumps to the right.

Each zero range process is characterized by a jump rate function $g$ : $\mathbb{Z}_{+} \rightarrow \mathbb{R}_{+}$. It automatically has the value $g(0)=0$. The meaning of $g$ for the process is that when the current state is $\eta, g\left(\eta_{i}\right)$ is the rate at which one particle is moved from site $i$ to $i+1$. You can interpret this as saying that each of the particles at site $i$ jumps independently with rate $\eta_{i}^{-1} g\left(\eta_{i}\right)$ or that some particular one moves next (say, the bottom one is moved to the top of the next pile) at rate $g\left(\eta_{i}\right)$. It is immaterial for we do not label our particles. (Except again we will label certain second class particles as we did for ASEP, but we will come to that later.) These jump events take place independently at all sites, exactly as for ASEP.

We shall assume that

$$
g \text { is nondecreasing and } 0<g(k) \leq 1 \text { for } k>0 \text {. }
$$


With a bounded $g$ we can perform the following concrete construction of the process $\eta(t)$ in terms of independent rate 1 Poisson processes $\left\{N_{i}\right\}$ and i.i.d. Uniform $(0,1)$ variables $\left\{U_{i, k}\right\}$. Attach clock $N_{i}$ to site $i$, and give each jump time of $N_{i}$ its own $U_{i, k}$. Now if $t$ is a jump time for $N_{i}$ with its uniform $U_{i, k}$, then move one particle from $i$ to $i+1$ if $U_{i, k}<g\left(\eta_{i}(t-)\right)$, otherwise not. Repeat this step at all sites and all jump times. The result is the desired one: independently at each site $i$, a jump from $i$ to $i+1$ occurs in the next small time interval $(t, t+d t)$ with probability $g\left(\eta_{i}(t)\right) d t+O\left(d t^{2}\right)$

The generator of this TAZRP is

$$
L \varphi(\eta)=\sum_{i \in \mathbb{Z}} g\left(\eta_{i}\right)\left[\varphi\left(\eta^{i, i+1}\right)-\varphi(\eta)\right]
$$

that acts on bounded cylinder functions $\varphi$ on $\mathbb{Z}_{+}^{\mathbb{Z}}$ and $\eta^{i, i+1}=\eta-\delta_{i}+$ $\delta_{i+1}$. We will not use the generator in the text. It can be used to check the invariance of certain distributions on the state space, as for ASEP in Remark 5.2.

Invariant distributions. Part of the reason for the popularity of ZRP is that, just like ASEP, it has i.i.d. invariant distributions. We denote these by $\left\{\nu^{\rho}\right\}_{0 \leq \rho<\infty}$ indexed by density $\rho=E^{\rho}\left(\eta_{i}\right)$.

Here is the definition of these measures. Let $\theta$ denote a real parameter, and on $\mathbb{Z}_{+}$define a probability distribution

$$
\lambda^{\theta}(k)=\frac{1}{Z_{\theta}} \cdot \frac{e^{\theta k}}{g(k) !},
$$

defined for $\theta$ such that

$$
Z_{\theta}=\sum_{k} \frac{e^{\theta k}}{g(k) !}<\infty .
$$

Here $g(0) !=1$ and $g(k) !=g(1) \cdots g(k)$ for $k>0$. Define the mean density function $\rho(\theta)=\sum_{k} k \lambda^{\theta}(k)$. It is smooth and strictly increasing on the open interval where $Z_{\theta}<\infty$. Let its inverse function be $\theta(\rho)$ and then reparametrize the distributions in terms of density:

$$
\nu_{0}^{\rho}(k)=\lambda^{\theta(\rho)}(k)=\frac{1}{Z_{\theta(\rho)}} \cdot \frac{e^{\theta(\rho) k}}{g(k) !} .
$$

Finally, the actual invariant measures for ZRP are the product measures on the state space $\mathbb{Z}_{+}^{\mathbb{Z}}$ :

$$
\nu^{\rho}(d \eta)=\bigotimes_{i \in \mathbb{Z}} \nu_{0}^{\rho}\left(d \eta_{i}\right)
$$


We write $P^{\rho}$ for probabilities and $E^{\rho}$ for expectations for the stationary process whose marginal $\eta(t)$ has distribution $\nu^{\rho}$.

Basic coupling and second class particles. Basic coupling works exactly as it did for exclusion processes: two (or more) zero range processes obey a common set of Poisson clocks $\left\{N_{i}\right\}$ and uniform variables $\left\{U_{i, k}\right\}$.

We write a boldface $\mathbf{P}$ for the probability measure when more than one process are coupled together. In particular, $\mathbf{P}^{\rho}$ represents the situation where the initial occupation variables $\eta_{i}(0)=\eta_{i}^{+}(0)$ are i.i.d. mean- $\rho$ Bernoulli for $i \neq 0$, and the second class particle $Q$ starts at $Q(0)=0$.

More generally, if two processes $\eta$ and $\omega$ are in basic coupling and $\omega(0) \geq$ $\eta(0)$ (by which we mean coordinatewise ordering $\omega_{i}(0) \geq \eta_{i}(0)$ for all $i$ ) then the ordering $\omega(t) \geq \eta(t)$ holds for all $0 \leq t<\infty$. The effect of the basic coupling is to give priority to the $\eta$ particles over the $\omega-\eta$ particles. Consequently we can think of the $\omega$-process as consisting of first class particles (the $\eta$ particles) and second class particles (the $\omega-\eta$ particles).

Current. The current is defined as for ASEP: for $x \in \mathbb{Z}$ and $t>0, J_{x}(t)$ is the net left-to-right particle current across the straight-line space-time path from $(1 / 2,0)$ to $(x+1 / 2, t)$.

The flux function is again

$$
H(\rho)=E^{\rho}[\text { rate of particle flow across a fixed edge }]=E^{\rho}\left[g\left(\eta_{i}\right)\right] .
$$

Expectations of currents can be computed as for ASEP:

$$
E^{\rho}\left[J_{x}(t)\right]=t H(\rho)-x \rho, \quad x \in \mathbb{Z}, t \geq 0 .
$$

The characteristic speed at density $\rho$ is defined the same way as before:

$$
V^{\rho}=H^{\prime}(\rho)
$$

As for ASEP, the first task is to establish the identities that connect current variance and the second class particle. These identities for ZRP develop the same way as for ASEP, except that a new initial distribution for the coupled process appears. Define a probability distribution $\hat{\nu}_{0}^{\rho}$ on $\mathbb{Z}_{+}$by

$$
\hat{\nu}_{0}^{\rho}(k)=\frac{1}{\operatorname{Var}^{\rho}\left(\eta_{0}\right)} \sum_{m=k+1}^{\infty}(m-\rho) \nu_{0}^{\rho}(m), \quad k \in \mathbb{Z}_{+} .
$$

Define a product distribution $\hat{\nu}^{\rho}$ on the state space $\mathbb{Z}_{+}^{\mathbb{Z}}$ that obeys the marginals $\nu_{0}^{\rho}$ of the stationary distribution at all sites except at the origin where the distribution is $\hat{\nu}_{0}^{\rho}$ :

$$
\hat{\nu}^{\rho}(d \eta)=\left(\bigotimes_{i \neq 0} \nu_{0}^{\rho}\left(d \eta_{i}\right)\right) \otimes \hat{\nu}_{0}^{\rho}\left(d \eta_{0}\right) .
$$


Let $\widehat{\mathbf{P}}^{\rho}$ be the probability distribution of a pair $\left(\eta, \eta^{+}\right)$that satisfies $\eta^{+}(t)=\eta(t)+\delta_{Q(t)}$ (so there is one discrepancy), obeys basic coupling, and whose initial distribution is such that $\eta(0) \sim \hat{\nu}^{\rho}$ and $\eta^{+}(0)=\eta(0)+\delta_{0}$ (in other words, $Q(0)=0$ ).

Theorem 6.1. For any density $0<\rho<\infty, z \in \mathbb{Z}$ and $t>0$ we have these formulas.

$$
\begin{gathered}
\operatorname{Var}^{\rho}\left[J_{z}(t)\right]=\sum_{j \in \mathbb{Z}}|j-z| \operatorname{Cov}^{\rho}\left[\eta_{j}(t), \eta_{0}(0)\right], \\
\operatorname{Cov}^{\rho}\left[\eta_{j}(t), \eta_{0}(0)\right]=\operatorname{Var}^{\rho}\left(\eta_{0}\right) \widehat{\mathbf{P}}^{\rho}\{Q(t)=j\},
\end{gathered}
$$

and

$$
\widehat{\mathbf{E}}^{\rho}[Q(t)]=V^{\rho} t
$$

Equation (6.5) is proved the same way as for ASEP. Equation (6.6) and the definition of $\hat{\nu}_{0}^{\rho}$ come from a short calculation which we show below in Section 6.2. We omit the proof of (6.7). Formulas (6.5) and (6.6) combine to give the key equation that links current variance with the second class particle:

$$
\operatorname{Var}^{\rho}\left[J_{z}(t)\right]=\operatorname{Var}^{\rho}\left(\eta_{0}\right) \widehat{\mathbf{E}}^{\rho}|Q(t)-z| .
$$

Next we state the main result which again consists of upper and lower moment bounds for a second class particle, this time under the measure $\widehat{\mathbf{P}}^{\rho}$. We need a significant restriction on the concavity of the jump rate $g$ :

$$
\exists 0<r<1 \text { such that } g(k+1)-g(k) \leq r(g(k)-g(k-1)) .
$$

A class of examples satisfying this hypotheses is given by $g(k)=1-$ $\exp \left(-a k^{b}\right)$ with $a>0, b \geq 1 . g(k)$ can also be constant from some $k_{0}$ onwards.

Theorem 6.2. Fix a density $0<\rho<\infty$ and consider a pair of coupled ZRP's under the measure $\widehat{\mathbf{P}}^{\rho}$. Assume the jump rate function $g$ satisfies (6.1) and (6.9). Then for $1 \leq m<3$, large enough $t \in \mathbb{R}_{+}$and a constant C,

$$
\frac{1}{C} t^{2 m / 3} \leq \widehat{\mathbf{E}}^{\rho}\left[\left|Q(t)-V^{\rho} t\right|^{m}\right] \leq \frac{C}{3-m} t^{2 m / 3} .
$$

The constants $C$ and how large $t$ needs to be may depend on the density $\rho$. Combining (6.5) and (6.10) gives the bounds for the variance of the current seen by an observer traveling at the characteristic speed $V^{\rho}$ : for large enough $t$,

$$
C^{-1} t^{2 / 3} \leq \operatorname{Var}^{\rho}\left[J_{\left\lfloor V^{\rho} t\right\rfloor}(t)\right] \leq C t^{2 / 3}
$$


For the remainder of this chapter we discuss parts of the proof. Once we have the fundamental identities that tie together moments of the second class particle and the variance of the current, the proofs for the upper and lower bounds given for ASEP in Sections 5.5 and 5.6 can be adjusted to work for TAZRP. We will not repeat those derivations. Instead, we focus on the key ingredients that made the argument work for ASEP, and discuss how to provide these ingredients for TAZRP. There are two key points that we need in order to repeat the argument for TAZRP:

1. We need a construction that includes a given second class particle as a labeled member of a density of second class particles, and then we need a tail bound for the label as the one given for ASEP in Lemma 5.4 and (5.45).

2. We need a coupling that keeps the second class particle of a system with higher density behind the second class particle of a system with lower density. For ASEP this was Theorem 5.6, which was used to obtain (5.37) for the ASEP upper bound.

We turn to these points in Section 6.3 below, after developing the variance formula.

\subsection{Variance identity}

Define $F(-1)=0$ and

$$
F(k)=\sum_{m=k+1}^{\infty}(m-\rho) \frac{\nu_{0}^{\rho}(m)}{\nu_{0}^{\rho}(k)}=\frac{\operatorname{Var}^{\rho}\left(\eta_{0}\right)}{\nu_{0}^{\rho}(k)} \hat{\nu}_{0}^{\rho}(k), \quad k \geq 0 .
$$

Interpret below $\nu_{0}^{\rho}(-1)$ as 0 .

$$
\begin{aligned}
& \operatorname{Cov}^{\rho}\left[\eta_{i}(t), \eta_{0}(0)\right]=E^{\rho}\left[\eta_{i}(t)\left(\eta_{0}(0)-\rho\right)\right] \\
= & \sum_{k \geq 0} E\left[\eta_{i}(t) \mid \eta_{0}(0)=k\right](k-\rho) \nu_{0}^{\rho}(k) \\
= & \sum_{k \geq 0} E\left[\eta_{i}(t) \mid \eta_{0}(0)=k\right]\left(F(k-1) \nu_{0}^{\rho}(k-1)-F(k) \nu_{0}^{\rho}(k)\right) \\
= & \sum_{k \geq 0} E\left[\eta_{i}(t) \mid \eta_{0}(0)=k+1\right] F(k) \nu_{0}^{\rho}(k) \\
& -\sum_{k \geq 0} E\left[\eta_{i}(t) \mid \eta_{0}(0)=k\right] F(k) \nu_{0}^{\rho}(k) .
\end{aligned}
$$


Construct a coupling of $\eta^{+}(t)=\eta(t)+\delta_{Q(t)}$ with the discrepancy initially at the origin $Q(0)=0$, and so that $\eta(0)$ has $\nu^{\rho}$-distribution. Then, due to the product form of the initial distribution,

$$
\mathbf{E}\left[\eta_{i}(t) \mid \eta_{0}(0)=k+1\right]=\mathbf{E}\left[\eta_{i}^{+}(t) \mid \eta_{0}^{+}(0)=k+1\right]=\mathbf{E}\left[\eta_{i}^{+}(t) \mid \eta_{0}(0)=k\right] .
$$

Then continuing from above,

$$
\begin{aligned}
\operatorname{Cov}^{\rho}\left[\eta_{i}(t), \eta_{0}(0)\right]= & \sum_{k \geq 0} \mathbf{E}\left[\eta_{i}^{+}(t) \mid \eta_{0}(0)=k\right] F(k) \nu_{0}^{\rho}(k) \\
& \quad-\sum_{k \geq 0} \mathbf{E}\left[\eta_{i}(t) \mid \eta_{0}(0)=k\right] F(k) \nu_{0}^{\rho}(k) \\
= & \sum_{k \geq 0} \mathbf{E}\left[\eta_{i}^{+}(t)-\eta_{i}(t) \mid \eta_{0}(0)=k\right] F(k) \nu_{0}^{\rho}(k) \\
= & \mathbf{E}\left[\left(\eta_{i}^{+}(t)-\eta_{i}(t)\right) F\left(\eta_{0}(0)\right)\right] \\
= & \mathbf{E}\left[\mathbf{1}\{Q(t)=i\} F\left(\eta_{0}(0)\right)\right] \\
= & \sum_{k \geq 0} \mathbf{E}\left[\mathbf{1}\{Q(t)=i\} \mid \eta_{0}(0)=k\right] F(k) \nu_{0}^{\rho}(k) \\
= & \operatorname{Var}^{\rho}\left(\eta_{0}\right) \sum_{k \geq 0} \mathbf{E}\left[\mathbf{1}\{Q(t)=i\} \mid \eta_{0}(0)=k\right] \hat{\nu}_{0}^{\rho}(k) \\
= & \operatorname{Var}^{\rho}\left(\eta_{0}\right) \widehat{\mathbf{P}}^{\rho}\{Q(t)=i\} .
\end{aligned}
$$

This proves (6.6).

\subsection{Coupling for the zero range process}

Next we describe a coupling of two processes with labeled discrepancies (second class particles) between them, and then two randomly evolving labels that achieve simultaneously both goals (1) and (2) mentioned above. This construction works for any TAZRP with concave jump rate function $g$. Getting tail bounds for the label processes is the serious bottleneck of this proof, and that is where we need the restrictive assumption (6.9).

Let two processes $\eta \leq \omega$ evolve in basic coupling. This pair $(\eta, \omega)$ together with the labeled and ordered $\omega-\eta$ second class particles $\cdots \leq X_{-2}(t) \leq X_{-1}(t) \leq X_{0}(t) \leq X_{1}(t) \leq X_{2}(t) \leq \cdots$ form a "background" process on which we define two label processes $y(t)$ and $z(t)$. The $\omega-\eta$ second class particles are kept in order by requiring that, whenever a second class particle jumps to the right, the $X$-particle with highest label is moved.

The label processes will satisfy $y(t) \leq z(t)$, we will be able to bound $y(t)$ stochastically from above, $z(t)$ stochastically from below, and the following 
two pairs of processes will individually be in basic coupling:

$$
\left(\omega^{-}, \omega\right)=\left(\omega-\delta_{X_{y}}, \omega\right) \text { and }\left(\eta, \eta^{+}\right)=\left(\eta, \eta+\delta_{X_{z}}\right) .
$$

The definition of the label processes is partly forced on us by the requirement that jumps of $X_{y}$ and $X_{z}$ must replicate the rates required by the basic coupling. Additionally, we devise the joint process $(y, z)$ so that the order $y \leq z$ is maintained.

The rule is that after each jump among $(\omega, \eta)$ that in any way affects the site where $X_{y}$ resides, the value of $y$ is refreshed randomly. Let $a$ and $b$ denote the minimal and maximal labels at the site $i$ where $X_{y}$ resides after the jump. If $X_{y}$ resides at a site other than $X_{z}$, then $y$ chooses a new value $y^{\prime}$ according to these probabilities:

$$
y^{\prime}=\left\{\begin{array}{lll}
a & \text { with probability } & \frac{g\left(\omega_{i}-1\right)-g\left(\eta_{i}\right)}{g\left(\omega_{i}\right)-g\left(\eta_{i}\right)} \\
b & \text { with probability } & \frac{g\left(\omega_{i}\right)-g\left(\omega_{i}-1\right)}{g\left(\omega_{i}\right)-g\left(\eta_{i}\right)} .
\end{array}\right.
$$

If $g\left(\omega_{i}\right)-g\left(\eta_{i}\right)=0$ then $y^{\prime}=a$.

Similarly, after a jump in the background process that affects the site where $X_{z}$ resides, if $X_{z}$ and $X_{y}$ are not together, then $z$ takes the new value $z^{\prime}$ as follows (with $b$ again the maximal label at the site $i=X_{z}$ after the jump):

$$
z^{\prime}=\left\{\begin{array}{lll}
b-1 & \text { with probability } & \frac{g\left(\omega_{i}\right)-g\left(\eta_{i}+1\right)}{g\left(\omega_{i}\right)-g\left(\eta_{i}\right)} \\
b & \text { with probability } & \frac{g\left(\eta_{i}+1\right)-g\left(\eta_{i}\right)}{g\left(\omega_{i}\right)-g\left(\eta_{i}\right)}
\end{array}\right.
$$

If $g\left(\omega_{i}\right)-g\left(\eta_{i}\right)=0$ then $z^{\prime}=b$.

Finally, if after the jump $X_{y}=X_{z}=i$, then the labels are refreshed jointly as follows:

$$
\left(\begin{array}{l}
y^{\prime} \\
z^{\prime}
\end{array}\right)=\left\{\begin{array}{cc}
\left(\begin{array}{c}
a \\
b-1
\end{array}\right) & \text { with probability } \frac{g\left(\omega_{i}\right)-g\left(\eta_{i}+1\right)}{g\left(\omega_{i}\right)-g\left(\eta_{i}\right)} \\
\left(\begin{array}{l}
a \\
b
\end{array}\right) & \text { with probability } \frac{g\left(\eta_{i}+1\right)-g\left(\eta_{i}\right)}{g\left(\omega_{i}\right)-g\left(\eta_{i}\right)} \\
-\frac{g\left(\omega_{i}\right)-g\left(\omega_{i}-1\right)}{g\left(\omega_{i}\right)-g\left(\eta_{i}\right)} \\
\left(\begin{array}{l}
b \\
b
\end{array}\right) & \text { with probability } \frac{g\left(\omega_{i}\right)-g\left(\omega_{i}-1\right)}{g\left(\omega_{i}\right)-g\left(\eta_{i}\right)}
\end{array}\right.
$$


If $g\left(\omega_{i}\right)-g\left(\eta_{i}\right)=0$ then $\left(y^{\prime}, z^{\prime}\right)=(a, b)$.

The jump rules preserve $y \leq z$. Note that marginally $y^{\prime}$ obeys probabilities (6.12), and similarly for $z^{\prime}$. Concavity of $g$ was used to define the middle case in the joint rule.

Let us observe why these rules give the pair $\left(\omega^{-}, \omega\right)=\left(\omega-\delta_{X_{y}}, \omega\right)$ the same rates this pair would have in basic coupling. The requirement is that a jump across edge $(i, i+1)$ occur for both processes at rate $g\left(\omega_{i}^{-}\right)$, and only for $\omega$ at rate $g\left(\omega_{i}\right)-g\left(\omega_{i}^{-}\right)$. This requires thinking through a few cases. Only the site where $X_{y}$ resides needs attention since elsewhere $\left(\omega^{-}, \omega\right)$ jump together according to ZRP rates.

(i) In the basic coupling of $(\eta, \omega)$, an $(i, i+1)$ jump occurs in $\eta$ at rate $g\left(\eta_{i}\right)$. Then both $\omega$ and $\omega^{-}$experience this jump.

(ii) An $\omega-\eta$ second class particle jumps at rate $g\left(\omega_{i}\right)-g\left(\eta_{i}\right)$. Prior to this jump $y$ chose the top label with probability given by the second line of (6.12), hence the rate at which $X_{y}$ jumps is

$$
\left(g\left(\omega_{i}\right)-g\left(\eta_{i}\right)\right) \cdot \frac{g\left(\omega_{i}\right)-g\left(\omega_{i}-1\right)}{g\left(\omega_{i}\right)-g\left(\eta_{i}\right)}=g\left(\omega_{i}\right)-g\left(\omega_{i}-1\right) .
$$

Thus at this rate $\omega$ experiences the jump but $\omega^{-}$does not.

If prior to this jump $y$ chose the bottom label, then both $\omega$ and $\omega^{-}$ experience this jump, and this happens with rate

$$
\left(g\left(\omega_{i}\right)-g\left(\eta_{i}\right)\right) \cdot \frac{g\left(\omega_{i}-1\right)-g\left(\eta_{i}\right)}{g\left(\omega_{i}\right)-g\left(\eta_{i}\right)}=g\left(\omega_{i}-1\right)-g\left(\eta_{i}\right) .
$$

Adding up the rates we see that the rates of basic coupling have been realized. A similar argument is given for $\left(\eta, \eta^{+}\right)=\left(\eta, \eta+\delta_{X_{z}}\right)$.

We come to the unique point in the proof where assumption (6.9) is used, namely the tail bounds for the labels.

Lemma 6.1. Let the labels start with $y(0)=z(0)=0$. Under assumption (6.9) we have these bounds: $\mathbf{P}\{y(t) \geq k\} \leq r^{k}$ and $\mathbf{P}\{z(t) \leq-k\} \leq r^{k}$ for all $k \in \mathbb{Z}_{+}$and $t \geq 0$.

Proof. We do the proof for $y(t)$. The bounds are valid conditionally on the evolution $(\eta, \omega)$ of the background process. So assume this background evolution given. Then we think of $y(t)$ as an integer-valued Markov chain that is subject to jumps triggered by the background environment. Each jump happens at some site $i$ with range of labels $\{a, \ldots, b\}$ and occupation variables $\omega_{i}>\eta_{i} \geq 0$ that together satisfy

$$
\omega_{i}-\eta_{i}=b-a+1 .
$$


Given the current value $y$, the new value $y^{\prime}$ is obtained by the following rules, which of course are consistent with (6.12): if $g\left(\omega_{i}\right)-g\left(\eta_{i}\right)=0$ then

$$
y^{\prime}= \begin{cases}y & \text { if } y<a \text { or } y>b \\ a & \text { if } y \in\{a, \ldots, b\}\end{cases}
$$

while if $g\left(\omega_{i}\right)-g\left(\eta_{i}\right)>0$ then

$$
y^{\prime}=\left\{\begin{array}{lll}
y & \text { if } y<a \text { or } y>b \\
a & \text { with probability } & \frac{g\left(\omega_{i}-1\right)-g\left(\eta_{i}\right)}{g\left(\omega_{i}\right)-g\left(\eta_{i}\right)} \text { if } y \in\{a, \ldots, b\} \\
b & \text { with probability } & \frac{g\left(\omega_{i}\right)-g\left(\omega_{i}-1\right)}{g\left(\omega_{i}\right)-g\left(\eta_{i}\right)} \text { if } y \in\{a, \ldots, b\} .
\end{array}\right.
$$

There are infinitely many such jumps in any finite time interval, but all but finitely many leave $y$ unchanged. To get around this difficulty, we can first freeze all the Poisson clocks outside space interval $[-M, M]$, prove the lemma there, and then let $M \nearrow \infty$. Since rates are bounded, in any given bounded block of space-time the finite- $M$ process agrees with the infinite process for all large enough $M$.

To prove the lemma we show that every jump of type (6.13)-(6.14) preserves the geometric tail bound, regardless of the values $a, b, \omega_{i}, \eta_{i}$. So suppose $y$ is an integer-valued random variable such that

$$
P(y \geq k) \leq r^{k} \quad \text { for } \quad k \geq 0,
$$

and define $y^{\prime}$ via (6.13)-(6.14). We wish to show that $P\left(y^{\prime} \geq k\right) \leq r^{k}$ for $k \geq 0$.

The case $(6.13)$ is clear since there $y^{\prime} \leq y$. Let us consider the case $g\left(\omega_{i}\right)-g\left(\eta_{i}\right)>0$. Since the jump only redistributes the probability mass in $\{a, \ldots, b\}$ to $\{a, b\}$, it suffices to check that

$$
P\left(y^{\prime} \geq b\right) \leq r^{b}
$$

in the case $b \geq 0$. Using the jump rule (6.14),

$$
\begin{aligned}
P\left(y^{\prime} \geq b\right) & =P\left(y^{\prime}=b\right)+P\left(y^{\prime} \geq b+1\right) \\
& =\frac{g\left(\omega_{i}\right)-g\left(\omega_{i}-1\right)}{g\left(\omega_{i}\right)-g\left(\eta_{i}\right)} P(a \leq y \leq b)+P(y \geq b+1) .
\end{aligned}
$$

If $g\left(\omega_{i}\right)-g\left(\omega_{i}-1\right)=0$ the conclusion (6.15) follows from the assumption on $y$. So we assume $g\left(\omega_{i}\right)-g\left(\omega_{i}-1\right)>0$. Then by concavity all the 
$g$-increments between $\eta_{i}, \ldots, \omega_{i}$ are positive. Next write

$$
\begin{gathered}
P\left(y^{\prime} \geq b\right)=\frac{g\left(\omega_{i}\right)-g\left(\omega_{i}-1\right)}{g\left(\omega_{i}\right)-g\left(\eta_{i}\right)} \sum_{k=a}^{b}(1-r) r^{k} \\
+\frac{g\left(\omega_{i}\right)-g\left(\omega_{i}-1\right)}{g\left(\omega_{i}\right)-g\left(\eta_{i}\right)}\left(P(a \leq y \leq b)-r^{a}+r^{b+1}\right) \\
+P(y \geq b+1) .
\end{gathered}
$$

For $a \leq k \leq b$

$$
\begin{aligned}
(1-r) r^{k} & =(1-r) r^{b} \cdot \frac{1}{r^{b-k}} \leq(1-r) r^{b} \prod_{\ell=\omega_{i}-b+k}^{\omega_{i}-1} \frac{g(\ell)-g(\ell-1)}{g(\ell+1)-g(\ell)} \\
& =(1-r) r^{b} \cdot \frac{g\left(\omega_{i}-b+k\right)-g\left(\omega_{i}-b+k-1\right)}{g\left(\omega_{i}\right)-g\left(\omega_{i}-1\right)} .
\end{aligned}
$$

Adding these up over $a \leq k \leq b$ gives

first term on the right in (6.16)

$$
\begin{aligned}
& \leq \frac{g\left(\omega_{i}\right)-g\left(\omega_{i}-1\right)}{g\left(\omega_{i}\right)-g\left(\eta_{i}\right)}(1-r) r^{b} \\
& \quad \times \sum_{k=a}^{b} \frac{g\left(\omega_{i}-b+k\right)-g\left(\omega_{i}-b+k-1\right)}{g\left(\omega_{i}\right)-g\left(\omega_{i}-1\right)} \\
& =(1-r) r^{b} \cdot \frac{g\left(\omega_{i}\right)-g\left(\omega_{i}-b+a-1\right)}{g\left(\omega_{i}\right)-g\left(\eta_{i}\right)}=(1-r) r^{b}=r^{b}-r^{b+1} .
\end{aligned}
$$

Substitute this bound back up to (6.16) and use $P(y \geq k) \leq r^{k}$ twice:

$$
\begin{aligned}
P\left(y^{\prime} \geq b\right) \leq & r^{b}+P(y \geq b+1)-r^{b+1} \\
& \quad+\frac{g\left(\omega_{i}\right)-g\left(\omega_{i}-1\right)}{g\left(\omega_{i}\right)-g\left(\eta_{i}\right)}\left(P(a \leq y \leq b)-r^{a}+r^{b+1}\right) \\
& \leq r^{b}+\frac{g\left(\omega_{i}\right)-g\left(\omega_{i}-1\right)}{g\left(\omega_{i}\right)-g\left(\eta_{i}\right)}\left(P(y \geq a)-r^{a}\right) \\
& \leq r^{b} .
\end{aligned}
$$

Thus (6.15) has been checked and thereby the lemma has been proved for $y(t)$.

\section{References}

The results for ZRP are proved in [BKS08]. The case of constant jump rate was done earlier in [BK08]. 



\section{Bibliography}

[BAC09] Gérard Ben Arous and Ivan Corwin, Current fluctuations for TASEP: a proof of the Prähofer-Spohn conjecture, arXiv:0905.2993 (2009).

[Bal03] Márton Balázs, Growth fluctuations in a class of deposition models, Ann. Inst. H. Poincaré Probab. Statist. 39 (2003), no. 4, 639-685. MR MR1983174 (2005j:60178a)

[BCS06] Márton Balázs, Eric Cator, and Timo Seppäläinen, Cube root fluctuations for the corner growth model associated to the exclusion process, Electron. J. Probab. 11 (2006), no. 42, 1094-1132 (electronic). MR MR2268539

[BFP] Jinho Baik, Patrik L. Ferrari, and Sandrine Péché, Limit process of stationary tasep near the characteristic line, arXiv:0907.0226.

[BG97] Lorenzo Bertini and Giambattista Giacomin, Stochastic Burgers and KPZ equations from particle systems, Comm. Math. Phys. 183 (1997), no. 3, 571-607. MR MR1462228 (99e:60212)

[BK08] Márton Balázs and Júlia Komjáthy, Order of current variance and diffusivity in the rate one totally asymmetric zero range process, J. Stat. Phys. 133 (2008), no. 1, 59-78. MR MR2438897 (2009h:60157)

[BKS08] Márton Balázs, Júlia Komjáthy, and Timo Seppäläinen, Microscopic concavity and fluctuation bounds in a class of deposition processes, arXiv:0808.1177 (2008).

[BQS] Márton Balázs, Jeremy Quastel, and Timo Seppäläinen, Scaling exponent for the Hopf-Cole solution of KPZ/stochastic Burgers, arXiv:0909.4816. 
[BRAS06] Márton Balázs, Firas Rassoul-Agha, and Timo Seppäläinen, The random average process and random walk in a space-time random environment in one dimension, Comm. Math. Phys. 266 (2006), 499-545.

[BS] Albert-László Barabási and H. Eugene Stanley, Fractal concepts in surface growth. Cambridge University Press, 1995.

[BS07] Márton Balázs and Timo Seppäläinen, Exact connections between current fluctuations and the second class particle in a class of deposition models, J. Statist. Phys. 127 (2007), 431455 .

[BS09a] Márton Balázs and Timo Seppäläinen, Fluctuation bounds for the asymmetric simple exclusion process, ALEA Lat. Am. J. Probab. Math. Stat. 6 (2009), 1-24. MR MR2485877

[BS09b] Márton Balázs and Timo Seppäläinen, Order of current variance and diffusivity in the asymmetric simple exclusion process, arXiv:math/0608400, to appear in Ann. of Math.

[BW71] P. J. Bickel and M. J. Wichura, Convergence criteria for multiparameter stochastic processes and some applications, Ann. Math. Statist. 42 (1971), 1656-1670. MR MR0383482 (52 \#4363)

[CFP] Ivan Corwin, Patrik L. Ferrari, and Sandrine Péché, Limit processes of non-equilibrium tasep, arXiv:1002.3476.

[CG06] Eric Cator and Piet Groeneboom, Second class particles and cube root asymptotics for Hammersley's process, Ann. Probab. 34 (2006), no. 4, 1273-1295. MR MR2257647 (2008e:60021)

[DGL85] D. Dürr, S. Goldstein, and J. Lebowitz, Asymptotics of particle trajectories in infinite one-dimensional systems with collisions, Comm. Pure Appl. Math. 38 (1985), no. 5, 573-597. MR MR803248 (87e:60166)

[Dur04] Richard Durrett, Probability: theory and examples, third ed., Duxbury Advanced Series, Brooks/Cole-Thomson, Belmont, CA, 2004.

[FF94] P. A. Ferrari and L. R. G. Fontes, Current fluctuations for the asymmetric simple exclusion process, Ann. Probab. 22 (1994), no. 2, 820-832. MR 95j:60162

[FF98] _ Fluctuations of a surface submitted to a random average process, Electron. J. Probab. 3 (1998), no. 6, 34 pp. (electronic). MR 99e:60214 
[FMV03] L. R. G. Fontes, D. P. Medeiros, and M. Vachkovskaia, Time fluctuations of the random average process, Stochastic Process. Appl. 103 (2003), no. 2, 257-276. MR MR1950766 (2003k:60255)

[FS06] Patrik L. Ferrari and Herbert Spohn, Scaling limit for the spacetime covariance of the stationary totally asymmetric simple exclusion process, Comm. Math. Phys. 265 (2006), no. 1, 1-44. MR MR2217295 (2007g:82038a)

[Joh00] Kurt Johansson, Shape fluctuations and random matrices, Comm. Math. Phys. 209 (2000), no. 2, 437-476. MR 2001h:60177

[KKS75] H. Kesten, M. V. Kozlov, and F. Spitzer, A limit law for random walk in a random environment, Compositio Math. 30 (1975), 145-168. MR MR0380998 (52 \#1895)

[Kum08] Rohini Kumar, Space-time current process for independent random walks in one dimension, ALEA Lat. Am. J. Probab. Math. Stat. 4 (2008), 307-336. MR MR2456971

[Lig85] Thomas M. Liggett, Interacting particle systems, Grundlehren der Mathematischen Wissenschaften [Fundamental Principles of Mathematical Sciences], vol. 276, Springer-Verlag, New York, 1985. MR 86e:60089

[Pet08] Jonathon Peterson, Limiting distributions and large deviations for random walks in random environments, Ph.D. thesis, University of Minnesota, arXiv:0810.0257, 2008.

[PS] Jonathon Peterson and Timo Seppäläinen, Current fluctuations of a system of one-dimensional random walks in random environment, arXiv:0904.4768, to appear in Ann. Probab.

[PS08] Magda Peligrad and Sunder Sethuraman, On fractional Brownian motion limits in one dimensional nearest-neighbor symmetric simple exclusion, ALEA Lat. Am. J. Probab. Math. Stat. 4 (2008), 245-255. MR MR2448774

[PZ09] Jonathon Peterson and Ofer Zeitouni, Quenched limits for transient, zero-speed one-dimensional random walk in random environment, Ann. Probab. 37 (2009), no. 1, 143-188.

[QV07] J. Quastel and B. Valkó, $t^{1 / 3}$ Superdiffusivity of finite-range asymmetric exclusion processes on $\mathbb{Z}$, Comm. Math. Phys. 273 (2007), no. 2, 379-394. MR MR2318311 
[QV08] J. Quastel and B. Valkó, A note on the diffusivity of finite-range asymmetric exclusion processes on $\mathbb{Z}$, In and out equilibrium 2 , Progress in Probability (V. Sidoravicius and M. E. Vares, eds.), vol. 60 , Birkhäuser, 2008, pp. 543-550.

[RAS05] Firas Rassoul-Agha and Timo Seppäläinen, An almost sure invariance principle for random walks in a space-time random environment, Probab. Theory Related Fields 133 (2005), no. 3, 299-314. MR MR2198014

[Sep05] Timo Seppäläinen, Second-order fluctuations and current across characteristic for a one-dimensional growth model of independent random walks, Ann. Probab. 33 (2005), no. 2, 759 797. MR MR2123209 (2006d:60153)

[Sep08]_ Directed random growth models on the plane, Analysis and Stochastics of Growth Processes and Interface Models (P. Mörters et al., eds.), Oxford University Press, Oxford, UK, 2008, pp. 9-38.

[Set03] Sunder Sethuraman, An equivalence of $H_{-1}$ norms for the simple exclusion process, Ann. Probab. 31 (2003), no. 1, 35-62. MR MR1959785 (2003k:60264)

[Sol75] Fred Solomon, Random walks in a random environment, Ann. Probability 3 (1975), 1-31. MR MR0362503 (50 \#14943)

[Spi76] Frank Spitzer, Principles of random walks, second ed., SpringerVerlag, New York, 1976, Graduate Texts in Mathematics, Vol. 34. MR MR0388547 (52 \#9383)

[TW08a] Craig A. Tracy and Harold Widom, A Fredholm determinant representation in ASEP, J. Stat. Phys. 132 (2008), no. 2, 291300. MR MR2415104 (2009d:82108)

[TW08b] - Integral formulas for the asymmetric simple exclusion process, Comm. Math. Phys. 279 (2008), no. 3, 815-844. MR MR2386729 (2009e:60217)

[TW09a] - Asymptotics in ASEP with step initial condition, Comm. Math. Phys. 290 (2009), no. 1, 129-154. MR MR2520510

[TW09b] - Total current fluctuations in the asymmetric simple exclusion process, J. Math. Phys. 50 (2009), no. 9, 095204, 4. MR MR2566884 
[TW10] _ Formulas for joint probabilities for the asymmetric simple exclusion process, arXiv:1003.3431 (2010).

[Wal86] John B. Walsh, An introduction to stochastic partial differential equations, École d'été de probabilités de Saint-Flour, XIV1984, Lecture Notes in Math., vol. 1180, Springer, Berlin, 1986, pp. 265-439. MR MR876085 (88a:60114)

[Zei04] Ofer Zeitouni, Random walks in random environments, Lecture Notes in Mathematics, vol. 1837, Springer-Verlag, Berlin, 2004, Lectures from the 31st Summer School on Probability Theory held in Saint-Flour, July 8-25, 2001, Edited by Jean Picard. MR MR2071629

Timo Seppäläinen

Mathematics Department, University of Wisconsin-Madison

Madison, Wisconsin 53706, USA

seppalai@math.wisc.edu

http://www.math.wisc.edu/ seppalai 